\title{
A Survey on Wireless Security: Technical Challenges, Recent Advances, and Future Trends
}

\author{
By Yulong Zou, Senior Member IEEe, Jia Zhu, Xianbin Wang, Senior Member IEEE, And \\ Lajos Hanzo, Fellow IEEE
}

ABSTRACT | Due to the broadcast nature of radio propagation, the wireless air interface is open and accessible to both authorized and illegitimate users. This completely differs from a wired network, where communicating devices are physically connected through cables and a node without direct association is unable to access the network for illicit activities. The open communications environment makes wireless transmissions more vulnerable than wired communications to malicious attacks, including both the passive eavesdropping for data interception and the active jamming for disrupting legitimate transmissions. Therefore, this paper is motivated to examine the security vulnerabilities and threats imposed by the inherent open nature of wireless communications and to devise efficient defense mechanisms for improving the wireless network security. We first summarize the security requirements of wireless networks, including their authenticity, confidentiality, integrity, and availability issues. Next, a comprehensive overview of security attacks encountered in wireless networks is presented in view of the network protocol architecture, where the potential security threats are discussed at each protocol layer. We also provide

Manuscript received May 30, 2014; revised October 6, 2014; accepted April 21, 2016 This work was supported in part by the Priority Academic Program Development of Jiangsu Higher Education Institutions; the National Natural Science Foundation of China under Grants 61302104, 61401223, and 61522109; the Natural Science

Foundation of Jiangsu Province under Grants BK20140887 and BK20150040; and the Key Project of Natural Science Research of Higher Education Institutions of Jiangsu Province under Grant 15KJA510003.

Y. Zou and J. Zhu are with the School of Telecommunications and Information

Engineering, Nanjing University of Posts and Telecommunications, Nanjing 210003,

China (e-mail: yulong.zou@njupt.edu.cn; jiazhu@njupt.edu.cn).

X. Wang is with the Electrical and Computer Engineering Department, University of

Western Ontario, London, ON N6A 3K7, Canada (e-mail: xianbin.wang@uwo.ca).

L. Hanzo is with the School of Electronics and Computer Science, University of

Southampton, Southampton SO17 1BJ, U.K. (e-mail: Ih@ecs.soton.ac.uk).

Digital Object Identifier: 10.1109/JPROC.2016.255852 a survey of the existing security protocols and algorithms that are adopted in the existing wireless network standards, such as the Bluetooth, Wi-Fi, WiMAX, and the long-term evolution (LTE) systems. Then, we discuss the state of the art in physical-layer security, which is an emerging technique of securing the open communications environment against eavesdropping attacks at the physical layer. Several physical-layer security techniques are reviewed and compared, including information-theoretic security, artificial-noise-aided security, security-oriented beamforming, diversity-assisted security, and physical-layer key generation approaches. Since a jammer emitting radio signals can readily interfere with the legitimate wireless users, we also introduce the family of various jamming attacks and their countermeasures, including the constant jammer, intermittent jammer, reactive jammer, adaptive jammer, and intelligent jammer. Additionally, we discuss the integration of physical-layer security into existing authentication and cryptography mechanisms for further securing wireless networks. Finally, some technical challenges which remain unresolved at the time of writing are summarized and the future trends in wireless security are discussed.

KEYWORDS | Artificial noise; beamforming; denial of service (DoS); diversity; eavesdropping attack; information-theoretic security; jamming; network protocol; wireless jamming; wireless networks; wireless security

\section{NOMENCLATURE}

3G Third generation.

AAA Authentication, authorization, and accounting.

ACK Acknowledgement. 


\begin{tabular}{|c|c|}
\hline AES & Advanced encryption standard. \\
\hline AKA & Authentication and key agreement. \\
\hline AoA & Angle of arrival. \\
\hline AP & Access point. \\
\hline ARQ & Automatic Repeat reQuest. \\
\hline ASK & Authenticated secret key. \\
\hline BS & Base station. \\
\hline CDMA & Code division multiple access. \\
\hline $\mathrm{CK}(\mathrm{s})$ & Ciphering key(s). \\
\hline CSI & Channel state information. \\
\hline CSMA/CA & $\begin{array}{l}\text { Carrier sense multiple access with } \\
\text { collision avoidance. }\end{array}$ \\
\hline CST & Carrier sensing time. \\
\hline CTS & Clear to send. \\
\hline DA & Destination address. \\
\hline DCF & Distributed coordination function. \\
\hline DES & Data encryption standard. \\
\hline DIFS & Distributed interframe space. \\
\hline DN & Destination node. \\
\hline DSSS & Direct-sequence spread spectrum. \\
\hline DoS & Denial of service. \\
\hline EPC & Evolved packet core. \\
\hline E-UTRAN & $\begin{array}{l}\text { Evolved-universal terrestrial radio } \\
\text { access network. }\end{array}$ \\
\hline FFT & Fast Fourier transform. \\
\hline FHSS & Frequency-hopping spread spectrum. \\
\hline FTP & File transfer protocol. \\
\hline GSVD & Generalized singular value decomposition. \\
\hline HSS & Home subscriber server. \\
\hline HTTP & Hypertext transfer protocol. \\
\hline ICMP & Internet control message protocol. \\
\hline $\mathrm{ICV}$ & Integrity check value. \\
\hline $\mathrm{IK}(\mathrm{s})$ & Integrity key(s). \\
\hline IMSI & International mobile subscriber identity. \\
\hline IP & Internet protocol. \\
\hline IV & Initialization vector. \\
\hline LTE & Long-term evolution. \\
\hline MAC & Medium-access control. \\
\hline MIC & Message integrity check. \\
\hline MIMO & Multiple-input-multiple-output. \\
\hline MISOME & $\begin{array}{l}\text { Multiple-input-single-output } \\
\text { multiple eavesdropper. }\end{array}$ \\
\hline MITM & Man in the middle. \\
\hline MME & Mobility management entity. \\
\hline NIC & Network interface controller. \\
\hline NP & Nondeterministic polynomial. \\
\hline OFDMA & $\begin{array}{l}\text { Orthogonal frequency-division } \\
\text { multiple access. }\end{array}$ \\
\hline OSI & Open systems interconnection. \\
\hline PER & Packet error rate. \\
\hline PKM & Privacy and key management. \\
\hline $\mathrm{PN}$ & Pseudonoise. \\
\hline PRNG & Pseudorandom number generator. \\
\hline QoS & Quality of service. \\
\hline RFCOMM & Radio-frequency communications. \\
\hline RSA & Rivest-Shamir-Adleman. \\
\hline
\end{tabular}

$\begin{array}{ll}\text { RSS } & \text { Received signal strength. } \\ \text { RTS } & \text { Request to send. } \\ \text { SA } & \text { Source address. } \\ \text { SIFS } & \text { Short interframe space. } \\ \text { SINR } & \text { Signal-to-interference-and-noise ratio. } \\ \text { SQL } & \text { Structured query language. } \\ \text { SMTP } & \text { Simple mail transfer protocol. } \\ \text { SN } & \text { Source node. } \\ \text { SNR } & \text { Signal-to-noise ratio. } \\ \text { SS } & \text { Subscriber station. } \\ \text { SSL } & \text { Secure sockets layer. } \\ \text { TA } & \text { Transmitter address. } \\ \text { TCP } & \text { Transmission control protocol. } \\ \text { TDMA } & \text { Time-division multiple access. } \\ \text { TK } & \text { Temporal key. } \\ \text { TKIP } & \text { Temporal key integrity protocol. } \\ \text { TLS } & \text { Transport layer security. } \\ \text { TSC } & \text { TKIP sequence counter. } \\ \text { TTAK } & \text { TKIP-mixed transmit address and key. } \\ \text { TTLS } & \text { Tunneled transport layer security. } \\ \text { UDP } & \text { User datagram protocol. } \\ \text { UE } & \text { User equipment. } \\ \text { UMTS } & \text { Universal mobile } \\ \text { WEP } & \text { telecommunications system. } \\ \text { WiMAX } & \text { Worldwide interoperability for } \\ \text { WLAN } & \text { microwave access. } \\ \text { WMAN } & \text { Wireless metropolitan area network. } \\ \text { WPA } & \text { Wi-Fi protected access. } \\ \text { WPA2 } & \text { Wi-Fi protected access II. } \\ \text { WPAN } & \text { Wireless personal area network. } \\ & \end{array}$

\section{INTRODUCTION}

During the past decades, wireless communications infrastructure and services have been proliferating with the goal of meeting rapidly increasing demands [1], [2]. According to the latest statistics released by the International Telecommunications Union in 2013 [3], the number of mobile subscribers has reached 6.8 billion worldwide and almost $40 \%$ of the world's population is now using the Internet. Meanwhile, it has been reported in [4] that an increasing number of wireless devices are abused for illicit cybercriminal activities, including malicious attacks, computer hacking, data forging, financial information theft, online bullying/stalking, and so on. This causes the direct loss of about 83 billion euros with an estimated 556 million users worldwide impacted by cybercrime each year, according to the 2012 Norton cybercrime report [4]. Hence, it is of paramount importance to improve wireless communications security to fight against cybercriminal activities, especially because more and more people are using wireless networks (e.g., cellular networks and Wi-Fi) for online banking and 
personal e-mails, owing to the widespread use of smartphones.

Wireless networks generally adopt the OSI protocol architecture [5] comprising the application layer, transport layer, network layer [6], MAC layer [7] and physical layer [8], [9]. Security threats and vulnerabilities associated with these protocol layers are typically protected separately at each layer to meet the security requirements, including the authenticity, confidentiality, integrity and availability [10]. For example, cryptography is widely used for protecting the confidentiality of data transmission by preventing information disclosure to unauthorized users [11], [12]. Although cryptography improves the achievable communications confidentiality, it requires additional computational power and imposes latency [13], since a certain amount of time is required for both data encryption and decryption [14]. In order to guarantee the authenticity of a caller or receiver, existing wireless networks typically employ multiple authentication approaches simultaneously at different protocol layers, including MAC-layer authentication [15], networklayer authentication [16], [17], and transport-layer authentication [18]. To be specific, in the MAC layer, the MAC address of a user should be authenticated to prevent unauthorized access. In the network layer, the WPA and the WPA2 are two commonly used network-layer authentication protocols [19], [20]. Additionally, the transport-layer authentication includes the SSL and its successor, namely the TLS protocols [21]-[23]. It becomes obvious that exploiting multiple authentication mechanisms at different protocol layers is capable of enhancing the wireless security, again, at the cost of high computational complexity and latency. As shown in Fig. 1, the main wireless security methodologies include the authentication, authorization and encryption, for which the diverse design factors, e.g., the security level, implementation complexity, and communication latency need to be balanced.

In wired networks, the communicating nodes are physically connected through cables. By contrast, wireless networks are extremely vulnerable owing to the

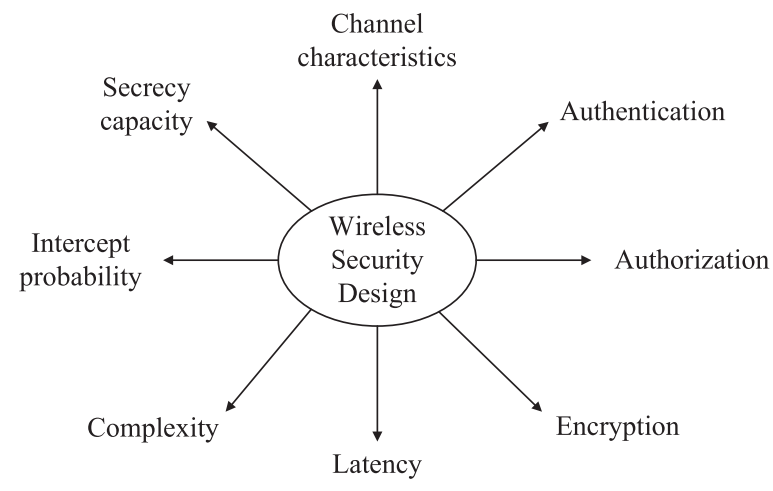

Fig. 1. Wireless security methodologies and design factors. broadcast nature of the wireless medium. Explicitly, wireless networks are prone to malicious attacks, including eavesdropping attack [24], DoS attack [25], spoofing attack [26], MITM attack [27], message falsification/ injection attack [28], etc. For example, an unauthorized node in a wireless network is capable of inflicting intentional interferences with the objective of disrupting data communications between legitimate users. Furthermore, wireless communications sessions may be readily overheard by an eavesdropper, as long as the eavesdropper is within the transmit coverage area of the transmitting node. In order to maintain confidential transmission, existing systems typically employ cryptographic techniques for preventing eavesdroppers from intercepting data transmissions between legitimate users [29], [30]. Cryptographic techniques assume that the eavesdropper has limited computing power and rely upon the computational hardness of their underlying mathematical problems. The security of a cryptographic approach would be compromised, if an efficient method of solving its underlying hard mathematical problem was to be discovered [31], [32].

Recently, physical-layer security is emerging as a promising means of protecting wireless communications to achieve information-theoretic security against eavesdropping attacks. In [33], Wyner examined a discrete memoryless wiretap channel consisting of a source, a destination as well as an eavesdropper and proved that perfectly secure transmission can be achieved, provided that the channel capacity of the main link from the source to the destination is higher than that of the wiretap link from the source to the eavesdropper. In [34], Wyner's results were extended from the discrete memoryless wiretap channel to the Gaussian wiretap channel, where the notion of a so-called secrecy capacity was developed, which was shown to be equal to the difference between the channel capacity of the main link and that of the wiretap link. If the secrecy capacity falls below zero, the transmissions from the source to the destination become insecure and the eavesdropper would become capable of intercepting the source's transmissions [35], [36]. In order to improve the attainable transmission security, it is of importance to increase the secrecy capacity by exploiting sophisticated signal processing techniques, such as the artificial-noise-aided security [37]-[39], securityoriented beamforming [40], [41], security-oriented diversity approaches [42], [43] and so on.

In this paper, we are motivated to discuss diverse wireless attacks as well as the corresponding defense mechanisms and to explore a range of challenging open issues in wireless security research. The main contributions of this paper are summarized as follows. First, a systematic review of security threats and vulnerabilities is presented at the different protocol layers, commencing from the physical layer up to the application layer. Second, we summarize the family of security protocols and 
algorithms used in the existing wireless networks, such as the Bluetooth, Wi-Fi, WiMAX and LTE standards. Third, we discuss the emerging physical-layer security in wireless communications and highlight the class of information-theoretic security, artificial-noise-aided security, security-oriented beamforming, security-oriented diversity, and physical-layer secret key generation techniques. Additionally, we provide a review on various wireless jammers (i.e., the constant jammer, intermittent jammer, reactive jammer, adaptive jammer, and intelligent jammer) as well as their detection and prevention techniques. Finally, we outline some of open challenges in wireless security.

The remainder of this paper is organized as follows. Section II presents the security requirements of wireless networks, where the authenticity, confidentiality, integrity, and availability of wireless services are discussed. In Section III, we analyze the security vulnerabilities and weaknesses of wireless networks at different protocol layers, including the application layer, the transport layer, the network layer, the MAC layer, and the physical layer. Next, in Section IV, the security protocols and algorithms used in existing wireless networks, such as the Bluetooth, Wi-Fi, WiMAX, and LTE standards, are discussed. Then, Section V presents the physical-layer security which is emerging as an effective paradigm conceived for improving the security of wireless communications against eavesdropping attacks by exploiting the physicallayer characteristics of wireless channels. In Section VI, we characterize the family of wireless jamming attacks and their countermeasures, while in Section VII, we discuss how physical-layer security may be invoked for efficiently complementing the existing suite of classic authentication and cryptography mechanisms. These discussions are followed by Section VIII, where some of the open challenges and future trends in wireless security are presented. Finally, Section IX provides our concluding remarks.

\section{SECURITY REQUIREMENTS IN WIRELESS NETWORKS}

Again, in wireless networks, the information is exchanged among authorized users, but this process is vulnerable to various malicious threats owing to the broadcast nature of the wireless medium. The security requirements of wireless networks are specified for the sake of protecting the wireless transmissions against wireless attacks, such as eavesdropping attack, DoS attack, data falsification attack, node compromise attack, and so on [44], [45]. For example, maintaining data confidentiality is a typical security requirement, which refers to the capability of restricting data access to authorized users only, while preventing eavesdroppers from intercepting the information. Generally speaking, secure wireless communications should satisfy the requirements of authenticity, confidentiality, integrity, and availability [46], as detailed in the following.

- Authenticity: Authenticity refers to confirming the true identity of a network node to distinguish authorized users from unauthorized users. In wireless networks, a pair of communicating nodes should first perform mutual authentication before establishing a communications link for data transmission [47]. Typically, a network node is equipped with a wireless network interface card and has a unique MAC address, which can be used for authentication purposes. Again, in addition to MAC authentication, there are other wireless authentication methods, including network-layer authentication, transport-layer authentication, and application-layer authentication.

- Confidentiality: The confidentiality refers to limiting the data access to intended users only, while preventing the disclosure of the information to unauthorized entities [48]. Considering the symmetric key encryption technique as an example, the source node first encrypts the original data (often termed as plaintext) using an encryption algorithm with the aid of a secret key that is shared with the intended destination only. Next, the encrypted plaintext (referred to as cipher text) is transmitted to the destination that then decrypts its received cipher text using the secret key. Since the eavesdropper has no knowledge of the secret key, it is unable to interpret the plaintext based on the overheard cipher text. Traditionally, the classic Diffie-Hellman key agreement protocol is used to achieve the key exchange between the source and destination and requires a trusted key management center [32]. Recently, physical-layer security has emerged as a means of protecting the confidentiality of wireless transmission against eavesdropping attacks for achieving information-theoretic security [33], [49]. The details of physical-layer security will be discussed in Section V.

- Integrity: The integrity of information transmitted in a wireless network should be accurate and reliable during its entire life-cycle representing the source information without any falsification and modification by unauthorized users. The data integrity may be violated by so-called insider attacks, such as, for example, node compromise attacks [50]-[52]. More specifically, a legitimate node that is altered and compromised by an adversary is termed as a compromised node. The compromised node may inflict damage upon the data integrity by launching malicious attacks, including message injection, false reporting, data modification, and so on. In general, it is quiet 
challenging to detect the attacks by compromised nodes, since these compromised nodes running malicious codes still have valid identities. A promising solution to detect compromised nodes is to utilize the automatic code update and recovery process, which guarantees that the nodes are periodically patched and a compromised node may be detected, if the patch fails. The compromised nodes can be repaired and revoked through the so-called code recovery process.

- Availability: The availability implies that the authorized users are indeed capable of accessing a wireless network anytime and anywhere upon request. The violation of availability, referred to as denial of service, will result in the authorized users to become unable to access the wireless network, which in turn results in unsatisfactory user experience [53], [54]. For example, any unauthorized node is capable of launching DoS activities at the physical layer by maliciously generating interferences for disrupting the desired communications between legitimate users, which is also known as a jamming attack. In order to combat jamming attacks, existing wireless systems typically consider the employment of spread spectrum techniques, including DSSS [55], [56] and FHSS solutions [57]. To be specific, DSSS employs a PN sequence to spread the spectrum of the original signal to a wide frequency bandwidth. In this way, the jamming attack operating without the knowledge of the PN sequence has to dissipate a much higher power for disrupting the legitimate transmission, which may not be feasible in practice due to its realistic power constraint. As an alternative, FHSS continuously changes the central frequency of the transmitted waveform using a certain frequency-hopping pattern, so that the jamming attacker cannot monitor and interrupt the legitimate transmissions.

The aforementioned authenticity, confidentiality, integrity, and availability are summarized in Table 1, which are commonly considered and implemented in the existing wireless networks, including the Bluetooth [58], Wi-Fi [59], WiMAX [60], LTE [61] standards, and so on. In principle, wireless networks should be as secure as wired networks. This implies that the security requirements of wireless networks should be the same as those of wired networks, including the requirements of authenticity, confidentiality, integrity, and availability. However, due to the broadcast nature of radio propagation, achieving these security requirements in wireless networks is more challenging than in wired networks. For example, the availability of wireless networks is extremely vulnerable, since a jamming attack imposing a radio signal can readily disrupt and block
Table 1 Summarization of Wireless Security Requirements

\begin{tabular}{|c|l|}
\hline Security Requirements & \multicolumn{1}{|c|}{ Specific Objectives to be Achieved } \\
\hline Authenticity & $\begin{array}{l}\text { Specified to differentiate authorized users from } \\
\text { unauthorized users }\end{array}$ \\
\hline Confidentiality & $\begin{array}{l}\text { Specified to limit the confidential data access to } \\
\text { intended users only }\end{array}$ \\
\hline Integrity & $\begin{array}{l}\text { Specified to guarantee the accuracy of the trans- } \\
\text { mitted information without any falsification }\end{array}$ \\
\hline Availability & $\begin{array}{l}\text { Specified to make sure that the authorized users } \\
\text { can access wireless network resources anytime } \\
\text { and anywhere upon request }\end{array}$ \\
\hline
\end{tabular}

the wireless physical-layer communications. Hence, compared to wired networks, wireless systems typically employ an additional DSSS (or FHSS) technique in order to protect the wireless transmissions against jamming attacks.

\section{SECURITY VULNERABILITIES IN WIRELESS NETWORKS}

In this section, we present a systematic review of various security vulnerabilities and weaknesses encountered in wireless networks. Apart from their differences, wired and wireless networks also share some similarities. For example, they both adopt the OSI layered protocol architecture consisting of the physical layer, the MAC layer, the network layer, the transport layer, and the application layer. As shown in Fig. 2, a network node (denoted by node A) employs these protocols for transmitting its

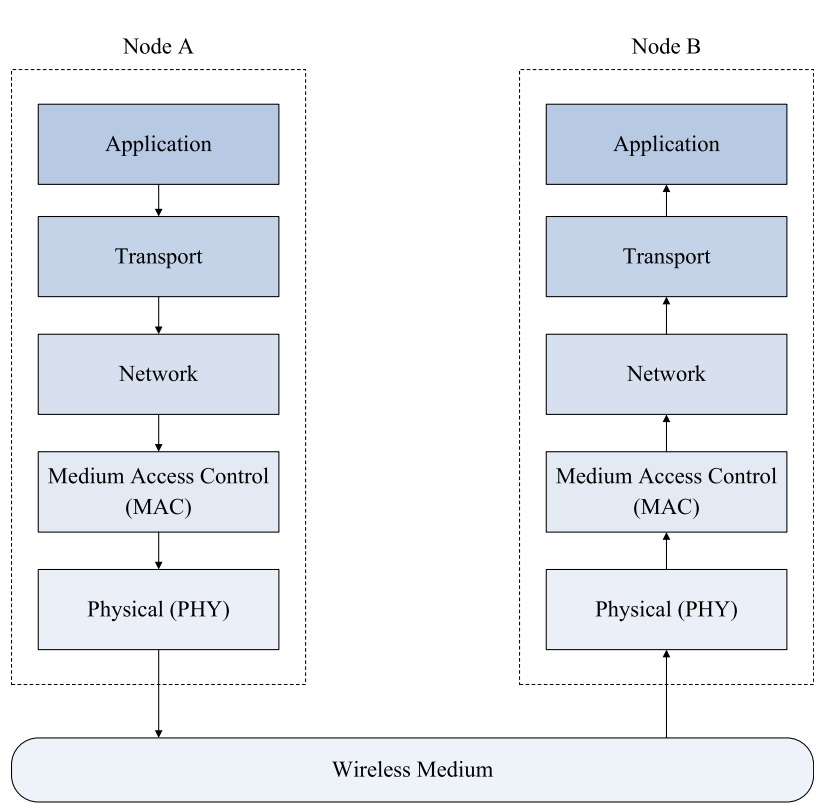

Fig. 2. Generic wireless osı layered protocol architecture consisting of the application layer, the transport layer, the network layer, the MAC layer, and the physical layer. 


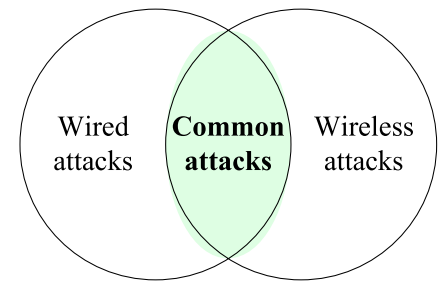

Fig. 3. Relationship between the wired and wireless attacks.

data packets to another network node (i.e., node B). To be specific, the data packet at node $\mathrm{A}$ is first extended with the protocol overheads, including the applicationlayer overhead, transport-layer overhead, network-layer overhead, MAC overhead, and physical-layer overhead. This results in an encapsulated packet. Then, the resultant data packet is transmitted via the wireless medium to node $\mathrm{B}$, which will perform packet decapsulation, commencing from the physical layer and proceeding upward to the application layer, in order to recover the original data packet. Note that the difference between the wired and wireless networks mainly lies in the PHY and MAC layers, while the application, transport, and network layers of wireless networks are typically identical to those of wired networks. As a consequence, the wired and wireless networks share some common security vulnerabilities owing to their identical application, transport, and network layers. Nevertheless, they also suffer from mutually exclusive attacks due to the fact that the wired and wireless networks have different PHY and MAC layers, as shown in Fig. 3.

Table 2 shows the main protocols and specifications implemented at each of wireless OSI layers. For example, the application-layer supports the HTTP for the sake of delivering web services, while the FTP is used for large file transfer, and the SMTP is invoked for electronic mail (e-mail) transmission and so on [62]. The commonly used transport-layer protocols include the TCP and the UDP [63], [64]. The TCP ensures the reliable and ordered delivery of data packets, whereas the UDP

Table 2 Main Protocols and Specifications of the Wireless OSI Layers

\begin{tabular}{|c|c|}
\hline OSI Layers & Main Protocols and Specifications \\
\hline Application & HTTP, FTP, SMTP [62] \\
\hline Transport & TCP, UDP [63], [64] \\
\hline Network & IP, ICMP [65] \\
\hline MAC & CSMA/CA, ALOHA, CDMA [66], OFDMA [67] \\
\hline PHY & Transmission Medium, Coding and Modulation \\
\hline
\end{tabular}

has no guarantee of such reliable and ordered delivery. In contrast to TCP, UDP has no handshaking dialogs and adopts a simpler transmission model, hence imposing a reduced protocol overhead. In the network layer, we also have different protocols, such as the IP, which was conceived for delivering data packets based on IP addresses, and the ICMP designed for sending error messages for indicating, for example, that a requested service is unavailable or that a network node could not be reached [65]. Regarding the MAC layer, there are numerous different protocols adopted by various wireless networks, such as the CSMA/CA used in Wi-Fi networks, the slotted ALOHA employed in tactical satellite networks by military forces, CDMA involved in 3G mobile networks [66] and OFDMA adopted in the LTE and LTE-advanced networks [67]. Additionally, the physical layer specifies the physical characteristics of information transmission, including the transmission medium, modulation, line coding, multiplexing, circuit switching, pulse shaping, forward error correction, bit interleaving, and other channel coding operations.

Every OSI layer has its own unique security challenges and issues, since different layers rely on different protocols, hence exhibiting different security vulnerabilities [68]-[70]. Below we summarize the range of wireless attacks potentially encountered by various protocol layers.

\section{A. Physical-Layer Attacks}

The physical layer is the lowest layer in the OSI protocol architecture, which is used for specifying the physical characteristics of signal transmission. Again, the broadcast nature of wireless communications makes its physical layer extremely vulnerable to eavesdropping and jamming attacks, which are two main types of wireless physical-layer attacks, as depicted in Table 3. More specifically, the eavesdropping attack refers to an unauthorized user attempting to intercept the data transmission between legitimate users [71]. In wireless networks, as long as an eavesdropper lies in the transmit coverage area of the source node, the wireless communications session can be overheard by the eavesdropper. In order to maintain confidential transmission, typically cryptographic techniques relying on secret keys are adopted for preventing eavesdropping attacks from intercepting the data transmission. To be specific, the $\mathrm{SN}$ and the DN

Table 3 Main Types of Wireless Attacks at the PHY Layer

\begin{tabular}{|c|c|}
\hline PHY Attacks & \multicolumn{1}{|c|}{ Characteristics and Features } \\
\hline Eavesdropping & Interception of confidential information [71] \\
\hline Jamming & Interruption of legitimate transmission [72] \\
\hline
\end{tabular}


share a secret key and the so-called plaintext is first encrypted at SN, leading to the cipher text, which is then transmitted to DN. In this case, even if an eavesdropper overhears the cipher text transmission, it remains difficult to extract the plaintext from the cipher text without the secret key.

Moreover, a malicious node in wireless networks can readily generate intentional interference for disrupting the data communications between legitimate users, which is referred to as a jamming attack (also known as DoS attack) [72]. The jammer aims for preventing authorized users from accessing wireless network resources and this impairs the network availability for the legitimate users. To this end, spread spectrum techniques are widely recognized as an effective means of defending against DoS attacks by spreading the transmit signal over a wider spectral bandwidth than its original frequency band. Again, the aforementioned DSSS and FHSS techniques exhibit a high jamming resistance at the physical layer.

\section{B. MAC-Layer Attacks}

The MAC layer enables multiple network nodes to access a shared medium with the aid of intelligent channel access control mechanisms such as CSMA/CA, CDMA, OFDMA, and so on. Typically, each network node is equipped with a NIC and has a unique MAC address, which is used for user authentication. An attacker that attempts to change its assigned MAC address with a malicious intention is termed as MAC spoofing, which is the primary technique of MAC attacks [73]. Although the MAC address is hard-coded into the NIC of a network node, it is still possible for a network node to spoof a MAC address and thus MAC spoofing enables the malicious node to hide its true identity or to impersonate another network node for the sake of carrying out illicit activities. Furthermore, a MAC attacker may overhear the network traffic and steal a legitimate node's MAC address by analyzing the overheard traffic, which is referred to as an identity-theft attack. An attacker attempting identity theft will pretend to be another legitimate network node and gain access to confidential information of the victim node.

In addition to the aforementioned MAC spoofing and identity theft, the class of MAC-layer attacks also includes MITM attacks [74] and network injection [75]. Typically, a MITM attack refers to an attacker that first "sniffs" the network's traffic in order to intercept the MAC addresses of a pair of legitimate communicating nodes, then impersonates the two victims and finally establishes a connection with them. In this way, the MITM attacker acts as a relay between the pair of victims and makes them feel that they are communicating directly with each other over a private connection. In reality, their session was intercepted and controlled by the attacker. By contrast, the network injection attack aims for
Table 4 Main Types of Wireless Attacks at the MAC Layer

\begin{tabular}{|c|c|}
\hline MAC Attacks & Characteristics and Features \\
\hline MAC spoofing & Falsification of MAC address [73] \\
\hline Identity theft & Stealing of a legitimate user's MAC identity \\
\hline MITM attack & Impersonation of a pair of communicating nodes [74] \\
\hline Network injection & Injection of forged network commands and packets [75] \\
\hline
\end{tabular}

preventing the operation of networking devices, such as routers, switches, etc. by injecting forged network reconfiguration commands. In this manner, if an overwhelming number of the forged networking commands are initiated, the entire network may become paralyzed, thus requiring rebooting or even reprogramming of all networking devices. The main types of wireless MAC attacks are summarized in Table 4.

\section{Network-Layer Attacks}

In the network layer, IP was designed as the principal protocol for delivering packets from an $\mathrm{SN}$ to a $\mathrm{DN}$ through intermediate routers based on their IP addresses. The network-layer attacks mainly aim for exploiting IP weaknesses, which include the IP spoofing and hijacking as well as the so-called Smurf attack [76]-[78], as illustrated in Table 5. To be specific, IP spoofing is used for generating a forged IP address with the goal of hiding the true identity of the attacker or impersonating another network node for carrying out illicit activities. The network node that receives these packets associated with a forged source IP address will send its responses back to the forged IP address. This will waste significant network capacity and might even paralyze the network by flooding it with forged IP packets. IP hijacking is another illegitimate activity launched by hijackers for the sake of taking over another legitimate user's IP address. If the attacker succeeds in hijacking the IP address, it will be able to disconnect the legitimate user and create a new connection to the network by impersonating the legitimate user, hence gaining access to confidential information. There are some other forms of IP hijacking

Table 5 Main Types of Wireless Attacks at the Network Layer

\begin{tabular}{|c|c|}
\hline Network Attacks & Characteristics and Features \\
\hline IP spoofing & Falsification of IP address [76] \\
\hline IP hijacking & Impersonation of a legitimate user's IP address [77], [78] \\
\hline Smurf attack & $\begin{array}{r}\text { Paralyzation of a network by launching a huge number } \\
\text { of ICMP requests [79] }\end{array}$ \\
\hline
\end{tabular}


techniques, including prefix hijacking, route hijacking and border gateway protocol hijacking [78].

The Smurf attack is a DoS attack in the network layer, which intends to send a huge number of ICMP packets (with a spoofed source IP address) to a victim node or to a group of victims using an IP broadcast address [79]. Upon receiving the ICMP requests, the victims are required to send back ICMP responses, resulting in a significant amount of traffic in the victim network. When the Smurf attack launches a sufficiently high number of ICMP requests, the victim network will become overwhelmed and paralyzed by these ICMP requests and responses. To defend against Smurf attacks, a possible solution is to configure the individual users and routers by ensuring that they do not to constantly respond to ICMP requests. We may also consider the employment of firewalls, which can reject the malicious packets arriving from the forged source IP addresses.

\section{Transport-Layer Attacks}

This section briefly summarizes the malicious activities in the transport layer, with an emphasis on the TCP and UDP attacks. To be specific, TCP is a connectionoriented transport protocol designed for supporting the reliable transmission of data packets, which is typically used for delivering e-mails and for transferring files from one network node to another. In contrast to TCP, UDP is a connectionless transport protocol associated with a reduced protocol overhead and latency, but as a price, it fails to guarantee reliable data delivery. It is often used by delay-sensitive applications which do not impose strict reliability requirements, such as IP television, voice over IP and online games. Both TCP and UDP suffer from security vulnerabilities including the TCP and UDP flooding as well as the TCP sequence number prediction attacks, as summarized in Table 6.

TCP attacks include TCP flooding attacks and sequence number prediction attacks [80], [81]. The TCP flooding, which is also known as ping flooding, is a DoS attack in the transport layer, where the attacker sends an overwhelming number of ping requests, such as ICMP echo requests to a victim node, which then responds by sending ping replies, such as ICMP echo replies. This will flood both the input and output buffers of the victim node and it might even delay its connection to the target network, when the number of ping requests is sufficiently

Table 6 Main Types of Wireless Attacks at the Transport Layer

\begin{tabular}{|c|c|}
\hline Transport Attacks & Characteristics and Features \\
\hline TCP flooding & Sending a huge number of ping requests [80], [81] \\
\hline UDP flooding & Launching an overwhelming number of UDP packets [82] \\
\hline $\begin{array}{c}\text { TCP sequence } \\
\text { prediction attack }\end{array}$ & $\begin{array}{r}\text { Fabrication of a legitimate user's data packets using the } \\
\text { predicted TCP sequence index }\end{array}$ \\
\hline
\end{tabular}

high. The TCP sequence prediction technique is another TCP attack that attempts to predict the sequence index of TCP packets of a transmitting node and then fabricates the TCP packets of the node. To be specific, the TCP sequence prediction attacker first guesses the TCP sequence index of a victim transmitter, then fabricates packets using the predicted TCP index, and finally sends its fabricated packets to a victim receiver. Naturally, the TCP sequence prediction attack will inflict damage upon the data integrity owing to the aforementioned packet fabrication and injection.

The UDP is also prone to flooding attacks, which are imposed by sending an overwhelming number of UDP packets, instead of ping requests used in the TCP flood attack. Specifically, a UDP flood attacker transmits a large number of UDP packets to a victim node, which will be forced to send numerous reply packets [82]. In this way, the victim node will be overwhelmed by the malicious UDP packets and becomes unreachable by other legitimate nodes. Moreover, the UDP flooding attacker is capable of hiding itself from the legitimate nodes by using a spoofed IP address for generating malicious UDP packets. The negative impact of such UDP flooding attacks is mitigated by limiting the response rate of UDP packets. Furthermore, firewalls can be employed for defending against the UDP flooding attacks for filtering out malicious UDP packets.

\section{E. Application-Layer Attacks}

As mentioned above, the application layer supports HTTP [62] for web services, FTP [83] for file transfer and SMTP [84] for e-mail transmission. Each of these protocols is prone to security attacks. Logically, the application-layer attacks may hence be classified as HTTP attacks, FTP attacks, and SMTP attacks. More specifically, HTTP is the application protocol designed for exchanging hypertext across the World Wide Web, which is subject to numerous security threats. The main HTTP attacks include the malware attack (e.g., Trojan horse, viruses, worms, backdoors, keyloggers, etc.), structured query language (SQL) injection attack, and cross-site scripting attack [85]. The terminology "malware" refers to malicious software which is in the form of code, scripts, and active content programmed by attackers attempting to disrupt legitimate transmissions or to intercept confidential information. The SQL injection is usually exploited to attack data-driven applications by inserting certain rogue SQL statements with an attempt to gain unauthorized access to legitimate websites. The last type of HTTP attacks to be mentioned is referred to as cross-site scripting attacks that typically occur in web applications and aim for bypassing some of the access control measures (e.g., the same origin policy) by injecting client-side scripts into web pages [85].

The FTP is used for large-file transfer from one network node to another, which also exhibits certain 
Table 7 Main Types of Wireless Attacks at the Application Layer

\begin{tabular}{|c|l|}
\hline Application Attacks & \multicolumn{1}{|c|}{ Characteristics and Features } \\
\hline Malware attack & $\begin{array}{l}\text { Malicious software in the form of code, scripts and } \\
\text { active content programmed by attackers [85] }\end{array}$ \\
\hline SQL injection & $\begin{array}{l}\text { Inserting rogue SQL statements attempting to gain } \\
\text { unauthorized access to legitimate websites }\end{array}$ \\
\hline Cross-site scripting & $\begin{array}{l}\text { Injecting client-side scripts into web pages for by- } \\
\text { passing some of the access control measures }\end{array}$ \\
\hline FTP bounce & $\begin{array}{l}\text { Impersonating a legtimate user to gain unauthorized } \\
\text { access [83] }\end{array}$ \\
\hline SMTP attack & $\begin{array}{l}\text { Malicious attacks in e-mail transfering between the } \\
\text { SMTP servers and clients }\end{array}$ \\
\hline
\end{tabular}

security vulnerabilities. The FTP bounce attacks and directory traversal attacks often occur in FTP applications [83]. The FTP bounce attack exploits the PORT command in order to request access to ports through another victim node, acting as a middle man. We note, however, that most modern FTP servers are configured by default to refuse PORT commands in order to prevent FTP bounce attacks. The directory traversal attack attempts to gain unauthorized access to legitimate file systems by exploiting any potential security vulnerability during the validation of user-supplied input file names. In contrast to FTP, the SMTP is an application-layer protocol designed for transferring e-mails across the Internet, which, however, does not encrypt private information, such as the login username, the password, and the messages themselves transmitted between the SMTP servers and clients, hence raising a serious privacy concern. Moreover, e-mails are frequent carriers of viruses and worms. Thus, the SMTP attacks include the password "sniffing," SMTP viruses, and worms as well as e-mail spoofing [84]. Typically, antivirus software or firewalls (or both) are adopted for identifying and guarding against the aforementioned application-layer attacks. Table 7 summarizes the aforementioned main attacks at the application layer.

Finally, we summarize the similarities and differences between the wireless and wired networks in terms of their security attacks at the different OSI layers. As shown in Fig. 4, the application-, transport-, and network-layer attacks of wireless networks are the same as those of wired networks, since the wireless and wired networks share common protocols at the application, transport and network layers. By contrast, wireless networks are different from wired networks in terms of the PHY and MAC attacks. In general, only the PHY and MAC layers are specified in wireless networking standards (e.g., Wi-Fi, Bluetooth, LTE, etc.). In wireless networks, conventional security protocols are defined at the MAC layer (sometimes at the logical-link-control layer) for establishing a trusted and confidential link, which will be summarized for different commercial

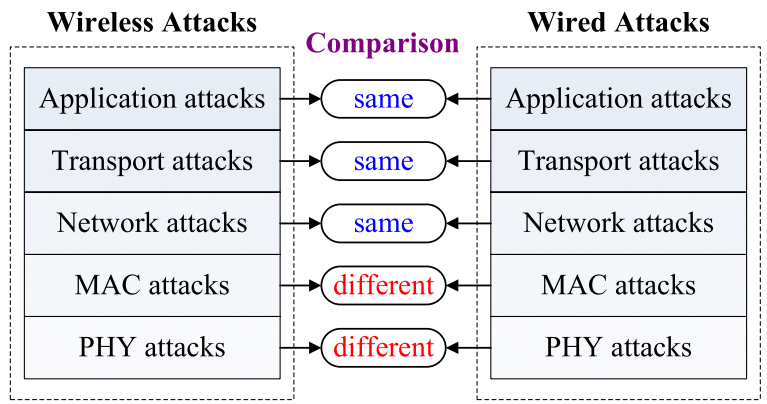

Fig. 4. Comparison between the wireless and wired networks in terms of security attacks at different osı layers.

wireless networks in Section IV. Additionally, the wireless PHY layer is completely different from its wirelinebased counterpart. Due to the broadcast nature of radio propagation, the wireless PHY layer is extremely vulnerable to both the eavesdropping and jamming attacks. To this end, physical-layer security is emerging as an effective means of securing wireless communications against eavesdropping, as will be discussed in Section V. Next, Section VI will present various wireless jamming attacks and their countermeasures.

\section{SECURITY DEFENSE PROTOCOLS AND PARADIGMS FOR WIRELESS NETWORKS}

This section is focused on the family of security protocols and paradigms that are used for improving the security of wireless networks. As compared to wired networks, the wireless networks have the advantage of avoiding the deployment of a costly cable-based infrastructure. The stylized illustration of operational wireless networks is shown in Fig. 5, where the family of WPANs, WLANs, and WMANs are illustrated, which complement each other with the goal of providing users with ubiquitous broadband wireless services [86]. The objective of Fig. 5 is to provide a comparison among the WPAN, WLAN, and WMAN techniques from different perspectives in terms of their industrial standards, coverage area and peak data rates. More specifically, a WPAN is typically used for interconnecting with personal devices (e.g., a keyboard, audio headset, printer, etc.) at a relatively low data rate and within a small coverage area. For example, Bluetooth is a common WPAN standard using shortrange radio coverage in the industrial, scientific, and medical band spanning the band 2400-2480 $\mathrm{MHz}$, which can provide a peak data rate of $2 \mathrm{Mb} / \mathrm{s}$ and a range of up to $100 \mathrm{~m}$ [87]. Fig. 5 also shows that a WLAN generally has a higher data rate and a wider coverage area than the WPAN, which is used for connecting wireless devices through an AP within a local coverage area. As an example, IEEE 802.11 (also known as Wi-Fi) 


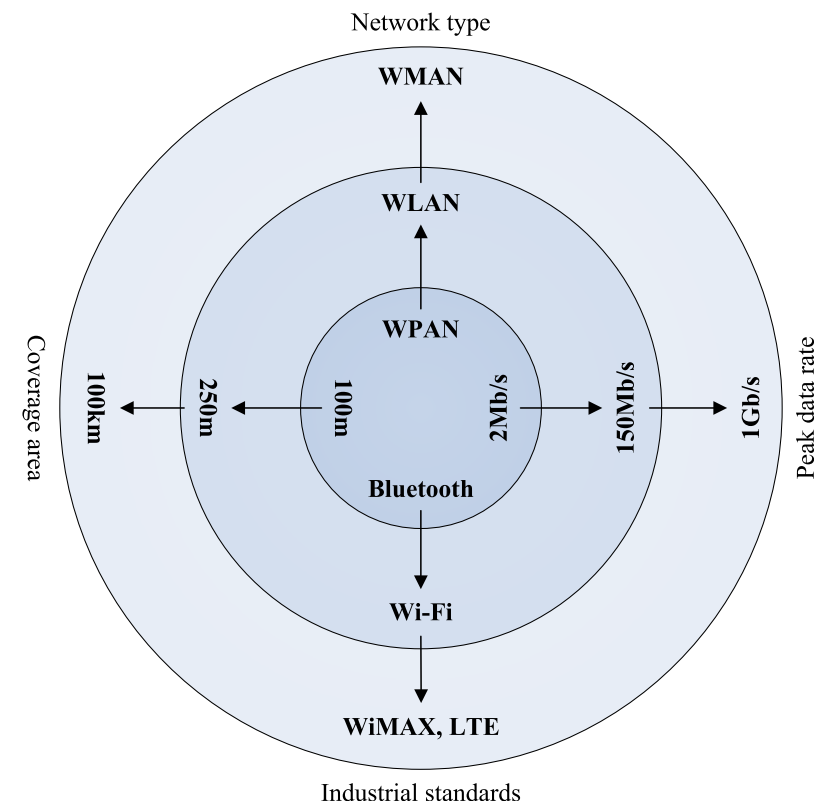

Fig. 5. Family of wireless networks consisting of WPAN, WLAN, and WMAN.

consists of a series of industrial WLAN standards. Modern Wi-Fi standards are capable of supporting a peak data rate of $150 \mathrm{Mb} / \mathrm{s}$ and a maximum range of $250 \mathrm{~m}$ [88]. Finally, a MAN is typically used for connecting a metropolitan city at a higher rate and over a lager coverage area than the WPAN and WLAN. For instance, in Fig. 5, we feature two types of industrial standards for WMAN, namely WiMAX and LTE [89], [90].

In the following, we will present an overview of the security protocols used in the aforementioned wireless standards (i.e., the Bluetooth, Wi-Fi, WiMAX, and LTE) for protecting the authenticity, confidentiality, integrity, and availability of legitimate transmissions through the wireless propagation medium.

\section{A. Bluetooth}

Bluetooth is a short-range and low-power wireless networking standard, which has been widely implemented in computing and communications devices as well as in peripherals, such as cell phones, keyboards, audio headsets, etc. However, Bluetooth devices are subject to a large number of wireless security threats and may easily become compromised. As a protection, Bluetooth introduces diverse security features and protocols for guaranteeing its transmissions against potentially serious attacks [91]. For security reasons, each Bluetooth device has four entities [92], including the Bluetooth device address (BD_ADDR), private authentication key, private encryption key and a random number (RAND), which are used for authentication, authorization and encryption, respectively. More specifically, the BD_ADDR

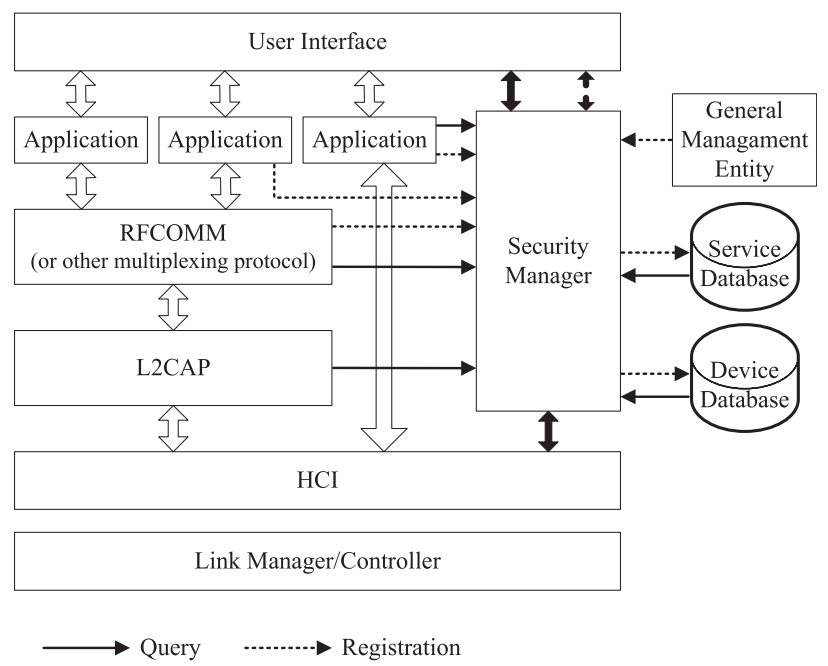

RFCOMM: Radio Frequency Communication

L2CAP: Logical Link Control and Adaptation Protocol

HCI: Host Controller Interface

Fig. 6. Bluetooth security architecture.

contains $48 \mathrm{~b}$, which is unique for each Bluetooth device. The 128-b private authentication key is used for authentication and the private encryption key that varies from 8 to $128 \mathrm{~b}$ in length is used for encryption. In addition, RAND is a frequently changing 128-b pseudorandom number generated by the Bluetooth device itself.

Fig. 6 illustrates the Bluetooth security architecture, where the key component is the security manager responsible for authentication, authorization, and encryption [91]. As shown in Fig. 6, the service database and the device database are mainly used for storing the security-related information on services and devices, respectively, which can be adjusted through the user interface. These databases can also be administrated by the general management entity. When a Bluetooth device receives an access request from another device, it will first query its security manager with the aid of its RFCOMM or other multiplexing protocols. Then, the security manager has to respond to the query as to whether to allow the access by checking both the service database and device database. The generic access profile of Bluetooth defines three security modes:

1) security mode 1 (nonsecure), where no security procedure is initiated;

2) security mode 2 (service-level enforced security), where the security procedure is initiated after establishing a link between the Bluetooth transmitter and receiver;

3) security mode 3 (link level enforced security), where the security procedure is initiated before the link's establishment [91]. 


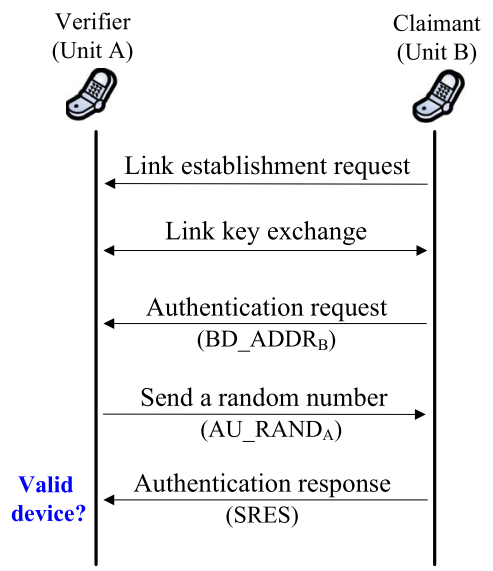

Fig. 7. Bluetooth authentication process.

In Bluetooth systems, a device is classified into one of three categories: trusted/untrusted device, authenticated/ unauthenticated device, and unknown device. The trusted device category implies that the device has been authenticated and authorized as a trusted and fixed relationship, hence has unrestricted access to all services. By contrast, the untrusted device category refers to the fact that the device has indeed been authenticated successfully, but has no permanent fixed relationship, hence it is restricted to specific services. If a Bluetooth device is successfully authenticated, but has not completed any authorization process, it will be considered as an authenticated device. By definition, an unauthenticated device failed to authenticate and has a limited access to services. If a device has not passed any authentication and authorization process, it is classified as an unknown device and hence it is restricted to access services requiring the lowest privilege. Additionally, the Bluetooth services are also divided into the following three security levels: 1) authorization-level services, which can be accessed by trusted devices only; 2) authentication-level services, which require authentication, but no authorization, hence, they remain inaccessible to the unauthenticated devices and unknown devices; and 3) open services, which are open to access by all devices. Below, we would like to discuss the detailed procedures of authentication, authorization, and encryption in Bluetooth.

The authentication represents the process of verifying the identity of Bluetooth devices based on the BD_ADDR and link key. As shown in Fig. 7, the Bluetooth authentication adopts a "challenge-response scheme" [93], where the verifier (Unit A) challenges the claimant (Unit B) which then responds for the sake of authentication. To be specific, the claimant first requests the verifier to establish a link and then exchanges a link key that is a 128-b random number. Next, an authentication request with the claimant's address $\mathrm{BD} \_\mathrm{ADDR} \mathrm{B}_{\mathrm{B}}$ is sent to the

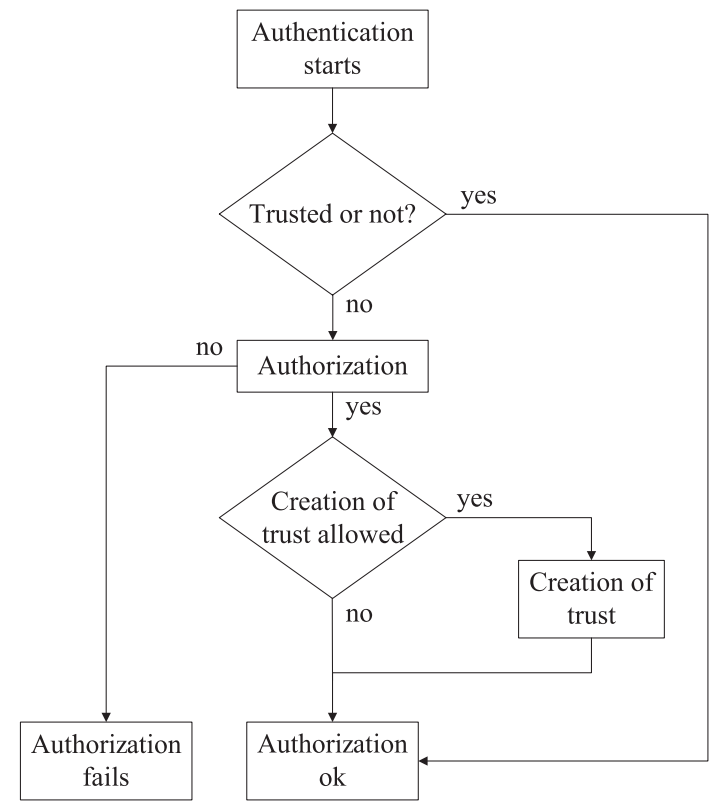

Fig. 8. Flow chart of Bluetooth authorization.

verifier, which returns a random number denoted by AU_RAND ${ }_{A}$. Then, both the verifier and claimant perform the same authentication function using the random number AU_RAND ${ }_{A}$, the claimant's address $B D \_A D D R_{B}$, and the link key to obtain their responses denoted by SRES' and SRES, respectively. Finally, the claimant sends its response SRES to the verifier, which will compare SRES with its own response SRES'. If SRES is identical to SRES', the authentication is confirmed. By contrast, a mismatch between SRES' and SRES represents an authentication failure.

The authorization process is used for deciding whether a Bluetooth device has the right to access a certain service. Typically, trusted devices are allowed to access all services, however untrusted or unknown devices require authorization, before their access to services is granted. Fig. 8 shows a flow chart of the Bluetooth authorization process. Observe from Fig. 8 that the authorization process commences with checking the device database for deciding whether the Bluetooth device was authorized previously and considered trusted. If the Bluetooth device is trusted, the authorization is concluded. Otherwise, the authorization and the trust-creation will be performed sequentially. If the authorization fails, the access to certain services will be denied. Meanwhile, a successful authorization makes the corresponding Bluetooth devices trustworthy for accessing all services.

Additionally, encryption is employed in Bluetooth to protect the confidentiality of transmissions. The payload of a Bluetooth data packet is encrypted by using a stream cipher, which consists of the payload key generator and 
key stream generator [93]. To be specific, first a payload key is generated with the aid of the link key and Bluetooth device address, which is then used for generating the key stream. Finally, the key stream and plaintext are added in modulo- 2 in order to obtain the cipher text. It is pointed out that the payload key generator simply combines the input bits in an appropriate order and shifts them to four linear feedback shift registers to obtain the payload key. Moreover, the key stream bits are generated by using a method derived from the summation stream cipher generator by Toengel [93].

\section{B. Wi-Fi}

The family of Wi-Fi networks mainly based on the IEEE $802.11 \mathrm{~b} / \mathrm{g}$ standards has been explosively expanding. The most common security protocols in Wi-Fi are referred to as WEP and WPA [94]. WEP was proposed in 1999 as a security measure for Wi-Fi networks to make wireless data transmissions as secure as in traditional wired networks. However, WEP has been shown to be a relatively weak security protocol, having numerous flaws. Hence, it can be "cracked" in a few minutes using a basic laptop computer. As an alternative, WPA was put forward in 2003 for replacing WEP, while the improved WPA2 constitutes an upgraded version of the WPA standard. Typically, WPA and WPA2 are more secure than WEP and thus they are widely used in modern Wi-Fi networks. Below, we detail the authentication and encryption processes of the WEP, WPA and WPA2 protocols.

The WEP protocol consists of two main parts, namely the authentication part and encryption part, aiming for establishing access control by preventing unauthorized access without an appropriate WEP key and hence they achieve data privacy by encrypting the data streams with the aid of the WEP key. As shown in Fig. 9, the WEP authentication uses a four-step "challenge-response" handshake between a Wi-Fi client and an access point operating with the aid of a shared WEP key. To be specific, the client first sends an authentication request to the access point, which then replies with a plaintext

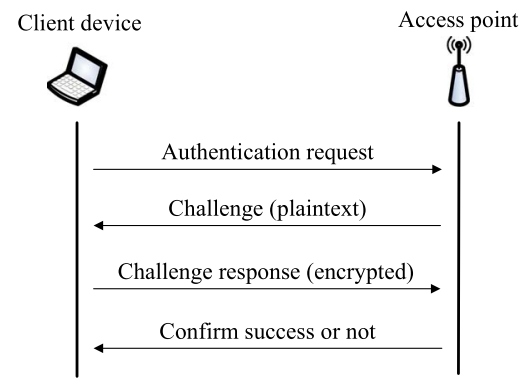

Fig. 9. WEP authentication process.

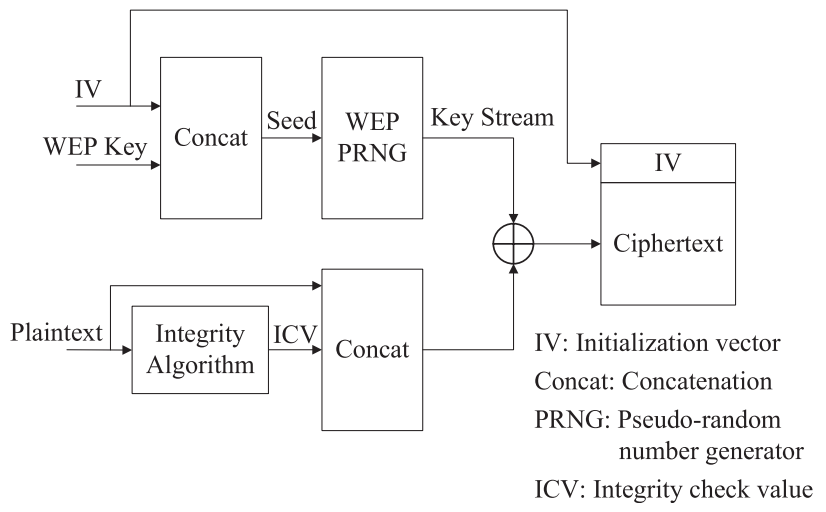

Fig. 10. Block diagram of WEP encryption.

challenge. After that, the client encrypts its received "challenge text" using a preshared WEP key and sends the encrypted text to the access point. It then decrypts the received encrypted text with the aid of the preshared WEP key and attempts to compare the decrypted text to the original plaintext. If a match is found, the access point sends a successful authentication indicator to the client. Otherwise, the authentication is considered as failed.

Following the authentication, WEP activates the process of encrypting data streams using the simple Rivest Cipher 4 Algorithm operating with the aid of the preshared WEP key [96]. Fig. 10 shows a block diagram of the WEP encryption, where first an initialization vector (IV) of $24 \mathrm{~b}$ is concatenated to a 40-b WEP key. This leads to a 64-b seed for a PRNG, which is then used for generating the key stream. Additionally, an integrity check algorithm is performed such as a cyclic redundancy check on the plaintext in order to obtain an ICV, which can then be used for protecting the data transmission from malicious tampering. Then, the ICV is concatenated with the plaintext, which will be further combined with the aforementioned key stream in modulo-2 for generating the cipher text. Although WEP carries out both the authentication and encryption functions, it still remains prone to security threats. For example, WEP fails to protect the information against forgery and replay attacks, hence an attacker may be capable of intentionally either modifying or replaying the data packets without the legitimate users becoming aware that data falsification and/or replay has taken place. Furthermore, the secret keys used in WEP may be "cracked" in a few minutes using a basic laptop computer [97]. Additionally, it is easy for an attacker to forge an authentication message in WEP, which makes it straightforward for unauthorized users to pretend to be legitimate users and hence to steal confidential information [98].

As a remedy, WPA was proposed for addressing the aforementioned WEP security problems, which was 


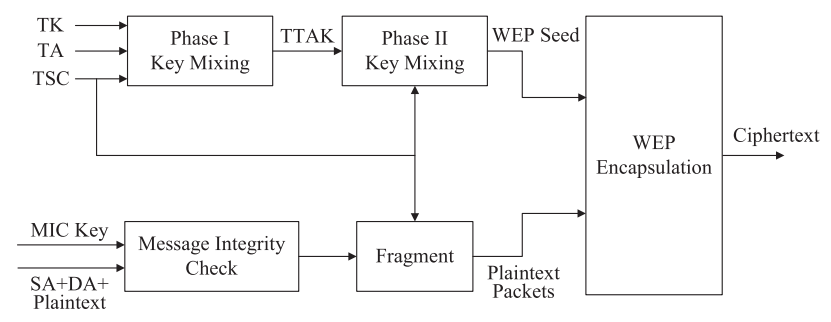

Fig. 11. Illustration of TKIP encryption process.

achieved by Wi-Fi users without the need of changing their hardware. The WPA standard has two main types:

1) personal WPA is mainly used in home without the employment of an authentication server, where a secret key is preshared between the client and access point, which is termed as WPA-PSK (preshared key);

2) enterprise WPA used for enterprise networks, which requires an authentication server $802.1 \mathrm{x}$ for carrying out the security control in order to effectively guard against malicious attacks.

The main advantage of WPA over WEP is that WPA employs more powerful data encryption referred to as the TKIP, which is assisted by a MIC invoked for the sake of protecting the data integrity and confidentiality of Wi-Fi networks [99], [100]. Fig. 11 shows the TKIP encryption process, in which the TA, the TK, and the TSC constitute the inputs of the phase I key mixing process, invoked in order to obtain a so-called TTAK, which is then further processed along with the TSC in the phase II key mixing stage for deriving the WEP seed, including a WEP IV and a base key. Furthermore, observe in Fig. 11 that the MIC is performed both on the SA, as well as on the DA and the plaintext. The resultant MIC will then be appended to the plaintext, which is further fragmented into multiple packets, each assigned with a unique TSC. Finally, the WEP seed and plaintext packets are used for deriving the cipher text by invoking the WEP encryption, as discussed in Fig. 10, which is often implemented in the hardware of Wi-Fi devices. We note that even the WPA relying on the TKIP remains vulnerable to diverse practical attacks [101].

WiMAX (also known as IEEE 802.16) is a standard developed for WMAN and the initial WiMAX system was designed for providing a peak data rate of $40 \mathrm{Mb} / \mathrm{s}$. In order to meet the requirements of the International Mobile Telecommunications-Advanced initiative, IEEE $802.16 \mathrm{~m}$ was proposed as an updated version of the original WiMAX, which is capable of supporting a peak data rate of $1 \mathrm{~Gb} / \mathrm{s}$ for stationary reception and $100 \mathrm{Mb} / \mathrm{s}$ for mobile reception [102]. As all other wireless systems, WiMAX also faces various wireless attacks and provides

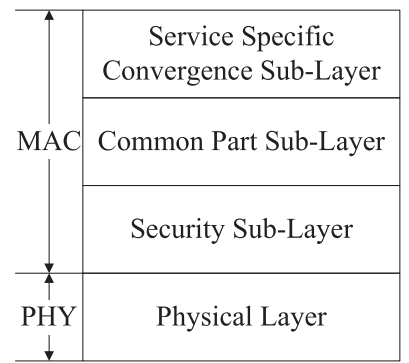

(a)

\begin{tabular}{|c|c|c|}
\hline $\begin{array}{c}\text { RSA-based } \\
\text { Authentication }\end{array}$ & $\begin{array}{c}\text { Authenticatio/SA } \\
\text { Control }\end{array}$ & $\begin{array}{c}\text { EAP Encapsulation/ } \\
\text { Decapsulation }\end{array}$ \\
\hline \multicolumn{2}{|c|}{ PKM Control Management } \\
\hline $\begin{array}{c}\text { Traffic Data Encryption/ } \\
\text { Authentication Processing }\end{array}$ & Control Message Processing \\
\cline { 2 - 2 } & $\begin{array}{c}\text { Message Authentication } \\
\text { Processing }\end{array}$ \\
\hline
\end{tabular}

(b)

Fig. 12. WiMAX protocol stack: (a) PHY-MAC illustration; and (b) security sublayer specification.

advanced features for enhancing the attainable transmission security. To be specific, a security sub-layer is introduced in the protocol stack of the WiMAX standard, as shown in Fig. 12 [103].

\section{WiMAX}

It is observed from Fig. 12(a) that the protocol stack of a WiMAX system defines two main layers, namely the physical layer and the MAC layer. Moreover, the MAC layer consists of three sublayers, namely the servicespecific convergence sublayer, the common part sublayer, and the security sublayer. All the security issues and risks are considered and addressed in the security sublayer. Fig. 12(b) shows the WiMAX security sublayer, which will be responsible for authentication, authorization, and encryption in WiMAX networks. The security sublayer defines a so-called PKM protocol, which considers the employment of the X.509 digital certificate along with the RSA public-key algorithm and the AES algorithm for both user authentication as well as for key management and secure transmissions. The initial PKM version (PKMv1) as specified in early WiMAX standards (e.g., IEEE 802.16a/c) employs an unsophisticated oneway authentication mechanism and hence it is vulnerable to MITM attacks. To address this issue, an updated PKM version (PKMv2) was proposed in the more sophisticated WiMAX standard releases (e.g., IEEE 802.16e/m) [104], which relies on two-way authentication. The following discussions detail both the WiMAX authentication as well as the authorization and encryption processes. 


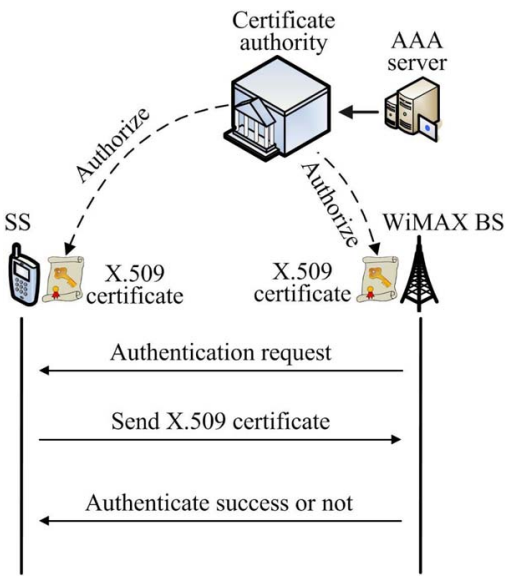

Fig. 13. RSA-based authentication process.

Authentication in WiMAX is achieved by the PKM protocol, which supports two basic authentication approaches, namely the RSA-based authentication and the EAP-based authentication [105]. Fig. 13 shows the RSAbased authentication process, where a trusted certificate authority is responsible for issuing an X.509 digital certificate to each of the network nodes, including the SS and the WiMAX BS. An X.509 certificate contains both the public key and the MAC address of its associated network node. During the RSA-based authentication process shown in Fig. 13, when an SS receives an authentication request from a WiMAX BS, it sends its X.509 digital certificate to the $\mathrm{BS}$, which then verifies whether the certificate is valid. If the certificate is valid, the SS is considered authenticated. By contrast, an invalid certificate implies that the SS fails to authenticate.

The EAP-based authentication process is illustrated in Fig. 14, where a WiMAX BS first sends an identity request to an SS who responds with its identity information. The WiMAX BS then forwards the SS's identity to an AAA server over a secure networking protocol referred to as RADIUS. After that, the SS and the AAA server start the authentication process, where three different EAP options are available depending on the SS and AAA server's capability, including the EAP-AKA, EAP-TLS, and EAP-TTLS. Finally, the AAA server will indicate the success (or failure) of the authentication and notify the SS.

Additionally, the authorization process is necessary for deciding whether an authenticated SS has the right to access certain WiMAX services [106]. In the WiMAX authorization process, an SS first sends an authorization request message to the BS that contains both the SS' X. 509 digital certificate, as well as the encryption algorithm and the cryptographic identity (ID). After receiving the authorization request, the BS validates the SS' request by interacting with an AAA server and then

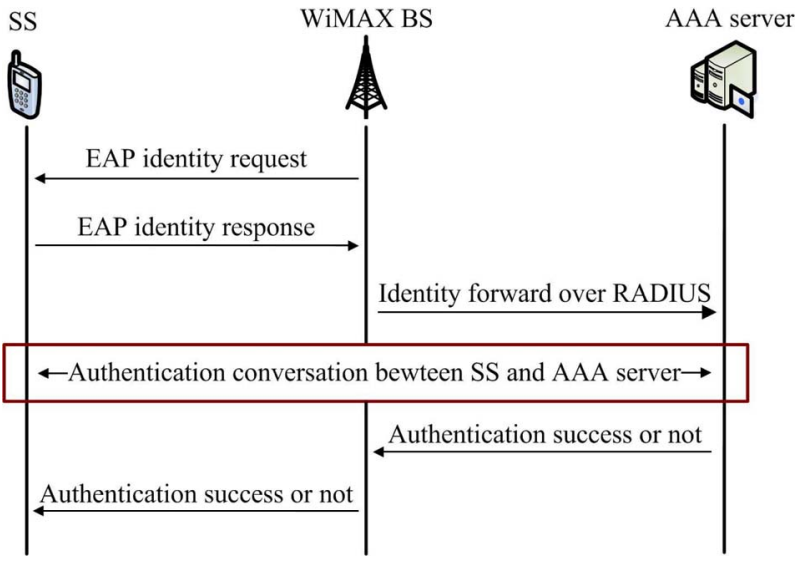

Fig. 14. EAP-based authentication process.

sends back an authorization reply to the SS. Once the positive authorization is confirmed, the SS will be allowed to access its intended services. Following user authentication and authorization, the SS is free to exchange data packets with the BS. In order to guarantee transmission confidentiality, WiMAX considers the employment of the AES algorithm for data encryption, which is much more secure than the DES algorithm [107]. Unlike the DES that uses the Feistel cipher design principles of [107], the AES cipher is based on a socalled substitution-permutation network and has a variable block size of 128,192 , or 256 b [108]. This key length specifies the number of transformation stages used for converting the plaintext into cipher text. In WiMAX, the AES algorithm supports several different modes, including the cipher-block chaining mode, counter mode, and electronic codebook mode.

\section{Long-Term Evolution}

LTE is the most recent standard developed by the $3 \mathrm{G}$ partnership project for next-generation mobile networks designed for providing seamless coverage, high data rate, and low latency [109]. It supports packet switching for seamless interworking with other wireless networks and also introduces many new elements, such as relay stations, home eNodeB (HeNB) concept, etc. An LTE network typically consists of an EPC and an E-UTRAN, as shown in Fig. 15 [90], [110]. The EPC comprises an MME, a serving gateway, a packet data network gateway (PDN GW), and an HSS. Moreover, the E-UTRAN includes a base station (also termed as eNodeB in LTE) and several UE. If channel conditions between the UE and eNodeB are poor, a relay station may be activated for assisting their data communications. Furthermore, both in small offices and in residential environments, a HeNB may be installed for improving the indoor coverage by increasing both the capacity and reliability of the E-UTRAN. Although introducing these elements into 


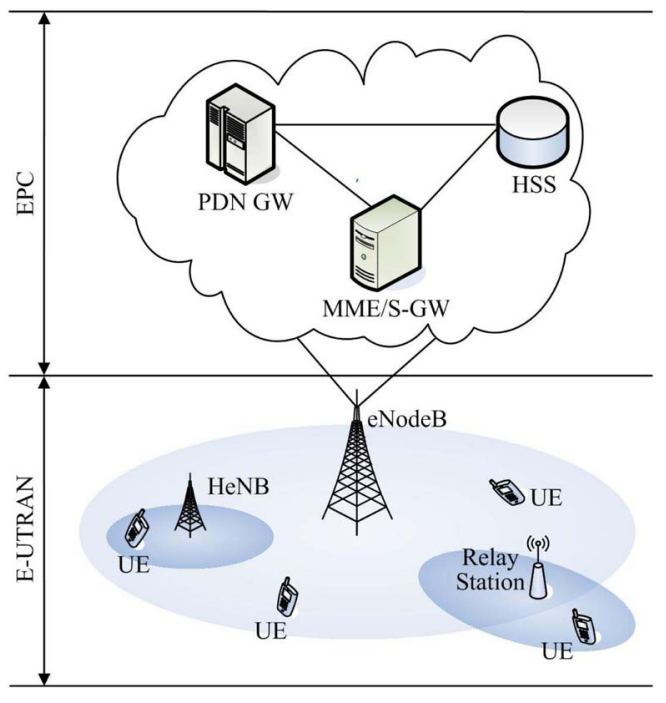

Fig. 15. LTE network architecture.

LTE is capable of improving the network coverage and quality, it has its own new security vulnerabilities and threats.

In order to facilitate secure packet exchange between the UE and EPC, a so-called EPS-AKA protocol was proposed for defending LTE networks against various attacks, including redirection attacks, rogue base station attacks [111], and MITM attacks. A two-way authentication process was invoked between the UE and EPC, which is adopted in the EPS-AKA protocol responsible for generating both the CKs and the IKs [112]. Both the CKs and the IKs are used for data encryption and integrity check for enhancing the confidentiality and integrity of LTE transmissions. Fig. 16 shows this two-way authentication process of the LTE system using the EPS-AKA protocol, where an UE and an LTE network should validate each other's identity.

To be specific, the MME first sends a user identity verification request to the UE that then replies back with its unique IMSI. Next, the MME sends an authentication data request to HSS, which consists of the UE's IMSI and the serving network's identity. Upon receiving the request, the HSS responds to the MME by sending back an EPS authentication vector containing the quantities (RAND, XRES, AUTN, KSI ASME), where RAND is an input parameter, while XRES is an output of the authentication algorithm at the LTE network side. Furthermore, AUTN indicates the identifier of the network authority, while KSI ASME is the key set identity of the access security management entity. Then, the MME sends an authentication request to the UE containing the RAND, AUTN, and KSI ASME quantities. As a result, the UE checks its received parameter AUTN for authenticating the LTE network. If the network authentication is successful, the UE generates the response RES and sends it

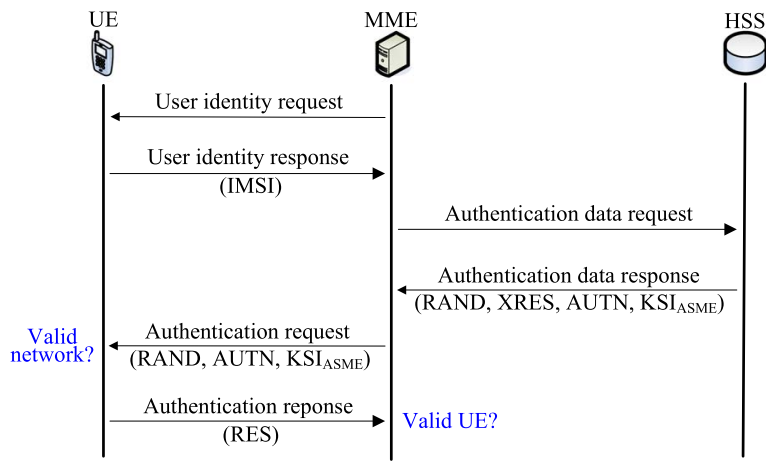

Fig. 16. Two-way authentication in LTE by using the EPS-AKA protocol.

to the MME, which compares XRES with RES. If XRES is the same as RES, this implies that the UE also passes the authentication.

In the UMTS, also known as the 3G mobile cellular system, KASUMI [113] is used as the ciphering algorithm for protecting the data confidentiality and integrity, which, however, has several security weaknesses and hence it is vulnerable to certain attacks, such as the related-key attack [113]. To this end, the LTE system adopts a more secure ciphering technique referred to as SNOW 3G [114] that is a block-based ciphering solution used as the heart of LTE confidentiality and integrity algorithms, which are referred to as the UEA2 and UIA2, respectively [114]. The SNOW 3G technique is referred to as a stream cipher having two main components, namely an internal state of $608 \mathrm{~b}$ controlled by a 128-b key and a 128-b IV, which are utilized for generating the cipher text by masking the plaintext. During the SNOW 3G operation process, we first perform key initialization to make the cipher synchronized to a clock signal and a 32-b key stream word is produced in conjunction with every clock.

In summary, in Sections IV-A-IV-D, we have discussed the security protocols of Bluetooth, Wi-Fi, WiMAX as well as of LTE and observed that the existing wireless networks tend to rely on security mechanisms deployed at the upper OSI layers of Fig. 2 (e.g., MAC layer, network layer, transport layer, etc.) for both user authentication and data encryption. For example, the WEP and WPA constitute a pair of security protocols commonly used in Wi-Fi networks for guaranteeing the data confidentiality and integrity requirements, whereas WiMAX networks adopt the PKM protocol for achieving secure transmissions in the face of malicious attacks. By contrast, communication security at physical layer has been largely ignored in existing wireless security protocols. However, due to the broadcast nature of radio propagation, the physical layer of wireless transmission is extremely vulnerable to both eavesdropping and jamming attacks. This 


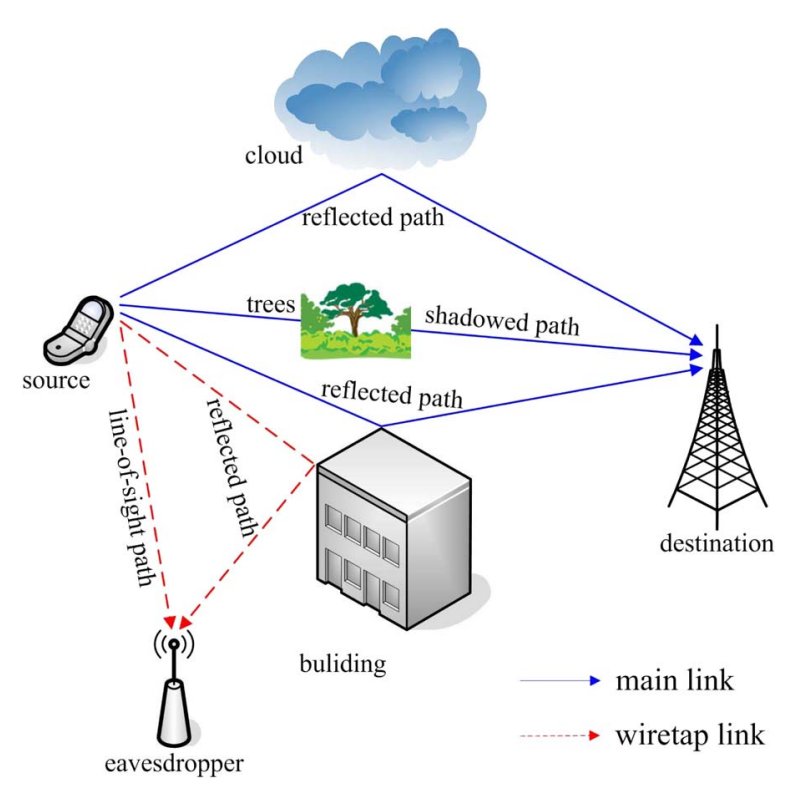

Fig. 17. Wireless scenario transmitting from source to destination in multipath fading environments in the presence of an eavesdropper.

necessitates the development of physical-layer security as a complement to conventional upper-layer security protocols. The following section will introduce the physicallayer security paradigm conceived for facilitating secure wireless communications.

\section{WIRELESS PHYSICAL-LAYER SECURITY AGAINST EAVESDROPPING}

In this section, we portray the field of wireless physicallayer security, which has been explored for the sake of enhancing the protection of wireless communications against eavesdropping attacks. Fig. 17 shows a wireless scenario transmitting from a source to a destination in the presence of an eavesdropper, where the main and wiretap links refer to the channels spanning from the source to the destination and to the eavesdropper, respectively. As shown in Fig. 17, when a radio signal is transmitted from the source, multiple differently delayed signals will be received at the destination via different propagation paths due to the signal reflection, diffraction and scattering experienced. Owing to the multipath effects, the differently delayed signal components sometimes add constructively, sometimes destructively. Hence, the attenuation of the signal that propagated through the space fluctuates in time, which is referred to as fading and it is usually modeled as a random process. The signal received at the destination may be attenuated significantly, especially when a deep fade is encountered, due to the shadowing in the presence of obstacles (e.g., trees) between the source and destination. Moreover, due to the broadcast nature of radio propagation, the source signal may be overheard by the eavesdropper, which also experiences a multipath fading process. There are three typical probability distribution models routinely used for characterizing the random wireless fading, including the Rayleigh fading [115], Rice fading [116], and Nakagami fading [117].

Recently, physical-layer security has been emerging as a promising paradigm designed for improving the security of wireless transmissions by exploiting the physical characteristics of wireless channels [33], [34], [118]. More specifically, it was shown in [33] that reliable information-theoretic security can be achieved, when the wiretap channel spanning from the source to the eavesdropper is a degraded version of the main channel between the source and the destination. In [34], a so-called secrecy capacity was developed and shown as the difference between the capacity of the main channel and that of the wiretap channel, where a positive secrecy capacity means that reliable information-theoretic security is possible and vice versa. However, in contrast to wired channels that are typically time invariant, wireless channels suffer from time-varying random fading, which results in a significant degradation of the wireless secrecy capacity [119], especially when a deep fade is encountered in the main channel due to shadowing by obstacles (e.g., buildings, trees, etc.) appearing between the source and the destination. Hence considerable research efforts have been devoted to the development of various physicallayer security techniques, which can be classified into the following main research categories:

1) information-theoretic security [119]-[125];

2) artificial-noise-aided security [126]-[130];

3) security-oriented beamforming techniques [131]-[136];

4) diversity-assisted security approaches [42], [137];

5) physical-layer secret key generation [147]-[161].

The aforementioned physical-layer security techniques are summarized in Fig. 18. In the following, we will detail these physical-layer security topics.

\section{A. Information-Theoretic Security}

Information-theoretic security examines fundamental limits of physical-layer security measures from an information-theoretic perspective. The concept of information-theoretic security was pioneered by Shannon in [119], where the basic theory of secrecy systems was developed with an emphasis on the mathematical structure and properties. To be specific, Shannon defined a secrecy system as a set of mathematical transformations of one space (the set of legitimate plaintext messages) into another space (the set of possible cryptograms), where each transformation corresponds to enciphering the information with the aid of a secret key. Moreover, 


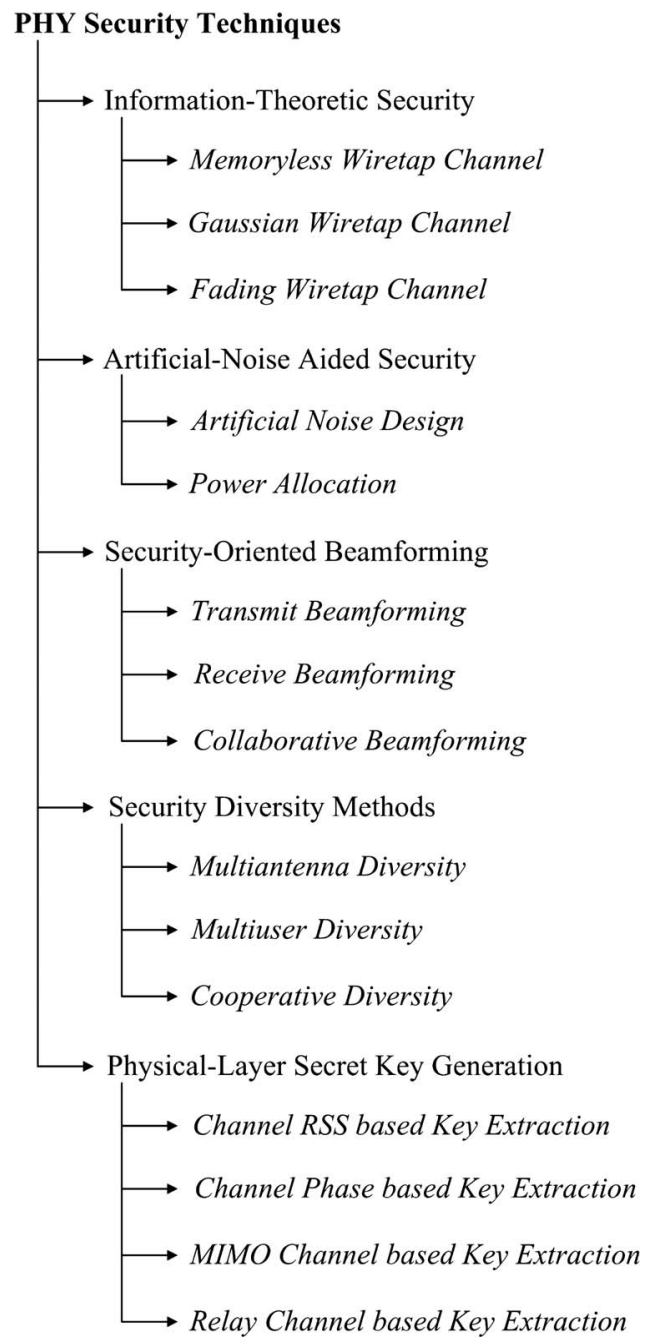

Fig. 18. Classification of physical-layer security techniques.

the transformation is nonsingular so that unique deciphering becomes possible, provided that the secret key is known. In [119], the notions of theoretical secrecy and practical secrecy were introduced, which was developed for the ease of guarding against eavesdropping attacks, when an adversary is assumed to have either infinite or more practically finite computing power. It was shown in [119] that a perfect secrecy system may be created, despite using a finite-length secret key, where the equivocation at the adversary does not approach zero, i.e., when the adversary is unable to obtain a unique solution to the cipher text. To elaborate a little further, the equivocation is defined as a metric of quantifying how uncertain the adversary is of the original cipher text after the act of message interception [119].

The secrecy system developed by Shannon in [119] is based on the employment of secret keys. However, the key management is challenging in certain wireless networks operating without a fixed infrastructure (e.g., wireless ad hoc networks) [32]. To this end, in [33], Wyner investigated the information-theoretic security without using secret keys and examined its performance limits for a discrete memoryless wiretap channel consisting of a source, a destination and an eavesdropper. It was shown in [33] that perfectly secure transmission can be achieved, provided that the channel capacity of the main link spanning from the SN to its DN is higher than that of the wiretap link between the SN and eavesdropper. In other words, when the main channel conditions are better than the wiretap channel conditions, there exists a positive rate at which the $\mathrm{SN}$ and DN can reliably and securely exchange their information. In [34], Wyner's results were further extended to a Gaussian wiretap channel, where the notion of secrecy capacity was developed, which was obtained as the difference between the channel capacity of the main link and that of the wiretap link. If the secrecy rate is chosen below the secrecy capacity, reliable transmission from SN to DN can be achieved in perfect secrecy. In wireless networks, the secrecy capacity is severely degraded due to the time-varying fading effect of wireless channels. This is because fading attenuates the signal received at the legitimate destination, which reduces the capacity of the legitimate channel, thus resulting in a degradation of the secrecy capacity.

The family of MIMO systems is widely recognized as an effective means of mitigating the effects of wireless fading, which simultaneously increases the secrecy capacity in fading environments. In [120], Khisti et al. investigated a so-called MISOME scenario, where both the source and eavesdropper are equipped with multiple antennas, whereas the intended destination has a single antenna. Assuming that the fading coefficients of all the associated wireless channels are fixed and known to all nodes (i.e., to the source, destination and eavesdropper), the secrecy capacity of the MISOME scenario can be characterized in terms of its generalized eigenvalues. Bearing in mind that the knowledge of the wiretap channel's impulse response is typically unavailable, Khisti et al. [121] advocated the employment of a socalled masked beamforming scheme [118] for enhancing wireless physical-layer security, where the eavesdropper's channel knowledge is not relied upon for determining the transmit directions. It was shown that the masked beamforming scheme is capable of achieving a near-optimal security performance at sufficiently high SNRs. Moreover, Khisti et al. extended their results to time-varying wireless channels and developed both an upper and a lower bound on the secrecy capacity of the MISOME scenario operating in Rayleigh fading environments. In a nutshell, the work of Khisti et al. [121] was mainly focused on characterizing the secrecy capacity of masked beamforming in an information-theoretic sense, which thus belongs to the family of information-theoretic security solutions. 
Table 8 Major Information-Theoretic Security Techniques

\begin{tabular}{|l|l|l|}
\hline Year & Author(s) & Contribution \\
\hline $\mathbf{1 9 4 9}$ & Shannon [119] & $\begin{array}{l}\text { introduced the notions of theoretical secrecy and practical secrecy to defend against an } \\
\text { eavesdropper with infinite or finite computing power. }\end{array}$ \\
\hline $\mathbf{1 9 7 5}$ & Wyner [33] & $\begin{array}{l}\text { investigated information-theoretic security for a discrete memoryless wiretap channel } \\
\text { consisting of one source, one destination and one eavesdropper. }\end{array}$ \\
\hline $\mathbf{1 9 7 8}$ & Leung et al. [34] & $\begin{array}{l}\text { studied multiple-input multiple-output (MIMO) wiretap channel and characterized the } \\
\text { secrecy capacity of MIMO in terms of generalized eigenvalues. }\end{array}$ \\
\hline $\mathbf{2 0 0 7}$ & Khisti et al. [121], [122] & $\begin{array}{l}\text { introduced the notion of outage secrecy capacity and derived a closed-form expression } \\
\text { of the outage secrecy capacity using the Taylor series. }\end{array}$ \\
\hline $\mathbf{2 0 0 9}$ & Chrysikos et al. [123] & $\begin{array}{l}\text { analyzed the secrecy capacity of multiple antenna systems assuming that the CSI of } \\
\text { both the main channels and wiretap channels is known. }\end{array}$ \\
\hline $\mathbf{2 0 1 1}$ & Oggier et al. [124] & $\begin{array}{l}\text { investigated a MIMO broadcast wiretap channel without knowing the knowledge of } \\
\text { the eavesdropper's CSI and proposed a GSVD based coding scheme to achieve the } \\
\text { optimal secrecy degree of freedom region. }\end{array}$ \\
\cline { 2 - 3 } & He et al. [125] & \\
\hline
\end{tabular}

As a further development, Khisti et al. [122] examined the information-theoretic security achieved with the aid of multiple antennas in a more general scenario, where the source, the destination, and the eavesdropper are assumed to have multiple antennas. They considered two cases: 1) the simplified and idealized deterministic case in which the CSIs of both the main links and of the wiretap links are fixed and known to all the nodes; and 2) the more practical fading scenario, where the wireless channels experience time-varying Rayleigh fading and the source has the main channels' perfect CSI as well as the wiretap channels' statistical CSI knowledge. For the idealized deterministic case, they proposed the employment of the GSVD-based approach for increasing the secrecy capacity in high SNR regions. The GSVD scheme's performance was then further investigated in the fading scenario and the corresponding secrecy capacity was shown to approach zero if and only if the ratio of the number of eavesdropper antennas to source antennas was larger than two. Additionally, in [123], Chrysikos et al. investigated the wireless information-theoretic security in terms of outage secrecy capacity, which is used for characterizing the maximum secrecy rate under a given outage probability requirement. A closed-form expression of the outage secrecy capacity was derived in [123] by using the first-order Taylor series for approximation of an exponential function.

The MIMO wiretap channel can also be regarded as a MIMO broadcast channel, where SN broadcasts its confidential information to both its legitimate DN and unintentionally also to an unauthorized eavesdropper. Perfect secrecy is achieved, when SN and DN can reliably communicate at a positive rate, while ensuring that the mutual information between the SN and eavesdropper becomes zero. In [124], Oggier and Hassibi analyzed the secrecy capacity of multiple-antenna-aided systems by converting the MIMO wiretap channel into a MIMO broadcast channel, where the number of antennas is arbitrary for both the transmitter and the pair of receivers (i.e., that of DN and of the eavesdropper).
It was proven that through optimizing the transmit covariance matrix, the secrecy capacity of the MIMO wiretap channel is given by the difference between the capacity of the SN-to-DN channel and that of the $\mathrm{SN}$-to-eavesdropper channel. It was pointed out that the secrecy capacity results obtained in [124] are based on the idealized simplifying assumption that SN knows the CSI of both the main channels and of the wiretap channels. This assumption is, however, invalid in practical scenarios, since the eavesdropper is passive and hence it remains an open challenge to estimate the eavesdropper's CSI. It is of substantial interest to study a more practical scenario, where SN only has statistical CSI knowledge of wiretap channels. To this end, He et al. [125] investigated a twin-receiver MIMO broadcast wiretap channel scenario, where the legitimate $\mathrm{SN}$ and $\mathrm{DN}$ are assumed to have no knowledge of the eavesdropper's CSI. A so-called "secrecy-degree-of-freedom region" was developed for wireless transmission in the presence of an eavesdropper and a GSVD-based scheme was proposed for achieving the optimal secrecy-degreeof-freedom region. The major information-theoretic security techniques are summarized in Table 8 .

\section{B. Artificial-Noise-Aided Security}

The artificial-noise-aided security allows SN to generate specific interfering signals termed as artificial noise so that only the eavesdropper is affected adversely by the interfering signals, while the intended DN remains unaffected. This results in a reduction of the wiretap channel's capacity without affecting the desired channel's capacity and thus leads to an increased secrecy capacity, which was defined as the difference between the main channel's and the wiretap channel's capacity. Hence, a security improvement is achieved by using artificial noise. In [126], Goel and Negi considered a wireless network consisting of an $\mathrm{SN}$, a DN, and an eavesdropper for investigating the benefits of the artificial noise generation paradigm. More specifically, SN allocates a certain fraction of its transmit power for producing artificial 
noise, so that only the wiretap channel condition is degraded, while the desired wireless transmission from SN to DN remains unaffected by the artificial noise. To meet this requirement, Goel and Negi [126] proposed the employment of multiple antennas for generating artificial noise and demonstrated that the number of transmit antennas at SN has to be higher than that of the eavesdropper for ensuring that the artificial noise would not degrade the desired channel. It was shown that a nonzero secrecy capacity can be guaranteed for secure wireless communications by using artificial noise, even if the eavesdropper is closer to SN than DN.

Although the artificial-noise-aided security is capable of guaranteeing the secrecy of wireless transmission, this is achieved at the cost of wasting precious transmit power resources, since again, a certain amount of transmit power has to be allocated for generating the artificial noise. In [127], Zhou an McKay further examined the optimal transmit power sharing between the informationbearing signal and the artificial noise. They analyzed secure multiple-antenna communications relying on artificial noise and derived a closed-form secrecy capacity expression for fading environments, which was used as the objective function for quantifying the optimal power sharing between the information signal and artificial noise. The simple equal-power sharing was shown to be a nearoptimal strategy, provided that the eavesdroppers do not collude with each other to jointly perform interception. Moreover, as the number of eavesdroppers increases, more power should be allocated for generating the artificial noise. In the presence of imperfect CSI, it was observed that assigning more power to the artificial noise for jamming the eavesdroppers is capable of achieving a better security performance than increasing the transmit power of the desired information signal.

However, the aforementioned artificial-noise-aided security work has been mainly focused on improving the secrecy capacity without considering the QoS requirements of the legitimate DN. Hence, in order to address this problem, a QoS-based artificial-noise-aided security approach was presented in [128] for minimizing the maximum attainable SINR encountered at the eavesdroppers, while simultaneously guaranteeing a satisfactory SINR at the intended DN. The optimization of the artificial noise distribution was formulated based on the CSIs of both the main channels and wiretap channels, which was shown to be a nondeterministic polynomial-time hard (NP-hard) problem. The classic SDR technique [128] was used for approximating the solution of this NP-hard problem. Liao et al. [128] demonstrated that the proposed QoS-based artificial-noise-aided security scheme is capable of efficiently guarding against eavesdropping attacks, especially in the presence of a large number of eavesdroppers. Li and Ma [129] proposed a robust artificial-noise-aided security scheme for a MISOME wiretap channel. Assuming that $\mathrm{SN}$ has perfect CSI knowledge of the main channels, but imperfect CSI knowledge of the wiretap channels, an optimization problem was formulated for the secrecy rate maximization with respect to both the desired signal's and the artificial noise's covariance, which is a semi-infinite optimization problem and can be solved with the aid of a simple 1-D search algorithm. It was shown that the proposed robust artificial noise design significantly outperforms conventional nonrobust approaches in terms of its secrecy capacity.

In addition to relying on multiple antennas for artificial noise generation, cooperative relays may also be utilized for producing artificial noise to guard against eavesdropping attacks. In [130], Goeckel et al. studied the employment of cooperative relays for artificial noise generation and proposed a secret wireless communications protocol, where a messaging relay was used for assisting the legitimate transmissions from SN to DN and a set of intervening relays were employed for generating the artificial noise invoked for jamming the eavesdroppers. The main focus of [130] was to quantify how many eavesdroppers can be tolerated without affecting the communications secrecy in a wireless network supporting a certain number of legitimate nodes. It was shown that if the eavesdroppers are uniformly distributed and their locations are unknown to the legitimate nodes, the tolerable number of eavesdroppers increases linearly with the number of legitimate nodes. The major artificialnoise-aided security techniques are summarized in Table 9.

\section{Security-Oriented Beamforming Techniques}

The family of security-oriented beamforming techniques allows SN to transmit its information signal in a particular direction to the legitimate DN, so that the signal received at an eavesdropper (that typically lies in a direction different from DN) experiences destructive interference and hence it becomes weak. Thus, the RSS of DN would become much higher than that of the eavesdropper with the aid of security-oriented beamforming, leading to a beneficial secrecy capacity enhancement. In [131], Zhang and Gursoy proposed the employment of cooperative relays to form a beamforming system relying on the idealized simplifying assumption of having the perfect CSI knowledge of all the main channels as well as of the wiretap channels and conceived a decode-andforward-relay-based beamforming design for maximizing the secrecy rate under a fixed total transmit power constraint. The formulated problem was then solved by using the classic semidefinite programming and secondorder cone programming techniques. It was shown in [131] that the proposed beamforming approach is capable of significantly increasing the secrecy capacity of wireless transmissions.

In [132], multiple antennas were used for beamforming in order to improve the attainable secrecy capacity of 
Table 9 Major Artificial-Noise-Aided Security Techniques

\begin{tabular}{|c|l|l|}
\hline Year & Author(s) & Contribution \\
\hline \hline $\mathbf{2 0 0 8}$ & Goel et al. $[126]$ & $\begin{array}{l}\text { proposed the use of multiple antennas to generate artificial noise and showed that } \\
\text { a non-zero secrecy capacity for secure communications can be guaranteed. }\end{array}$ \\
\hline $\mathbf{2 0 1 0}$ & Zhou et al. [127] & $\begin{array}{l}\text { examined the optimal transmit power allocation between the information-bearing } \\
\text { signal and the artificial noise. }\end{array}$ \\
\hline $\mathbf{2 0 1 1}$ & Liao et al. $[128]$ & $\begin{array}{l}\text { proposed a QoS based artificial noise aided security approach to minimize the } \\
\text { maximum SINR received at eavesdroppers while guaranteeing a satisfactory SINR } \\
\text { received at the intended destination. }\end{array}$ \\
\cline { 2 - 4 } & Li et al. $[129]$ & $\begin{array}{l}\text { studied a secrecy rate maximization problem with respective to the desired signal } \\
\text { covariance and the artificial noise covariance for a MISOME wiretap channnel. }\end{array}$ \\
\cline { 2 - 4 } & Goeckel et al. [130] & $\begin{array}{l}\text { investigated the use of cooperative relays for the artificial noise generation and } \\
\text { proposed a relay based secret wireless communications protocol. }\end{array}$ \\
\hline
\end{tabular}

wireless transmissions from $\mathrm{SN}$ to $\mathrm{DN}$ in the presence of an eavesdropper. In contrast to the work presented in [131], where the perfect CSI knowledge of the wiretap channel was assumed, Mukherjee and Swindlehurst conceived the optimal beamforming designs in [132] without relying on the idealized simplifying assumption of knowing the eavesdropper's CSI, albeit the exact CSI of the main channel spanning from SN to DN was still assumed to be available. However, the perfect CSI of the main channel is typically unavailable at SN. To this end, Mukherjee and Swindlehurst further studied the impact of imperfect CSI on the attainable physical-layer security performance and presented a pair of robust beamforming schemes that are capable of mitigating the effect of channel estimation errors. It was shown that the proposed robust beamforming techniques perform well for moderate CSI estimation errors and hence achieve a higher secrecy capacity than the artificial-noise-aided security approaches.

In addition, Jeong et al. [133] investigated the benefits of transmit beamforming in an amplify-and-forward relay network consisting of an SN, an RN, and a DN, where the $\mathrm{RN}$ is indeed potentially capable of improving the SN-to-DN link, but it is also capable of launching a passive eavesdropping attack. Hence, a pair of secure beamforming schemes, namely a noncooperative beamformer and a cooperative secure beamformer, were proposed for maximizing the secrecy capacity of the SN-to-DN link. Extensive simulation results were provided for demonstrating that the secure beamforming schemes proposed are capable of outperforming conventional security approaches in terms of the attainable secrecy capacity. Moreover, in [134], a cross-layer approach exploiting the multiple simultaneous data streams of the family of operational IEEE 802.11 standards was devised by using zeroforcing beamforming, where a multiantenna-assisted AP was configured to utilize one of its data streams for communicating with the desired user, while the remaining data streams were exploited for actively interfering with the potential eavesdroppers. Extensive experimental evaluations were carried out in practical indoor WLAN environments, demonstrating that the proposed zero-forcing beamforming method consistently granted an SINR for the desired user, which was $15 \mathrm{~dB}$ higher than that of the eavesdropper.

Naturally, this beamforming technique may also be combined with the artificial-noise-based approach for the sake of further enhancing the physical-layer security of wireless transmissions against eavesdropping attacks. Hence, in [135], Qin et al. examined a joint beamforming and artificial-noise-aided design for conceiving secure wireless communications from $\mathrm{SN}$ to $\mathrm{DN}$ in the presence of multiple eavesdroppers. The beamforming weights and artificial noise covariance were jointly optimized by minimizing the total transmit power under a specific target secrecy rate constraint. To elaborate a little further, this joint beamforming and artificial-noise-aided design problem was solved by using a two-level optimization approach, where the classic semidefinite relaxation method and the golden-section-based method [135] were invoked for the inner-level optimization and the outer-level optimization, respectively. Numerical results illustrated that the joint beamforming and artificial-noise-aided scheme significantly improves the attainable secrecy capacity of wireless transmission as compared to the conventional security-oriented beamforming approaches. In [136], Romero-Zurita et al. studied the joint employment of spatial beamforming and artificial noise generation for enhancing the attainable physical-layer security of a MISO channel in the presence of multiple eavesdroppers, where no CSI knowledge was assumed for the wiretap channel. The optimal power sharing between the information signal and artificial noise was examined under a specific guaranteed secrecy probability requirement. By combining the beamforming and artificial noise techniques, both the security and reliability of wireless transmissions were substantially improved. The major security-oriented beamforming techniques are summarized in Table 10.

\section{Diversity-Assisted Security Approaches}

This section is focused on the portrayal of diversity techniques invoked for the sake of improving the physical-layer security of wireless transmissions [137]. In contrast to the artificial-noise-aided approaches, which 
Table 10 Major Security-Oriented Beamforming Techniques

\begin{tabular}{|c|l|l|}
\hline Year & Author(s) & Contribution \\
\hline $\mathbf{2 0 1 0}$ & Zhang et al. [131] & $\begin{array}{l}\text { proposed a cooperative relay based beamforming scheme to maximize the secrecy rate } \\
\text { with the perfect CSI of the main channels and wiretap channels. }\end{array}$ \\
\hline $\mathbf{2 0 1 1}$ & Mukherjee et al. $[132]$ & $\begin{array}{l}\text { studied the optimal beamforming design in MIMO wiretap channels without knowing } \\
\text { the eavesdropper's CSI knowledge. }\end{array}$ \\
\hline $\mathbf{2 0 1 2}$ & Jeong et al. [133] & $\begin{array}{l}\text { investigated the transmit beamforming in untrusted relay networks and proposed the } \\
\text { noncooperative secure beamforming and cooperative secure beamforming schemes. }\end{array}$ \\
\cline { 2 - 3 } & Romero-Zurita et al. [136] & $\begin{array}{l}\text { proposed a joint spatial beamforming and artificial noise scheme for physical-layer } \\
\text { security improvement. }\end{array}$ \\
\hline
\end{tabular}

dissipate additional power assigned to the artificial noise generation, the diversity-aided security paradigm is capable of enhancing the wireless security without any additional power. Traditionally, diversity techniques have been used for improving the attainable transmission reliability, but they also have a substantial potential in terms of enhancing the wireless security against eavesdropping attacks. Below we will discuss several diversity-aided security approaches, including multiple-antenna-aided diversity, multiuser diversity, and cooperative diversity.

Multiple-antenna-aided transmit diversity has been shown to constitute an effective means of combatting the fading effect, hence also increasing the secrecy capacity of wireless transmissions [138], [139]. As shown in Fig. 19, provided that SN has multiple antennas, the optimal antenna can be activated for transmitting the desired signal, depending on whether the CSI of the main channel and of the wiretap channel is available. To be specific, if the CSI of both the main channel and of the wiretap channel is known at $\mathrm{SN}$, the specific transmit

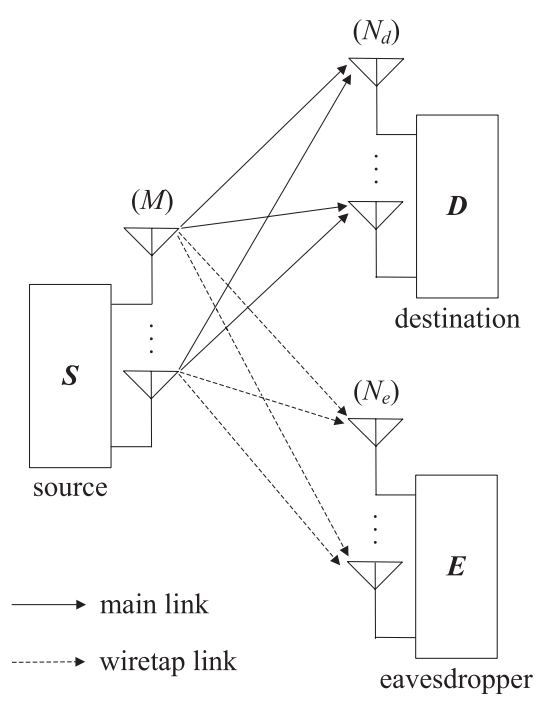

Fig. 19. MIMO wireless system consisting of an SN and a DN in the presence of an eavesdropper, where $M, N_{d}$, and $N_{e}$ represent the number of antennas at SN, DN, and eavesdropper, respectively. antenna associated with the highest secrecy capacity can be chosen as the optimal antenna to transmit the desired signal, which has the potential of significantly improving the secrecy capacity of wireless transmissions. If only the main channel's CSI is available, we can choose a transmit antenna associated with the highest main channel capacity to transmit the desired signal. Since the transmit antenna selection is exclusively based on the main channel's CSI and the wiretap channel is typically independent of the main channel, the main channel's capacity will be increased with the aid of transmit antenna selection, while no capacity improvement can be achieved for the wiretap channel. This finally results in an increase of the secrecy capacity, as an explicit benefit of transmit antenna selection.

The multiuser diversity of Fig. 20 also constitutes an effective means of improving the physical-layer security in the face of eavesdropping attacks. Considering that a BS serves multiple users in a cellular network, an orthogonal multiple access mechanism, such as the OFDMA of LTE [140] or CDMA of 3G systems [141], enables the multiple users to communicate with the BS. Considering the OFDMA as an example, given a slot or subband of OFDM subcarriers, we should determine which particular user is assigned to access this specific subband for data transmission. More specifically, a user is enabled with the aid of multiuser scheduling to access the OFDM

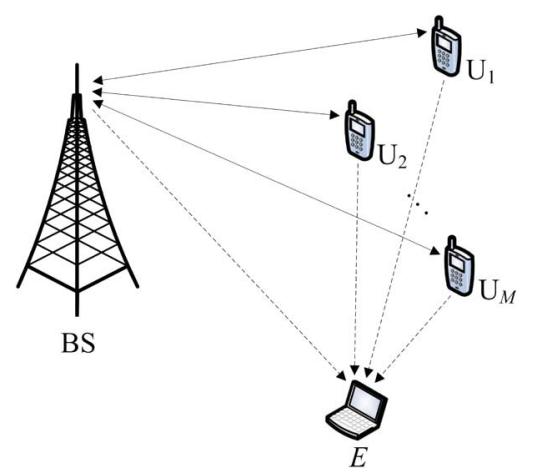

Fig. 20. Multiuser diversity system consisting of a BS and $M$ users in the presence of an eavesdropper. 


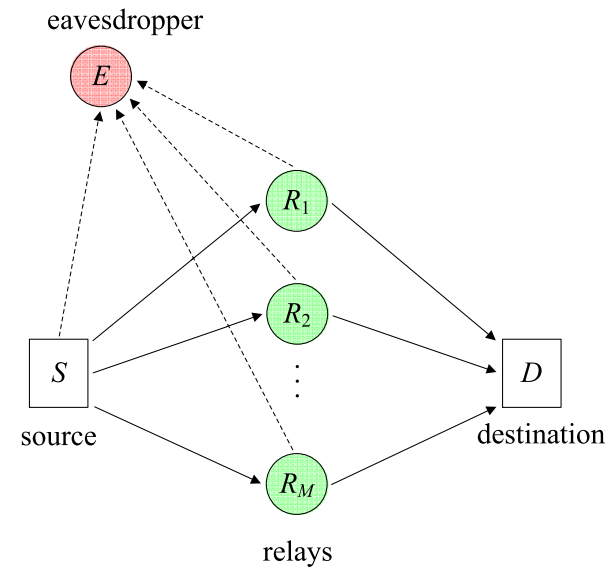

Fig. 21. Cooperative diversity system consisting of an SN, $M$ relays, and a DN in the presence of an eavesdropper.

subband and then starts transmitting its signal to the BS. Meanwhile, due to the broadcast nature of wireless medium, an eavesdropper may intercept the source message. In order to effectively protect the wireless transmission against eavesdropping attacks, the multiuser scheduling should be designed for minimizing the capacity of the wiretap channel, while maximizing the capacity of the main channel [142]. This action requires the CSI of both the main channel and of the wiretap channel. If only the main channel's CSI is available, the multiuser scheduling can be designed for maximizing the main channel's capacity without the wiretap channel's CSI knowledge. It is worth mentioning that the multiuser scheduling is capable of significantly improving the main channel's capacity, while the wiretap channel's capacity remains the same, which results in a secrecy capacity improvement with the aid of multiuser diversity, even if the CSI of the wiretap channel is unknown.

As an alternative, cooperative diversity [143], [144] also has a great potential in terms of protecting the wireless transmissions against eavesdropping attacks. When considering a wireless network consisting of a single SN, multiple RNs, and a DN as shown in Fig. 21, the multiple relays can be exploited for assisting the signal transmission from $\mathrm{SN}$ to $\mathrm{DN}$. In order to prevent the eavesdropper from intercepting the source signal from a security perspective, the best relay selection emerges as a means of improving the security of wireless transmissions against eavesdropping attacks [145]. Specifically, an RN having the highest secrecy capacity (or the highest main channel capacity if only the main channel's CSI is known) is selected to assist the SN's transmission to the intended DN. By using the best RN selection, a beneficial cooperative diversity gain can be achieved for the sake of increasing the secrecy capacity, which explicitly demonstrates the advantages of wireless physical-layer security.

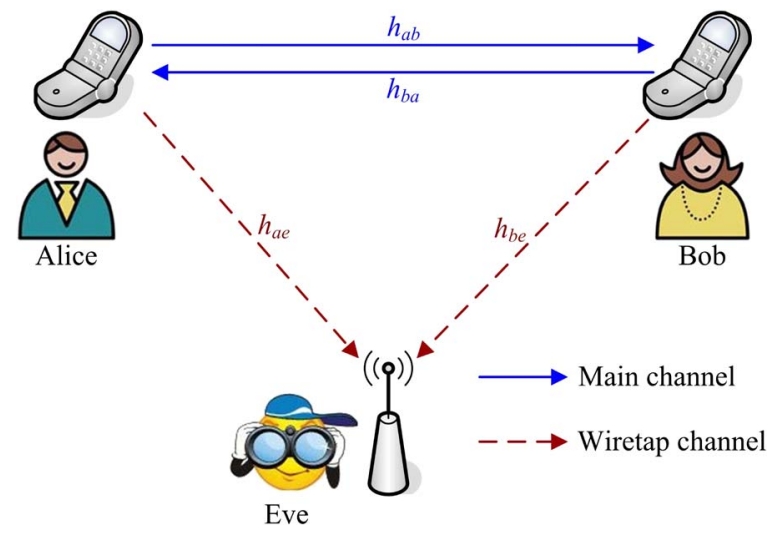

Fig. 22. Wireless system consisting of two legitimate transceivers (Alice and Bob) in the presence of an eavesdropper (Eve).

\section{E. Physical-Layer Secret Key Generation}

In this section, we present the family of wireless secret key generation techniques by exploiting the physicallayer characteristics of radio propagation, including the amplitude and phase of wireless fading [146]. To be specific, as shown in Fig. 22, a pair of legitimate transceivers, namely Alice and Bob are connected through a reciprocal wireless channel, where the fading gain of the main channel spanning from Alice to Bob, denoted by $h_{a b}$, is identical to that from Bob to Alice, namely $h_{b a}$. Since Alice and Bob can directly estimate $h_{b a}$ and $h_{a b}$, respectively, using classic channel estimation methods [147], [148], they may exploit their estimated CSIs $\hat{h}_{b a}$ and $\hat{h}_{a b}$ for the secret key generation and agreement process. By contrast, a third party (e.g., Eve) based at a different location experiences independent wiretap channels of $h_{a e}$ and $h_{b e}$, which are uncorrelated with the CSIs $h_{a b}$ and $h_{b a}$ of the main legitimate channel between Alice and Bob, as seen in Fig. 22. Since Alice and Bob both estimate the main channel by themselves without exchanging their estimated CSIs $\hat{h}_{a b}$ and $\hat{h}_{b a}$ over the air, it is impossible for Eve to acquire the main channel's CSI for deriving and duplicating the secret keys. The secret key extraction and agreement process based on the physical characteristics of the main channel is capable of achieving reliable information-theoretic security without resorting to a fixed key management infrastructure [149].

The research of physical-layer key generation and agreement can be traced back to the middle of the 1990s [150], [151], where the feasibility of generating secret keys based on the wireless channel's CSI was shown to achieve reliable information-theoretic security without devising any practical key extraction algorithms. To this end, an RSS-based secret key extraction algorithm was proposed in [152] by exploiting RSS measurements of the main channel in order to generate secret bits for an IEEE 802.11 network in an indoor wireless environment. In [153], Jana et al. further investigated the key 
generation rate of RSS-based secret key extraction in diverse wireless environments. It was shown in [153] that it is possible to generate secret bits at a sufficiently high rate based on the wireless channel variations in highly dynamic mobile scenarios. However, in static environments, where the network devices are fixed, the rate of bits generated is too low to be suitable for a secret key, which is due to the lack of random variations in the wireless channels.

To this end, Gollakota and Katabi [154] proposed the so-called iJam approach, which processed the desired transmit signal in a specific manner that still allows the legitimate receiver to decode the desired signal, but prevents the potential eavesdroppers from decoding it. The iJam scheme renders the secret key generation both fast and independent of the wireless channel variations. Furthermore, a testbed was also developed in [154] for implementing the iJam technique using USRP2 radios and the IEEE 802.11 specifications. The associated experimental results demonstrated that the iJam scheme was indeed capable of generating the physical-layer secret keys faster than conventional approaches. To be specific, the iJam scheme generated secret keys at a rate of $3-18 \mathrm{~kb} / \mathrm{s}$ without any measurable disagreement probability, whereas the conventional approaches exhibited a maximum generation rate of $44 \mathrm{~b} / \mathrm{s}$ in conjunction with a $4 \%$ bit disagreement probability between the legitimate transmitter and receiver. More recently, an extension of the RSS-based key extraction from a twin-device system to a multidevice network was studied in [155], where a collaborative key generation scheme was proposed for multiple devices by exploiting the RSS measurements and then experimentally validating it in both indoor and outdoor environments.

Although it is feasible to exploit the RSS for wireless secret key extraction and agreement, the RSS-based methods have a low key generation rate, which limits their applications in practical wireless systems. In order to alleviate this problem, the channel phase may also be considered as an alternative means of assisting the generation of secret keys, which is capable of beneficially exploiting the phase measurements across different carriers and thus enhances the secret key generation rate. In [156], Shehadeh et al. proposed a channel-phase-based key agreement scheme, which generates secret bits from the time-varying frequency-domain characteristics in an OFDM-based wireless system. More specifically, the OFDM system's subcarrier phase correction process was studied in the context of secret key generation, showing that the employment of higher FFT sizes is potentially capable of improving the secret bit generation rate. Additionally, Wang et al. [157] employed multiple randomized channel phases for conceiving an efficient key generation scheme, which was evaluated through both analytical and simulation studies. This solution was found to be highly flexible in the context of multiuser wireless networks and increased the secret key generation rate by orders of magnitude. It has to be pointed out that exploiting the phase measurements across multiple OFDM subcarriers is beneficial in terms of increasing the attainable key generation rate. However, the channel phase extracted by a pair of legitimate devices is unlikely to be reciprocal due to the different hardware characteristics of the different devices. This nonreciprocity embedded in the phase measurements results in a high disagreement rate for the legitimate devices during the generation of secret keys.

As an alternative, MIMO techniques used by the legitimate transceivers are capable of significantly increasing the channel's randomness, which can be exploited for secret key generation and agreement, leading to the concept of MIMO-based key generation. In [158], a theoretical characterization of the MIMO-based key generation was explored in terms of deriving a performance limit on the number of secret key bits generated per random channel realization, assuming that the main channel and the wiretap channel are Rayleigh distributed. As a further development, Zeng et al. proposed a practical multiple-antenna-based secret key generation protocol in [159], which was implemented for an IEEE 802.11 network in both indoor and outdoor mobile environments. It was also shown in [160] that even if an eavesdropper is capable of increasing the number of its antennas, it cannot infer more information about the secret keys generated from the main channel. However, the secrecy improvement of MIMO-based secret key generation is achieved at the cost of an increased system complexity, since more computing and memory resources are required for estimating the MIMO channel, as the number of transmit/receive antennas increases. In order to further improve the reliability and efficiency of secret key generation, the employment of relay nodes was investigated in [160] for assisting the secret key generation in two different scenarios, namely in conjunction with a single-antenna-aided relay and a multiple-antenna assisted relay, respectively. It was demonstrated in [160] that the relay-channel-based key generation method is capable of substantially improving the key generation rate in Rayleigh fading environments. Although the relay nodes can be exploited for enhancing the key generation rate, they may become compromised by an adversary aiming for launching malicious activities. Hence, it is of interest to explore the security issues associated with untrusted relays as well as the corresponding countermeasures.

It is worth mentioning that the success of the aforementioned physical-layer key generation solutions relies on the assumption that the main channel between the transmitter and the legitimate receiver is reciprocal and uncorrelated with the wiretap channel experienced at an eavesdropper located more than half-a-wavelength away from the legitimate receiver. However, this assumption 
Table 11 Major Physical-Layer Secret Key Generation Techniques

\begin{tabular}{|c|l|l|}
\hline Year & Author(s) & Contribution \\
\hline \hline $\mathbf{1 9 9 5}$ & Hershey and Hassan et al. [150] & $\begin{array}{l}\text { proved the feasibility of generating secret keys from wireless channels for achieving } \\
\text { information-theoretical security. }\end{array}$ \\
\hline $\mathbf{2 0 0 8}$ & Mathur et al. [152] & $\begin{array}{l}\text { proposed the use of RSS measurements of the legitimate wireless channel to generate } \\
\text { secret key bits. }\end{array}$ \\
\hline $\mathbf{2 0 0 9}$ & Wallace et al. [158] & $\begin{array}{l}\text { derived a theoretical limit on the secret key generation rate for MIMO wireless com- } \\
\text { munications system. }\end{array}$ \\
\hline $\mathbf{2 0 1 0}$ & Zeng et al. [159] & $\begin{array}{l}\text { proposed a practical MIMO channel based secret key generation protocol along with } \\
\text { its implementation in a real IEEE } 802.11 \text { network. }\end{array}$ \\
\hline $\mathbf{2 0 1 1}$ & $\begin{array}{l}\text { Shehadeh and Wang } \text { et al. } \\
\text { [156], [157] }\end{array}$ & $\begin{array}{l}\text { explored the channel phase based key generation and agreement by using time-varying } \\
\text { frequency characteristics in OFDM wireless systems }\end{array}$ \\
\cline { 2 - 3 } & Shimizu et al. [160] & $\begin{array}{l}\text { studied the employment of relay nodes to assist the secret key generation and showed } \\
\text { the effectiveness of relay channel based key extraction In fading environments. }\end{array}$ \\
\hline $\mathbf{2 0 1 3}$ & Shi et al. [163] & $\begin{array}{l}\text { proposed an authenticated secret key generation scheme for achieving the simul- } \\
\text { taneous device authentication and secret key extraction. }\end{array}$ \\
\hline
\end{tabular}

has not been rigorously evaluated in the open literature and indeed, it maybe invalid in some practical scenarios, which do not experience extensive multipath scattering. It was shown in [161] that in reality a strong correlation may be encountered between the main channel and the wiretap channel, even when the eavesdropper is located significantly more than half-a-wavelength away from the legitimate receiver. In [161], Edman et al. demonstrated that a so-called passive inference attacker is potentially capable of exploiting this correlation for inferring a part of the secret keys extracted between a pair of legitimate devices. Additionally, in [162], Eberz et al. presented a practical MITM attack against the physical-layer key generation and showed that the MITM attack can be readily launched by impersonating the legitimate transmitter and receiver as well as by injecting the eavesdropper's data packets. It was demonstrated in [162] that the MITM attack is capable of imposing intentional sabotaging of the physical-layer key generation by inflicting a high key disagreement rate, while additionally inferring up to $47 \%$ of the secret keys generated between the legitimate devices.

In order to mitigate the effects of MITM attacks, Shi et al. [163] examined the potential benefits of simultaneous device authentication and secret key extraction based on the wireless physical-layer characteristics, where an ASK scheme was proposed by exploiting the heterogeneous channel characteristics in the context of wireless body area networks. Specifically, in case of simple routine body movements, the variations of wireless channels between line-of-sight on-body devices are relatively insignificant, while the wireless channels between the non-line-of-sight devices fluctuate quiet significantly. The ASK scheme exploits the relatively static channels for reliable device authentication and the dynamically fluctuating channels for secret key generation. Extensive experiments were conducted by using lowend commercial-off-the-shelf sensors, demonstrating that the ASK scheme is capable of effectively authenticating body devices, while simultaneously generating secret keys at a high rate. More importantly, the ASK is resilient to MITM attacks, since it performs the authentication and key generation simultaneously. Consequently, it becomes difficult for an MITM attacker to promptly pass through the authentication phase and to get involved in the resultant key generation phase. The major physical-layer secret key generation techniques are summarized in Table 11 at a glance.

\section{WIRELESS JAMMING ATTACKS AND THEIR COUNTERMEASURES}

As mentioned earlier, due to the shared nature of radio propagation, wireless transmissions are vulnerable to both the eavesdropping and jamming attacks. In Section V, we have presented a comprehensive overview of how physical-layer security may be exploited for guarding against eavesdropping. Let us now focus our attention on the family of wireless jamming attacks and their countermeasures in this section. In wireless networks, a jamming attack can be simply launched by emitting unwanted radio signals to disrupt the transmissions between a pair of legitimate nodes.

The objective of a jamming attacker (also referred to as jammer) is to interfere with either the transmission or the reception (or both) of legitimate wireless communications. For example, a jammer may continuously transmit its signal over a shared wireless channel so that legitimate nodes always find the channel busy and keep deferring their data transmissions. This, however, is energy-inefficient, since the jammer has to transmit constantly. To improve its energy efficiency, a jammer may opt for transmitting an interfering signal only when it detects that a legitimate transmitter is sending data. There are many different types of wireless jammers, which may be classified into the following five categories [164]:

1) constant jammer, where a jamming signal is continuously transmitted; 
2) intermittent jammer, where a jamming signal is emitted from time to time;

3) reactive jammer, where a jamming signal is only imposed, when the legitimate transmission is detected to be active;

4) adaptive jammer, where a jamming signal is tailored to the level of received power at the legitimate receiver;

5) intelligent jammer, where weaknesses of the upper-layer protocols are exploited for blocking the legitimate transmission.

Clearly, the first four types of jammers all exploit the shared nature of the wireless medium and can be regarded as wireless physical-layer jamming attacks. By contrast, the intelligent jammer attempts to capitalize on the vulnerabilities of the upper-layer protocols [165], including the MAC, network, transport, and application layers. Typically, the network, transport, and application layers are defined in the TCP/IP protocols and not specified in wireless standards (e.g., Bluetooth, WLAN, etc.), which are responsible for the PHY and MAC specifications only. The jammers targeting the network, transport and application layers essentially constitute DoS attacks (e.g., Smurf attack, TCP/UDP flooding, malware attack, etc.), which have been summarized in Section III-C-E. Let us now discuss the aforementioned five main types of wireless jamming attacks and their countermeasures in a little more detail.

\section{A. Constant Jammer}

Again, the constant jammer continuously transmits a jamming signal over the shared wireless medium. The jamming signal can have an arbitrary waveform associated with a limited bandwidth and constrained power, including but not limited to pseudorandom noise, modulated Gaussian waveforms, or any other signals. The effect of a constant jammer is twofold. On the one hand, it increases the interference and noise level for the sake of degrading the signal reception quality at a legitimate receiver. On the other hand, it also makes a legitimate transmitter always find the wireless channel busy, which keeps preventing the legitimate transmitter from gaining access to the channel. Hence, the constant jammer is capable of disrupting the legitimate communications, regardless of the specific wireless system. However, the constant jammer is energy inefficient, since it has to continuously transmit a jamming signal.

The basic idea behind detecting the presence of a constant jammer is to identify an abnormal signal received at a legitimate receiver [164], [166]. There are certain statistical tests that can be exploited for the detection of the constant jammer, such as the RSS, CST, PER, etc. To be specific, the RSS test is based on a natural measurement used for detecting the presence of a constant jammer, since the signal strength received at a legitimate node would be directly affected by the presence of a jamming signal. The RSS detector first accumulates the energy of the signal received during an observation time period and then compares the accumulated energy to a predefined threshold to decide as to whether a constant jammer is present or absent. If the accumulated energy is higher than the threshold, implying that a jamming signal may be present, then the presence of a constant jammer is confirmed. As an alternative, the CST can also be used as a measurement for deciding whether a constant jammer is preventing the legitimate transmission, since the CST distribution will be affected by the jammer. More specifically, the presence of a jamming signal may render the wireless channel constantly busy and hence might lead to an unusually high CST, which can be used for jammer detection.

Additionally, the PER is defined as the number of unsuccessfully decoded data packets divided by the total number of received packets, which can also be used for detecting the presence of a jamming signal, since the legitimate communications will be severely corrupted by the constant jammer, leading to an unduly high PER. Normally, the legitimate wireless communications links operating in the absence of a jammer should have a relatively low PER (e.g., lower than 0.1). Indeed, it was shown in [166] that even in a highly congested network, the PER is unlikely to exceed 0.2. By contrast, in the presence of an effective jammer, the legitimate data transmissions will be overwhelmed by the jamming signal and background noise. This would result in an excessive PER, close to one [166], which indicates that indeed, the PER may be deemed to be an effective measurement for detecting the presence of a constant jammer. Conversely, an ineffective jammer, which only slightly affects the PER, fails to inflict a significant damage upon the legitimate wireless system and thus may not have to be detected for invoking further countermeasures.

Once the presence of a jammer is detected, it is necessary to decide upon how to defend the legitimate transmissions against jamming attacks. Frequency hopping is a well-known classic anti-jamming technique [167][169], which rapidly changes the carrier frequency with the aid of a pseudorandom sequence known to both the transmitter and the receiver. The frequency hopping regime can be either proactive or reactive. In proactive frequency hopping, the transmitter will proactively perform pseudorandom channel switching, regardless of the presence or absence of the jammer. Hence, proactive hopping does not have to detect the presence of a jammer. By contrast, reactive frequency hopping starts switching to a different channel only when the presence of a jamming signal is detected. Compared to proactive hopping, reactive hopping has the advantage of requiring a reduced number of frequency hops for achieving a certain level of secrecy. Overall, frequency hopping is highly 
resistant to jamming attacks, unless of course the jammer has explicit knowledge of the pseudorandom hopping pattern. Typically, cryptographic techniques are used for generating the pseudorandom hopping pattern under the control of a secret key that is preshared by the legitimate transmitter and receiver.

\section{B. Intermittent Jammer}

An attacker, which transmits a jamming signal from time to time for the sake of interfering with the legitimate communications, is referred to as an intermittent jammer [170]. The intermittent jammer transmits for a certain time and then sleeps for the remaining time. Typically, increasing the sleeping time would save more energy for the jammer, which of course comes at the cost of a performance degradation in terms of the jamming effectiveness, since less time becomes available for transmitting the jamming signal. The jammer can strike a tradeoff between the jamming effectiveness and energy savings by appropriately adjusting the transmit time and sleeping time. Hence, compared to the constant jammer, the intermittent jammer generally reduces the energy consumption, which is attractive for energy-constrained jammers.

Similarly to the constant jammer, the presence of an intermittent jammer will affect the same statistical measurements of the legitimate transmissions, including the RSS, CST, and PER, which thus can be used for its detection. After detecting an intermittent jammer, again frequency hopping may be activated for protecting the legitimate transmissions. More specifically, when a legitimate node is deemed to be jammed, it switches to another channel and communicates with its destination over the newly established link.

\section{Reactive Jammer}

The reactive jammer starts to transmit its jamming signal only when it detects that the legitimate node is transmitting data packets [171], [172]. This type of jammer first senses the wireless channel and upon detecting that the channel is busy, implying that the legitimate user is active, it transmits a jamming signal for the sake of corrupting the data reception at the legitimate receiver. The success of a reactive jammer depends on its sensing accuracy concerning the legitimate user's status. For example, when the legitimate signal received at a reactive jammer is weak (e.g., due to fast fading and shadowing effects) and hence cannot be detected, the reactive jammer then becomes ineffective in jamming the legitimate transmissions. In contrast to both constant and intermittent jammers that attempt to block the wireless channel regardless of the legitimate traffic activity on the channel, the reactive jammer remains quiet when the channel is idle and starts emitting its jamming signal only when the channel is deemed to be busy. This implies that the reactive jammer is more energy efficient than both the constant and intermittent jammers.

The detection of the presence of a reactive jammer is typically harder than that of the constant and intermittent jammers. As discussed above, the constant and intermittent jammers both intend to interfere with the reception of a legitimate data packet as well as to hinder the transmission of the legitimate packets by maliciously seizing the wireless channel. By contrast, a reactive jammer inflicts less damage, since it corrupts the reception without affecting the legitimate transmitter's activity to gain access to the wireless channel. This means that the CST becomes an ineffective measurement for detecting the reactive jammer. Since the reception of legitimate wireless communications will be affected in the presence of a reactive jammer, we can still consider the employment of RSS- and PER-based techniques for the detection of the reactive jammer. Generally, an abnormal increase of the RSS and/or a surprisingly high PER indicate the presence of a reactive jammer.

An effective technique of preventing a reactive jammer from disrupting communications is to assist the legitimate user in becoming undetectable, because then the jammer remains silent. DSSS [173] techniques spread the radio signal over a very wide frequency bandwidth, so that the signal has a low PSD, which may even be below the background noise level. This makes it difficult for a reactive jammer to differentiate the DSSS modulated legitimate signal from the background noise. In this way, the reactive jammer may become unable to track the legitimate traffic activity and thus cannot disrupt the legitimate transmissions. Additionally, the aforementioned frequency hopping technique is also effective in guarding against a reactive jamming attack, as long as the hopping rate is sufficiently high (e.g., faster than the jammer reacts).

\section{Adaptive Jammer}

The adaptive jammer refers to an attacker who can adjust its jamming power to any specific level required for disrupting the legitimate receiver [174]. More specifically, in wireless communication systems, the RSS depends on the time-varying fading. If the main channel spanning from the transmitter to the legitimate receiver is relatively good and the signal arriving at the legitimate receiver is sufficiently strong, the adaptive jammer may have to increase its jamming power for the sake of corrupting the legitimate reception. One the other hand, if the main channel itself experiences an outage due to a deep fade, then naturally, the legitimate receiver is unable to succeed in decoding its received signal even in the absence of a jammer. In this extreme case, no jamming power is needed for the adaptive jammer. Hence, compared to the constant, intermittent, and reactive jammers, the adaptive jammer is the most energy-efficient jamming attacker, which can achieve the highest energy 
efficiency when aiming for disrupting the legitimate transmissions. It can be observed that the adaptive jammer should have the RSS knowledge of the legitimate receiver for adapting its jamming power, which, however, is challenging for a jammer to obtain in practice, since the main channel's RSS varies in time and it is unknown to the jammer. This limits the application of the adaptive jammer in practical wireless systems. The adaptive jammer usually serves as an idealized optimum jamming attacker for benchmarking purposes.

The detection of an adaptive jammer is challenging in the sense that it will dynamically adjust its jamming power to conceal its existence. Similarly to the reactive jammer, the adaptive jammer transmits nothing if the legitimate transmission is deemed to be inactive, implying that the CST technique is ineffective for detecting the adaptive jammer. Although the RSS- and PER-based solutions can be employed for detecting the presence or absence of an adaptive jammer, the separate employment of the two individual statistics may be insufficient. As a consequence, $\mathrm{Xu}$ et al. proposed a so-called consistency check method in [166], which relies on the joint use of the RSS and PER measurements. To be specific, if both the RSS and the PER are unexpectedly high, it is most likely that there is a jammer, which results in a high RSS due to the presence of a jamming signal that interferes with the legitimate reception, leading to a high PER. If we encounter a low RSS and a high PER, this implies that the main channel is poor. Moreover, the joint occurrence case of a high RSS and a low PER indicates that the legitimate transmissions perform well. Finally, it is unlikely in general that both the RSS and PER are low simultaneously.

As mentioned above, the adaptive jammer is an idealized adversary who is assumed to have knowledge of the legitimate signal characteristics, including the RSS, carrier frequency and bandwidth, waveform, and so on. In order to guard against such a sophisticated jamming attacker, a simple but effective defense strategy is to evade the adversary. Hence, in [166], Xu et al. proposed a pair of evasion methods to defend against jamming attacks, namely the channel surfing and the spatial retreating solutions. To be specific, in channel surfing, the legitimate transmitter and receiver are allowed to change their jammed channel to a new channel operating at the link layer, which is a different philosophy from that of frequency hopping operating at the physical layer. The spatial retreating technique enables a jammed wireless node to move away and escape from the jammed area to avoid the jamming signal. In case of spatial retreating, it is crucial to accurately determine the position of jammers, which enables the victims to move away from the jammed area. To this end, in [175], Liu et al. proposed an error-minimizing framework for accurately localizing multiple jammers by relying on a direct measurement of the jamming signal strength, demonstrating its advantage

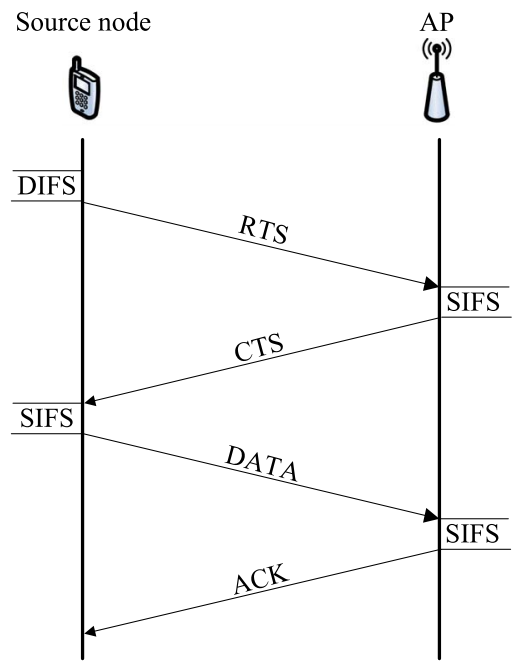

Fig. 23. IEEE 802.11 DCF process.

over conventional methods in terms of its localization accuracy.

\section{E. Intelligent Jammer}

The jamming attackers discussed so far belong to the family of physical-layer jammers operating without taking into account any upper-layer protocol specifications. By contrast, an intelligent jammer is assumed to have a good understanding of the upper-layer protocols and attempts to jam the vitally critical network control packets (rather than data packets) by exploiting the associated protocol vulnerabilities. This section is mainly focused on the jamming of MAC control packets. For example, let us consider the MAC jamming of the IEEE 802.11 standard (also known as Wi-Fi) that is widely used for WLANs [176]. The IEEE 802.11 MAC procedure is referred to as the DCF [177], which is shown in Fig. 23. To be specific, if a source node senses the channel to be idle, it waits for a time period termed as the DIFS and then sends an RTS control frame to an AP. After succeeding in decoding the RTS frame and waiting for a time period called the SIFS, the AP will send a CTS control frame, which indicates that the AP is ready to receive data packets. Finally, the source node waits for a SIFS time duration and starts transmitting a data packet to the AP, which will send an ACK frame after a SIFS time interval to confirm that it successfully decoded the data packet.

In order to block the legitimate communications between the source node and the AP of Fig. 23, an intelligent jammer can simply corrupt the RTS/CTS control frames, rather than data packets, which minimizes its energy consumption. There are several different types of intelligent jamming attackers, including the RTS jammer, the CTS jammer, and the ACK jammer. More specifically, 
Table 12 Characteristics of Different Jamming Attacks

\begin{tabular}{|c|c|c|c|c|}
\hline Jammer Types & Energy Efficiency & Jamming Effectiveness & Complexity & Prior Knowledge \\
\hline Constant Jammer & Low & High & Low & Low \\
\hline Intermittent Jammer & Low & Adjustable & Low & Low \\
\hline Reactive Jammer & Moderate & High & Moderate & Moderate \\
\hline Adaptive Jammer & High & High & High & High \\
\hline Intelligent Jammer & High & High & High & High \\
\hline
\end{tabular}

an RTS jammer senses the channel to be idle for a DIFS time period, and then transmits a jamming signal for corrupting a possible RTS packet. By contrast, a CTS jammer attempts to detect the presence of an RTS frame and upon detecting the RTS arrival, it waits for the RTS period plus a SIFS time interval before sending a jamming pulse for disrupting the CTS frame. The CTS jamming strategy will result in a zero throughput for the legitimate transmissions, since no data packets will be transmitted by the source node without successfully receiving a CTS frame. Similarly to the CTS jamming, an ACK jammer also senses the wireless medium. Upon detecting the presence of a packet, it waits for a SIFS time interval at the end of the data packet transmission and then jams the wireless channel, leading to the corruption of an ACK frame. If the source node constantly fails to receive the $\mathrm{ACK}$, it will finally give up transmitting data packets to the AP.

The aforementioned intelligent jammers can be detected by tracing the traffic of MAC control packets to identify abnormal events in terms of sending and/or receiving RTS, CTS, and ACK frames. For example, if the AP (or source node) consistently fails to send and receive the RTS, CTS, or ACK, it may indicate the presence of an intelligent jammer. As mentioned earlier, an intelligent jammer takes advantage of specific upper-layer protocol parameters for significantly degrading the network performance. In order to defend against such an intelligent jammer, a protocol hopping approach, as a generalization of the physical-layer frequency hopping, was proposed in [178], which allows legitimate nodes to hop across various protocol parameters that the jammer may exploit. A game-theoretic framework was formulated in [178] for modeling the interactions between an intelligent jammer and the protocol functions, which was shown to achieve an improved robustness against intelligent jamming attacks.

Finally, Table 12 summarizes the characteristics of the constant, intermittent, reactive, adaptive, and intelligent jammers in terms of their energy efficiency, jamming effectiveness, implementation complexity, and prior knowledge requirements. As shown in Table 12, the constant and intermittent jammers have a low implementation complexity and required no prior knowledge for effectively jamming the legitimate communications, but their energy efficiency is poor. By contrast, the adaptive and intelligent jammers achieve a high energy efficiency and jamming effectiveness, which however, require some prior knowledge (e.g., the legitimate main channel quality, the protocol parameters, etc.) and have a high complexity. As an alternative, the reactive jammer exhibits a high jamming effectiveness and, at the same time, achieves a moderate performance in terms of its energy efficiency, implementation complexity, and prior knowledge requirements.

\section{INTEGRATION OF PHYSICAL-LAYER SECURITY INTO EXISTING WIRELESS NETWORKS}

As discussed earlier, the authentication and encryption constitute a pair of salient techniques adopted in existing wireless security architectures for satisfying the stringent authenticity and integrity requirements of wireless networks (e.g., Wi-Fi, LTE, etc.). Meanwhile, physical-layer security has emerged as a new means of enhancing the security of wireless communications, which is typically considered as a complement to the existing classic authentication and cryptography mechanisms, rather than replacing them [179]. Recently, there have been growing research efforts devoted to the integration of physicallayer security into the existing body of classic wireless authentication and cryptography [179]-[193]. Below we present an in-depth discussion on the physical-layer authentication and cryptography solutions conceived for wireless networks.

Authentication constitutes an essential security requirement designed for reliably differentiating authorized nodes from unauthorized ones in wireless networks. Conventionally, the MAC address of a network node has been used for authentication, which is however, vulnerable to MAC spoofing attacks and can be arbitrarily changed for the sake of impersonating another network node. To this end, an increasing research 
attention has been devoted to the physical-layer authentication [180]-[190] of wireless networks, where either the hardware properties of RF-based devices (also known as device fingerprints) or the propagation characteristics of wireless channels (e.g., the time-varying fading) have been employed for authentication. This line of work is based on the premise that both the device fingerprints and the wireless channels are unique and nonforgeable by an adversary. To elaborate a little further, the random manufacturing imperfections lead to the fact that a pair of RF devices, even produced by the same manufacturing and packaging process, would have different hardware specifications, as exemplified by their clock timing deviations and CFOs, which can be invoked as unique fingerprints for device identification. Additionally, as mentioned in Section V-E, an adversary located at least at a distance of half-a-wavelength away from the legitimate receiver experiences an independent fading channel. This would make it a challenge for the adversary to predict and mimic the wireless channel between the legitimate users, which can thus be used as a unique linkspecific signature for physical-layer authentication.

Specifically, in [180], a clock-timing-based hardware fingerprinting approach was proposed for differentiating the authorized devices from spoofing attackers in Wi-Fi networks, which is passive and noninvasive, hence requiring no extra cooperation from the fingerprintee hosts. It was shown by extensive experiments that the clock-timing-based fingerprint identification is accurate and very effective in differentiating between Wi-Fi devices. Later on, Brik et al. [181] considered the joint use of multiple distinctive radiometric signatures, including the frequency error, synchronization frame correlation, I/Q offset, magnitude error, and phase error, which were inferred from the modulated symbols for identifying different IEEE 802.11 network nodes. This technique was termed as PARADIS [181]. Quantitatively, the experimental results demonstrated that by applying sophisticated machine-learning algorithms, PARADIS was capable of differentiating the legitimate nodes with a probability of at least $99 \%$ in a set of more than 130 network nodes equipped with identical $802.11 \mathrm{NICs}$ in the presence of background noise and wireless fading.

As a design alternative, a CFO-based physical-layer authentication scheme was proposed in [182], where the expected CFO was estimated with the aid of Kalman filtering fed with previous CFO estimates. Then, the expected $\mathrm{CFO}$ was compared to the current $\mathrm{CFO}$ estimate in order to determine, whether the received radio signal obeys a consistent CFO pattern. To be specific, if the difference between the expected $\mathrm{CFO}$ and the current $\mathrm{CFO}$ estimate was higher than a predefined threshold, it indicated the presence of an unknown wireless device. Moreover, the threshold value was adaptively adjusted based on both the background noise level and on the Kalmanprediction-based errors for the sake of further improving the authentication accuracy. Additionally, an SDR-based prototype platform was developed in [182] for validating the feasibility of the proposed CFO-based wireless device authentication in the face of multipath fading channels.

In addition to the device fingerprint-based authentication solutions [180]-[182], the wireless channel is also considered as an effective metric for device authentication [183]-[187]. Specifically, in [183], Xiao et al. studied the employment of channel probing and responses for determining whether an unauthorized user is attempting to invade a wireless network. The reliability of the proposed CSI-based authentication scheme was analyzed in the face of complex Gaussian noise environments. The simulation results relying on the ray-tracing tool WiSE validated the efficiency of the CSI-based authentication approach under a range of realistic practical channel conditions. However, this approach is vulnerable to the so-called mimicry attacker, which is able to forge a CSI signature, as long as the attacker roughly knows the radio signal at the legitimate receiver's location. In order to guard against the mimicry attack, a time-synchronized link signature was presented in [184] by integrating the timing factor into the wireless physical-layer features. The provided experimental results showed that the proposed time-synchronized physical-layer authentication is indeed capable of mitigating the mimicry attack with a high probability.

More recently, in [185], the AoA information was exploited as a highly sensitive physical-layer signature for uniquely identifying each client in IEEE 802.11 networks. To be specific, a multiantenna AP was relied upon, in order to estimate all the directions a client's radio signals arrive from. Once spotting a suspicious transmission, the $\mathrm{AP}$ and the client initiate an AoA signature-based authentication protocol for mitigating the attack. It was shown in [185] that the proposed AoA signature-based authentication scheme was capable of preventing $100 \%$ of Wi-Fi spoofing attacks, while maintaining a false alarm probability of just $0.6 \%$. As a further development, Du et al. [186] examined the extension of physicallayer authentication from single-hop communication networks to dual-hop scenarios by proposing a pair of physical-layer authentication mechanisms, namely the PHY-CRAMR and PHY-AUR techniques for wireless networks operating in the presence of an untrusted relay. The security performance of the PHY-CRAMR and PHY-AUR techniques was analyzed by relying on extensive simulations, showing that both schemes are capable of achieving a high successful authentication probability and a low false alarm rate, especially at sufficiently high SNRs.

It is worth mentioning that all the aforementioned constitutions [180]-[186] have primarily been focused on exploiting the device fingerprints or channel characteristics by relying on their intrinsic randomness. However, these stochastic features are beyond our control. As 
a consequence, in [187]-[189], Yu et al. explored the benefits of a sophisticated deliberate fingerprint embedding mechanism for physical-layer "challenge-response" authentication, which facilitated striking flexible performance tradeoffs by design. More precisely, a stealthy fingerprint was superimposed onto the data in the deliberate fingerprinting mechanism, while additionally both the data and an authentication message were transmitted separately by relying on conventional tag-based authentication methods [190]. Naturally, the authentication message used in conventional tag-based methods reduces the spectral efficiency, while at the same time, being exposed to eavesdropping. By contrast, a deliberately embedded fingerprint can be designed by ensuring that it has high spectral efficiency and remains impervious to eavesdropping. It was shown in [187]-[189] that a compelling tradeoff between the stealth, security, and robustness can be struck by the deliberate fingerprint embedding-based approach in wireless fading environments.

Having presented a range of physical-layer authentication techniques [180]-[190], let us now consider the integration of physical-layer security with classic cryptographic approaches [179], [191]-[193]. Traditionally, the cryptographic techniques relying on secret keys have been employed for protecting the communication confidentiality. However, the distribution and management of secret keys remains quite a challenging task in wireless networks. To this end, Abdallah et al. [179] have investigated the subject of physical-layer cryptography by exploiting the existing ARQ protocol for achieving the reliable exchange of secret keys between the legitimate users without any information leakage to passive eavesdroppers. Specifically, in [191], the secret bits were distributed across the ARQ packets and only the 1-b ACK/NACK feedback from the legitimate receiver was exploited for key sharing. It was shown in [191] that a useful nonzero secrecy rate can be achieved even when the wiretap channel spanning from the source to the eavesdropper has a better condition than the legitimate main channel.

Additionally, Xiao et al. studied the benefits of ARQ mechanisms in terms of generating so-called dynamic secrets by taking advantage of the inevitable information loss in error-prone wireless communications, where the dynamic secrets are constantly extracted from the communication process with the aid of hash functions. ${ }^{1}$ It was shown in [192] that the dynamic secret mechanism is complementary to the family of existing security protocols and it has the benefit of being time-variant, hence remains hard to reveal. However, in [191] and [192], the ARQ feedback was assumed to be perfectly received and decoded without errors, which may not be practical due to the presence of hostile channel impairments. In order

\footnotetext{
${ }^{1} \mathrm{~A}$ hash function is any function that is capable of converting an input data of variable size to an output data of fixed size.
}

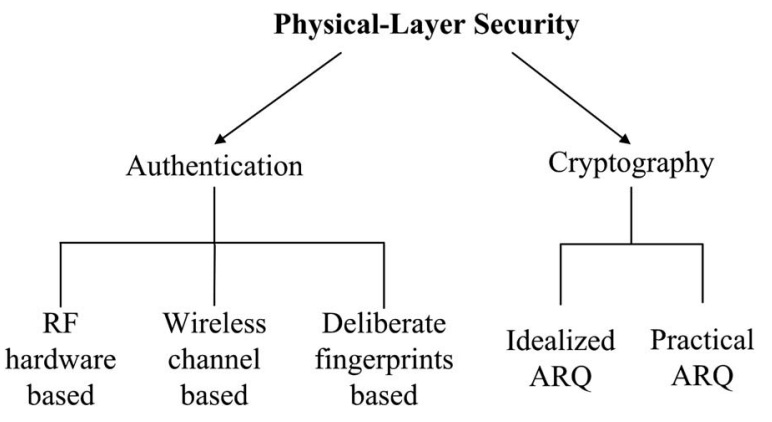

Fig. 24. Classification of physical-layer authentication and cryptography.

to make these investigations more realistic, Khiabani and Wei [193] modeled the practical ARQ feedback channel as a correlated erasure channel and evaluated both the secrecy outage probability and the secrecy capacity of ARQ-aided physical-layer cryptography. It was shown in [193] that a significant secrecy improvement can be achieved even when the eavesdropper's channel conditions are unknown to the legitimate users.

In summary, we have presented an in-depth review concerning the integration of physical-layer security with classic wireless security mechanisms, including physical-layer authentication and cryptography. As shown in Fig. 24, the physical-layer authentication techniques may be classified into three categories, namely the RF-hardwareproperties-based, wireless-channel-characteristics-based, and deliberate-fingerprint-based authentication approaches [180]-[189]. Meanwhile, in the subject of physical-layer cryptography, the existing research efforts [191]-[193] have mainly been focused on exploiting the classic ARQ protocol for securing the exchange of secret keys between legitimate users, where even the realistic practical ARQ feedback associated with transmission errors has been considered.

\section{OPEN CHALLENGES AND FUTURE WORK}

This section presents a range of challenging open issues and future directions for wireless security research. As mentioned in the previous sections, extensive research efforts have been devoted to this subject, but numerous challenges and issues still remain open at the time of writing.

\section{A. Mixed Attacks in Wireless Networks}

Most of the physical-layer security research [119][193] only addressed the eavesdropping attacks, but has neglected the joint consideration of different types of wireless attacks, such as eavesdropping and DoS attacks. It will be of particularly importance to explore new techniques of jointly defending against multiple types of 
wireless attacks, which may be termed as mixed wireless attacks. In order to effectively guard against mixed attacks including both eavesdropping and DoS attacks, we should aim for minimizing the detrimental impact of interference inflicted by DoS attacks on the legitimate transmission. The security defense mechanism should not only consider the CSI of the interfering ink spanning from the DoS attacker to the legitimate receiver, but ideally should also take into account the CSI of the wiretap link between the legitimate transmitter and the eavesdropper, in addition to the CSI of the main link from the legitimate transmitter to the legitimate receiver. It will be of interest to investigate the security defense mechanisms in different scenarios in the presence of both full and partial knowledge of the CSI of the main link as well as of the interfering link and that of the wiretap link. The full CSI-based scenario will provide a theoretical performance upper bound as a guide for developing new signal processing algorithms to guard against mixed attacks. Moreover, considering the fact that the eavesdropper remains silent and the CSI of the wiretap channel is typically unknown, it is of practical interest to conceive security protocols for the scenario, where the eavesdropper's CSI is unavailable.

\section{B. Joint Optimization of Security, Reliability, and Throughput}

Security, reliability, and throughput constitute the main driving factors for the research and development of wireless networks [194]. In conventional wireless systems, the mechanisms assuring security, reliability, and throughput are designed individually and separately, which is however potentially suboptimal, since the three factors are coupled and affect each other [195]. For example, the reliability and throughput of the main link can be improved by increasing the source's transmit power, which however also increases the capacity of the wiretap channel spanning from the source to the eavesdropper and raises the risk that the eavesdropper succeeds in intercepting the source message through the wiretap link. Similarly, although increasing the data rate at $\mathrm{SN}$ improves the security level by reducing the probability of an intercept event, it comes at the expense of a degradation in transmission reliability, since the outage probability of the main link increases for higher data rates. Therefore, it is necessary to investigate the joint optimization of security, reliability, and throughput for the sake of maintaining secure, reliable, and high-rate wireless communications, which is an open challenge to be solved in the future. The goal of the joint optimization is to maximize the wireless security performance under the target reliability and throughput requirements. For example, convex optimization and game theory may be considered for formulating and solving the securityreliability-throughput tradeoff in wireless networks.

\section{Cross-Layer Wireless Security Design and Analysis}

Presently, cross-layer-aided security design is in its infancy. The goal of wireless cross-layer-aided security design is to enable efficient information exchange among different protocol layers for the sake of improving the level of wireless security with minimal network overhead. In general, wireless networks adopt the layered OSI protocol architecture that consists of the physical layer, the MAC layer, the network layer, the transport layer, and the application layer. Traditionally, the aforementioned protocol layers have been protected separately in order to meet their individual communications security requirements, including their authenticity, integrity, and confidentiality [10]. However, these traditional layered security mechanisms are potentially inefficient, since each protocol layer introduces additional computational complexity and latency. For example, in order to meet the authenticity requirements, the existing wireless networks typically adopt multiple authentication approaches at different layers, including MAC-layer authentication, network-layer authentication, and transport-layer authentication. The employment of multiple separate authentication mechanisms at different protocol layers improves the security level of wireless networks, which, however, comes at the expense of a high complexity and latency. As a consequence, it will be of high interest to explore the benefits of cross-layer-aided wireless security in order to guard against the aforementioned mixed wireless attacks. Intuitively, the physical-layer characteristics and properties of wireless channels may also be further exploited by the upper-layer security algorithms, including the user authentication, secret key generation, and data protection algorithms. It is anticipated that the cross-layer security framework will further improve the wireless security at a reduced cost, as compared to the traditional layered security mechanisms.

\section{Physical-Layer Security for the Emerging 5G Systems}

Given the proliferation of smart devices and the increasing demand for multimedia communications, the amount of mobile traffic has substantially grown in recent years and it may soon exceed the capacity of the operational fourth-generation (4G) mobile communications systems [196]. To meet this challenging requirement, substantial efforts have been devoted to the research and development of the fifth-generation (5G) mobile systems [197]-[200] relying on advanced wireless technologies, such as the massive MIMOs and millimeter wave (mmWave) solutions. Meanwhile, it is expected that a strict security requirement is desired for the $5 \mathrm{G}$ systems, since more and more sensitive information (e.g., financial data, personal e-mails, and files) will be transmitted wirelessly [196]-[198]. To this end, physical-layer security as a beneficial complement to conventional security 
mechanisms will have a great potential in the context of $5 \mathrm{G}$ systems. For instance, by deploying a large number of antennas in $5 \mathrm{G}$ systems, the aforementioned artificial noise and beamforming assisted techniques can be readily utilized for improving the transmission performance of legitimate users, while degrading the reception quality of eavesdroppers. However, the application of massive MIMOs for enhancing the physical-layer security also has its own challenges to be addressed, such as the deleterious effects of pilot contamination, power allocation, and channel reciprocity [196]. Therefore, it is of high importance to explore the opportunities and challenges of combining the physical-layer security techniques with 5G enabling technologies, such as massive MIMOs and mmWave solutions.

\section{E. Field Experiments for Physical-Layer Security Investigations}

As discussed above, there are various physical-layer security schemes including the artificial noise, beamforming, and diversity-aided security enhancement approaches, which have been shown to be effective in terms of improving both the secrecy capacity and the secrecy outage probability of wireless communications [126]-[146]. However, their security benefits have so far only been shown theoretically relying on idealized simplifying assumptions, such as the availability of perfect CSI knowledge [201], [202]. By considering the artificial-noise-based method as an example, the accurate CSI of the main channel is required for the appropriate design of an artificial noise, so that the legitimate receiver remains unaffected by the noise, while the eavesdropper is interfered with. However, regardless of the specific channel estimation methods used [148], [149], estimation errors always contaminate the estimation of the CSI, hence the perfect CSI estimation cannot be achieved in practical wireless systems. Given an inaccurate CSI, it is impossible to devise an artificial noise that only interferes with the eavesdropper without affecting the legitimate receiver. Typically, the less accurate the CSI of the main channel, the more interference is received at the legitimate receiver, hence resulting in a degradation of the wireless physical-layer security. Similarly, the CSI estimation errors would also cause a performance degradation for the beamforming- and diversity-aided security approaches. However, it remains unclear to what extent the CSI estimation error affects the attainable physicallayer security performance in terms of the secrecy capacity and secrecy outage probability. It will be of great benefit to conduct field experiments for the sake of verifying the efficiency of various physical-layer security approaches in real wireless communications systems in the presence of both jamming and eavesdropping attacks.

\section{CONCLUSION}

In this paper, we have presented a survey of the wireless security challenges and defense mechanisms conceived for protecting the authenticity, confidentiality, integrity, and availability of wireless transmissions against malicious attacks. We have discussed the range of wireless attacks and security threats potentially experienced at different protocol layers from the application layer to the physical layer, which are classified into application-layer and transport-layer attacks, network-layer, MAC-layer as well as physical-layer attacks. Then, existing security paradigms and protocols conceived for guarding against the different protocol layers' attacks have been reviewed in the context of several widely deployed wireless networks, including the Bluetooth, Wi-Fi, WiMAX, and LTE. Bearing in mind that wireless transmissions are highly vulnerable to eavesdropping attacks owing to the broadcast nature of radio propagation, we have also discussed the state of the art in physical-layer security, which is emerging as a promising paradigm of defending the wireless transmissions against eavesdropping attacks by exploiting the physical-layer characteristics of wireless channels. More specifically, several physical-layer security techniques, including information-theoretic security, artificial-noise-aided security, security-oriented beamforming, diversity-assisted security, and physical-layer secret key generation approaches have been presented as well as compared. Additionally, we have summarized various types of wireless jamming attacks along with their detection and prevention techniques. Finally, we have also discussed the integration of physical-layer security into classic wireless authentication and cryptography, as well as highlighted a range of open challenges to be addressed:

- mixed wireless attacks, where new theories and techniques have to be explored for jointly defending the system against multiple types of wireless attacks;

- joint optimization of security, reliability, and throughput, where an efficient wireless transmission mechanism has to be developed by maximizing the security performance under specific target reliability and throughput requirements;

- cross-layer wireless security design, where a cross-layer security framework has to be investigated for the sake of improving the wireless security at a reduced security overhead and latency as compared to the conventional layered security mechanisms, where the OSI protocol layers are protected separately;

- 5G physical-layer security, where the combination of physical-layer security with $5 \mathrm{G}$ enabling technologies, such as massive MIMOs and mmWave solutions has to be explored for the sake of meeting the strict security requirements imposed by the emerging 5G communication systems; 
- field experiments, where the efficiency of various physical-layer security approaches has to be verified with the aid of field tests in real wireless communications systems without the idealized simplifying assumptions that are routinely used in theoretical studies.

Based on the solutions presented throughout this paper, we provide some general guidelines for wireless communications security design.

- Wireless networks are based on the layered OSI protocol architecture that consists of the application layer, the transport layer, the network layer, the MAC layer, and the physical layer. Each layer will be protected in order to meet the security requirements of wireless networks. Bearing in mind the fact that the different layers support different protocols and exhibit different security vulnerabilities, the security mechanisms invoked by the different wireless protocols should be customized so as to guard against malicious attacks as efficiently as possible.

- The security paradigms, such as user authentication and data encryption used in conventional wireless networks, are typically designed separately at the different protocol layers, which, however, results in high latency and overhead. To this end, cross-layer security would be a candidate for protecting wireless networks against various attacks. To be specific, the physical-layer characteristics of wireless channels may be potentially considered and exploited for designing or customizing the upper-layer security algorithms, including the identity authentication, key generation, and so on.

- The secrecy capacity of wireless communications in the presence of eavesdroppers may be severely degraded due to the time-varying multipath fading effects, which may be significantly mitigated by exploiting a range of diversity-aided techniques, such as time diversity, frequency diversity, and spatial diversity. For example, spatial diversity can be achieved for the sake of attaining the wireless secrecy capacity improvements by using multiple antennas at the legitimate transmitter and/or the legitimate receiver.

- The multiuser scheduling may be employed for the sake of achieving the multiuser diversity gain to improve the wireless secrecy capacity. Additionally, multiuser MIMO may be invoked for further improvements in secrecy capacity, which combines the benefits of the multiuser diversity as well as the MIMO diversity and multiplexing gains.

- Artificial noise generation techniques may be used for improving the wireless physical-layer security against eavesdropping attacks by ensuring that only the eavesdropping attackers are adversely affected by the artificial noise, while the legitimate receiver is unaffected. In order to maximize the security benefits of using the artificial noise assisted method, the power sharing between the desired information-bearing signals and the artificial noise should be given careful attention.

- It is worth mentioning that additional power resources are dissipated in generating the artificial noise to confuse the eavesdropper. Given a fixed total transmit power, increasing the artificial noise power is capable of deteriorating the eavesdropper's channel condition; it, however, comes at the cost of performance degradation of the legitimate receiver, since less transmit power is available for the desired signal transmission. Hence, the power allocation between the artificial noise and desired signal should be carefully considered for the sake of optimizing the wireless physical-layer security.

- Beamforming approaches may also be invoked for improving the wireless security design, which enables the legitimate transmitter to send its information signal in a particular direction to the legitimate receiver by ensuring that the signal received at the legitimate receiver experiences constructive interference, whereas that received at an eavesdropper experiences destructive interference. Moreover, combining the beamforming and the artificial-noise-aided techniques would further enhance the wireless physical-layer security against eavesdropping attacks.

- The security benefits of the artificial noise generation and beamforming techniques are typically maximized at the cost of a throughput or reliability degradation. The conventional mechanisms assuring the security, reliability, and throughput are designed separately, which are not optimized jointly. It is therefore suggested to consider the joint optimization of security, reliability, and throughput for secure wireless communications. For example, the joint optimization problem may be addressed by maximizing the wireless security performance under the target reliability and throughput requirements.

- CSIs of the main channel and/or the wiretap channel are essential in assuring the wireless physical-layer security against eavesdropping attacks. Both the artificial noise and beamformingaided security approaches rely on the CSIs. The accuracy of estimated CSIs has a significant impact on the physical-layer security performance (e.g., the secrecy capacity). It is thus suggested to employ the pilot-based channel estimation approaches, rather than the semiblind or blind channel estimation, for the sake of obtaining accurate CSIs. 
- Performing the accurate channel estimation increases the complexity of the wireless transceiver, especially in fast-fading channels, where the CSI has to be estimated more frequently and the CSI feedback rate has to be increased, resulting in higher transmission overhead in terms of both bandwidth and power. Hence, some balanced system design principles are suggested, where the wireless secrecy capacity may be sacrificed with the intention of reducing the CSI estimation complexity and feedback overhead.

- Physical-layer key generation and agreement techniques are capable of generating secret keys based on the random variations of wireless fading channels for securing wireless networks without the need for a fixed key management infrastructure. However, in static environments, where the wireless nodes are stationary, the channel fading would fluctuate slowly, resulting in a limited number of secret bits to be generated. In these cases, we may consider the employment of MIMO-aided and relay-assisted methods for enhancing the channel's randomness for the sake of improving the secret key generation rate.

- Wireless communications can be disrupted by a jammer at the physical layer by transmitting an interfering signal. Although the FHSS technique is capable of effectively guarding against some of the known physical-layer jamming attacks, the frequency hopping pattern agreement between the legitimate transceivers is challenging in wireless networks. It is therefore advisable to combine FHSS with physical-layer security by exploiting the characteristics of wireless channels for the frequency hopping pattern agreement.

\section{REFERENCES}

[1] O. Aliu, A. Imran, M. Imran, and B. Evans, "A survey of self organisation in future cellular networks," IEEE Commun. Surv. Tut., vol. 15, no. 1, pp. 336-361, Feb. 2013.

[2] H. ElSawy, E. Hossain, and M. Haenggi, "Stochastic geometry for modeling, analysis, and design of multi-tier and cognitive cellular wireless networks: A survey," IEEE Commun. Surv. Tut., vol. 15, no. 3, pp. 996-1019, 3rd Quart. 2013.

[3] ITU, "The World in 2013: ICT facts and figures," Jan. 2013. [Online]. Available: http://www.itu.int/en/ITU-D/Statistics/ Documents/facts/ICTFactsFigures2013.pdf

[4] Symantec Norton Department, "The 2012 Norton cybercrime report," Sep. 2012. [Online]. Available: http://www. norton.com/2012cybercrimereport

[5] M. M. Rashid, E. Hossain, and V. K. Bhargava, "Cross-layer analysis of downlink V-BLAST MIMO transmission exploiting multiuser diversity," IEEE Trans. Wireless Commun., vol. 8, no. 9, pp. 4568-4579, Sep. 2009.

[6] F. Foukalas, V. Gazis, and N. Alonistioti, "Cross-layer design proposals for wireless mobile networks: A survey and taxonomy," IEEE Commun. Surv. Tut., vol. 10, no. 1, pp. 70-85, Apr. 2008.

[7] R. Jurdak, C. Lopes, and P. Baldi, "A survey, classification and comparative analysis of medium access control protocols for ad hoc networks," IEEE Commun. Surv. Tut., vol. 6, no. 1, pp. 2-16, Apr. 2004.

[8] M. Takai, J. Martin, and R. Bagrodia, "Effects of wireless physical layer modeling in mobile ad hoc networks," in Proc. 2nd ACM Int. Symp. Mobile Ad Hoc Netw. Comput., Long Beach, CA, USA, Sep. 2001, pp. 87-94.

[9] C. Saradhi and S. Subramaniam, "Physical layer impairment aware routing (PLIAR) in WDM optical networks: Issues and challenges," IEEE Commun. Surv. Tut., vol. 11, no. 4, pp. 109-130, Dec. 2009.

[10] C. Kolias, G. Kambourakis, and S. Gritzalis, "Attacks and countermeasures on 802.16. Analysis and assessment," IEEE Commun.
Surv. Tut., vol. 15, no. 1, pp. 487-514, Feb. 2013.

[11] M. Stamp, Information Security: Principles and Practice, 2nd ed. New York, NY, USA: Wiley, 2011.

[12] M. Whitman and H. Mattord, Principles of Information Security, 4th ed. Independence, KY, USA: Delmar Cengage Learning, 2012.

[13] Y. Xiao, H.-H. Chen, B. Sun, R. Wang, and S. Sethi, "MAC security and security overhead analysis in the IEEE 802.15.4 wireless sensor networks," EURASIP J.

Wireless Commun. Netw., 2006, doi: 10.1155/WCN/2006/93830.

[14] G. Apostolopoulos, V. Peris, P. Pradhan, and D. Saha, "Securing electronic commerce: Reducing the SSL overhead," IEEE Network, vol. 14, no. 4, pp. 8-16, Jul. 2000.

[15] K. Wong, Y. Zheng, J. Cao, and S. Wang, "A dynamic user authentication scheme for wireless sensor networks," in Proc. IEEE Int. Conf. Sensor Netw. Ubiquitous Trustworthy Comput., Taichung, Taiwan, Jun. 2006, doi: 10.1109/ SUTC.2006.1636182, pp. 244-251.

[16] A. Aziz and W. Diffie, "Privacy and authentication for wireless local area networks," IEEE Pers. Commun., vol. 1, no. 1, pp. 25-31, Aug. 2002.

[17] G. Raju and R. Akbani, "Authentication in wireless networks," in Proc. 40th Annu. Hawai Int. Conf. Syst. Sci., Waikoloa, HI, USA, Jan. 2007, doi: 10.1109/HICSS.2007.93.

[18] L. Venkatraman and D. P. Agrawal, "A novel authentication scheme for ad hoc networks," in Proc. IEEE Wireless Commun Netw. Conf., Chicago, IL, USA, Sep. 2000, pp. 1268-1273.

[19] A. H. Lashkari, K. Lumpur, M. Mansoor, and A. S. Danesh, "Wired equivalent privacy (WEP) versus Wi-Fi protected access (WPA)," in Proc. Int. Conf. Signal Process. Syst., Singapore, May 2009.

[20] K. J. Hole, E. Dyrnes, and P. Thorsheim, "Securing Wi-Fi networks," Computer, vol. 38, no. 7, pp. 28-34, Jul. 2005.

[21] RFC 5246, "The Transport Layer Security (TLS) Protocol Version 1.2", Aug. 2008. [Online]. Available: https://tools.ietf.org/ $\mathrm{html} / \mathrm{rfc5} 246$
[22] RFC 4346, “The Transport Layer Security (TLS) Protocol Version 1.1”, Apr. 2006. [Online]. Available: https://tools.ietf.org/ $\mathrm{html} / \mathrm{rfc} 4346$

[23] RFC 2246, "The Transport Layer Security (TLS) Protocol Version 1.0”, Jan. 1999. [Online]. Available: https://tools.ietf.org/ $\mathrm{html} / \mathrm{rfc} 2246$

[24] S. Lakshmanan, C. Tsao, R. Sivakumar, and K. Sundaresan, "Securing wireless data networks against eavesdropping using smart antennas," in Proc. 28th Int. Conf. Distrib. Comput. Syst., Beijing, China, Jun. 2008, pp. 19-27.

[25] R. Raymond and S. Midkiff, "Denial-ofservice in wireless sensor networks: Attacks and defenses," IEEE Perv. Comput., vol. 7, no. 1, pp. 74-81, Jan. 2008.

[26] B. Kannhavong et al., "A survey of routing attacks in mobile ad hoc networks," IEEE Wireless Commun., vol. 14, no. 5, pp. 85-91, Dec. 2007.

[27] U. Meyer and S. Wetzel, "A man-in-themiddle attack on UMTS," in Proc. 3rd ACM Workshop Wireless Security, Philadelphia, PA, USA, Oct. 2004, pp. 90-97.

[28] T. Ohigashi and M. Morii, "A practical message falsification attack on WPA," in Proc. Joint Workshop Inf. Security, Kaohsiung, Taiwan, Aug. 2009, pp. 1-12.

[29] P. Christof, J. Pelzl, and B. Preneel, Understanding Cryptography: A Textbook for Students and Practitioners. New York, NY, USA: Springer-Verlag, 2010.

[30] C. Elliott, "Quantum cryptography," IEEE Security Privacy, vol. 2, no. 4, pp. 57-61, Apr. 2004.

[31] Q. Wang, K. Xu, and K. Ren, "Cooperative secret key generation from phase estimation in narrowband fading channels," IEEE J. Sel. Areas Commun., vol. 30, no. 9, pp. 1666-1674, Sep. 2012.

[32] Y. Wei, K. Zengy, and P. Mohapatra, "Adaptive wireless channel probing for shared key generation," in Proc. 30th Annu. IEEE Int. Conf. Comput. Commun., Shanghai, China, Apr. 2011, pp. 2165-2173.

[33] A. D. Wyner, "The wire-tap channel," Bell Syst. Tech. J., vol. 54, no. 8, pp. 1355-1387, 1975. 
[34] S. K. Leung-Yan-Cheong and M. E. Hellman, "The Gaussian wiretap channel," IEEE Trans. Inf. Theory, vol. 24, no. 7, pp. 451-456, Jul. 1978.

[35] Y. Zou, X. Wang, and W. Shen, "Intercept probability analysis of cooperative wireless networks with best relay selection in the presence of eavesdropping attack," in Proc. IEEE Int. Conf. Commun., Budapest, Hungary, Jun. 2013, pp. 2183-2187.

[36] Y. Zou, X. Wang, and W. Shen, "Eavesdropping attack in collaborative wireless networks: Security protocols and intercept behavior," in Proc. 17th IEEE Int. Conf. Comput. Supported Cooperative Work in Design, Whistler, Canada, Jun. 2013, pp. 704-709.

[37] O. Cepheli, T. Maslak, and G. Kurt, "Analysis on the effects of artificial noise on physical layer security," in Proc. 21st Signal Process. Commun. Appl. Conf., Haspolat, Turkey, Apr. 2013, pp. 1-4.

[38] A. Araujo, J. Blesa, E. Romero, and O. Nieto-Taladriz, "Artificial noise scheme to ensure secure communications in CWSN," in Proc. 8th Int. Wireless Commun. Mobile Comput. Conf., Limassol, Cyprus, Aug. 2012, pp. 1023-1027.

[39] N. Romero-Zurita, M. Ghogho, and D. McLernon, "Outage probability based power distribution between data and artificial noise for physical layer security," IEEE Signal Process. Lett., vol. 19, no. 2, pp. 71-74, Feb. 2012.

[40] J. Wu and J. Chen, "Multiuser transmit security beamforming in wireless multiple access channels," in Proc. IEEE Int. Conf. Commun., Ottawa, Canada, ON, Jun. 2012, pp. 903-906.

[41] H. Wang, Q. Yin, and X. Xia, "Distributed beamforming for physical-layer security of two-way relay networks," IEEE Trans. Signal Process., vol. 60, no. 7 , pp. 3532-3545, Jul. 2012.

[42] Y. Zou, X. Wang, and W. Shen, "Optimal relay selection for physical-layer security in cooperative wireless networks," IEEE J. Sel. Areas Commun., vol. 31, no. 10, pp. 2099-2111, Oct. 2013.

[43] Y. Zou, X. Wang, and W. Shen, "Physical-layer security with multiuser scheduling in cognitive radio networks," IEEE Trans. Commun., vol. 61 , no. 12, pp. 5103-5113, Dec. 2013.

[44] D. Ma and G. Tsudik, "Security and privacy in emerging wireless networks," IEEE Wireless Commun., vol. 17, no. 5, pp. 12-21, Oct. 2010.

[45] H. Kumar, D. Sarma, and A. Kar, "Security threats in wireless sensor networks," IEEE Aerosp. Electron. Syst. Mag., vol. 23, no. 6, pp. 39-45, Jun. 2008.

[46] Y. Shiu et al., "Physical layer security in wireless networks: A tutorial," IEEE Wireless Commun., vol. 18, no. 2, pp. 66-74, Apr. 2011.

[47] Y. Jiang, C. Lin, X. Shen, and M. Shi, "Mutual authentication and key exchange protocols for roaming services in wireless mobile networks," IEEE Trans. Wireless Commun., vol. 5, no. 9, pp. 2569-2577, Sep. 2006.

[48] W. Stalling, Cryptography and Network Security: Principles and Practices, 3rd ed. Englewood Cliffs, NJ, USA: Prentice-Hall, Jan. 2010.

[49] X. Chen, K. Makki, K. Yen, and N. Pissinou, "Sensor network security: A survey," IEEE Commun. Surv. Tut., vol. 11, no. 2, pp. 52-73, Apr. 2009.
[50] D. Dzung, M. Naedele, T. Von Hoff, and M. Crevatin, "Security for industrial communications systems," Proc. IEEE, vol. 93, no. 6, pp. 1152-1177, Jun. 2005.

[51] E. Shi and A. Perrig, "Designing secure sensor networks," IEEE Wireless Commun., vol. 11, no. 6, pp. 38-43, Dec. 2004.

[52] X. Lin, "CAT: Building couples to early detect node compromise attack in wireless sensor networks," in Proc. IEEE Global Telecommun. Conf., Honolulu, HI, USA, Dec. 2009, pp. 1-6.

[53] A. D. Wood and J. A. Stankovic, "Denial of service in sensor networks," IEEE Computer, vol. 35, no. 10, pp. 54-62, Oct. 2002.

[54] H. Huang, N. Ahmed, and P. Karthik, "On a new type of denial of service attack in wireless networks: The distributed jammer network," IEEE Trans. Wireless Commun., vol. 10, no. 7, pp. 2316-2324, Jul. 2011.

[55] J. Ren and T. Li, "CDMA physical layer built-in security enhancement," in Proc. IEEE 58th Veh. Technol. Conf., Orlando, FL, USA, Oct. 2003, pp. 2157-2161.

[56] A. Mpitziopoulos, G. Pantziou, and C. Konstantopoulos, "Defending wireless sensor networks from jamming attacks," in Proc. IEEE 18th Int. Symp. Pers. Indoor Mobile Radio Commun., Athens, Greece, Jan. 2013, pp. 1-5.

[57] K. Pelechrinis, C. Koufogiannakis, and S. Krishnamurthy, "On the efficacy of frequency hopping in coping with jamming attacks in 802.11 networks," IEEE Trans. Wireless Commun., vol. 9, no. 10, pp. 3258-3271, Oct. 2010.

[58] IEEE Standard for Telecommunications and Information Exchange Between Systems-LAN/MAN-Specific Requirements-Part 15.1: Wireless Medium Access Control (MAC) and Physical Layer (PHY) Specifications for Wireless Personal Area Networks (WPANs), IEEE 802.15.1 Working Group.2005.

[59] IEEE Standard for Information Technology-Telecommunications and Information Exchange Between Systems-Local and Metropolitan Area Networks-Specific Requirements-Part 11: Wireless LAN Medium Access Control (MAC) and Physical Layer (PHY) Specifications, IEEE 802.11 Working Group.2007.

[60] IEEE Standard for Local and Metropolitan Area Networks Part 16: Air Interface for Broadband Wireless Access Systems Amendment 3: Advanced Air Interface, IEEE 802.16m Working Group.2011.

[61] A. Ghosh et al., "LTE-advanced: Next-generation wireless broadband technology," IEEE Wireless Commun., vol. 17, no. 3, pp. 10-22, Jun. 2010.

[62] B. Haibo, L. Sohraby, and C. Wang, "Future internet services and applications," IEEE Network, vol. 24, no. 4, pp. 4-5, Apr. 2010.

[63] R. Bruno and M. Conti, "Throughput analysis and measurements in IEEE 802.11 WLANs with TCP and UDP traffic flows," IEEE Trans. Mobile Comput., vol. 7, no. 2, pp. 171-186, Feb. 2008.

[64] S. Lee, G. Ahn, and A. Campbell, "Improving UDP and TCP performance in mobile ad hoc networks with INSIGNIA," IEEE Commun. Mag., vol. 39, no. 6, pp. 156-165, Jun. 2001.

[65] C. Labovitz, A. Ahuja, and F. Jahanian, "Delayed Internet routing convergence," IEEE/ACM Trans. Netw., vol. 9, no. 3, pp. 293-306, Aug. 2008.
[66] R. Derryberry et al., "Transmit diversity in 3G CDMA systems," IEEE Commun. Mag., vol. 40, no. 4, pp. 68-75, Apr. 2002.

[67] D. Bai et al., "LTE-advanced modem design: Challenges and perspectives," IEEE Commun. Mag., vol. 50, no. 2, pp. 178-186, Feb. 2012.

[68] S. M. Bellovin, "Security problems in the TCP/IP protocol suite," ACM SIGCOMM Comput. Commun. Rev., vol. 19, no. 2, pp. 32-48, Apr. 1989.

[69] G. Zargar and P. Kabiri, "Identification of effective network features to detect Smurf attacks," in Proc. IEEE Student Conf. on Res. Develop., Serdang, Malaysia, Nov. 2009, pp. $49-52$.

[70] T. Shon and W. Choi, "An analysis of mobile WiMAX security: Vulnerabilities and solutions," in Network-Based Information Systems Lecture Notes in Computer Science. Berlin, Germany: Springer-Verlag, 2007, vol. 4658, pp. 88-97.

[71] A. Perrig, J. Stankovic, and D. Wagner, "Security in wireless sensor networks," Commun. ACM, vol. 47 , no. 6 , pp. 53-57, Jun. 2004.

[72] A. Mpitziopoulos, "A survey on jamming attacks and countermeasures in WSNs," IEEE Commun. Surv. Tut., vol. 11, no. 4, pp. 42-56, Dec. 2009.

[73] V. Nagarajan and D. Huang, "Using power hopping to counter MAC spoof attacks in WLAN," in Proc. IEEE Consumer Commun. Netw. Conf., Las Vegas, NV, USA, Jan. 2010, pp. 1-5.

[74] W. Zhou, A. Marshall, and Q. Gu, "A novel classification scheme for 802.11 WLAN active attacking traffic patterns," in Proc. IEEE Wireless Commun. Netw. Conf., Las Vegas, NV, USA, Apr. 2006, pp. 623-628.

[75] J. Park and S. Kasera, "Securing Ad Hoc wireless networks against data injection attacks using firewalls," in Proc. IEEE Wireless Commun. Netw. Conf., Hongkong, China, Apr. 2007, pp. 2843-2848.

[76] Computer Emergency Response Team (CERT), "CERT Advisory: IP Spoofing Attacks and Hijacked Terminal Connections," Jan. 1995. [Online]. Available: http://www.cert.org/advisories/ CA-1995-01.html

[77] N. Hastings and P. McLean, "TCP/IP spoofing fundamentals," in Proc. IEEE 15th Annu. Int. Conf. Comput. Commun., Phoenix, AZ, USA, Mar. 1996, pp. 218-224.

[78] B. Harrisa and R. Hunt, "TCP/IP security threats and attack methods," Comput. Commun., vol. 22, no. 10, pp. 885-897, Jun. 1999.

[79] F. El-Moussa, N. Linge, and M. Hope, "Active router approach to defeating," denial-of-service attacks in networks," IET Commun., vol. 1, no. 1, pp. 55-63, Feb. 2007.

[80] C. Schuba et al., "Analysis of a denial of service attack on TCP," in Proc. IEEE Symp. Security Privacy, Oakland, USA, May 1997, pp. 208-223.

[81] A. Kuzmanovic and E. W. Knightly, "Low-rate TCP-targeted denial of service attacks and counter strategies," IEEE/ACM Trans. Netw., vol. 14, no. 4, pp. 683-696, Aug. 2006.

[82] R. Chang, "Defending against flooding-based distributed denial-of-service attacks: A tutorial," IEEE Commun. Mag., vol. 40 no. 10 , pp. $42-51$, Oct. 2002. 
[83] RFC 2577, "FTP security considerations," May 1999. [Online]. Available: http://tools. ietf.org/html/rfc2577

[84] T. Bass, A. Freyre, D. Gruber, and G. Watt, "E-mail bombs and countermeasures: Cyber attacks on availability and brand integrity," IEEE Network, vol. 12, no. 2, pp. 10-17, Mar. 1998.

[85] A. Kieyzun, P. Guo, K. Jayaraman, and M. Ernst, "Automatic creation of SQL injection and cross-site scripting attacks," in Proc. IEEE 31st Int. Conf. Softw. Eng., Vancouver, Canada BC, May 2009, pp. 199-209.

[86] C. Stevenson et al., "IEEE 802.22: The first cognitive radio wireless regional area network standard," IEEE Commun. Mag., vol. 47, no. 1, pp. 130-138, Jan. 2009.

[87] E. Ferro and F. Potorti, "Bluetooth and Wi-Fi wireless protocols: A survey and a comparison," IEEE Wireless Commun. vol. 12, no. 1, pp. 12-26, Feb. 2005.

[88] M. Polla, F. Martinelli, and D. Sgandurra, "A Survey on security for mobile devices," IEEE Commun. Surv. Tut., vol. 15, no. 1 , pp. 446-471, Mar. 2013.

[89] D. Pareit, I. Moerman, and P. Demeester, "The history of WiMAX: A complete survey of the evolution in certification and standardization for IEEE 802.16 and WiMAX," IEEE Commun. Surv. Tut., vol. 14, no. 4, pp. 1183-1211, Oct. 2012.

[90] J. Cao, H. Ma, H. Li, and Y. Zhang, "A survey on security aspects for LTE and LTE-A networks," IEEE Commun. Surv. Tut., vol. 15, no. 2, Apr. 2013.

[91] T. Muller, "Bluetooth Security Architecture," Jul. 1999. [Online]. Available: http://www.afn.org/afn48922/ downs/wireless/1c11600.pdf

[92] M. Kui and X. Cuo, "Research of Bluetooth security manager," in Proc. IEEE Int. Conf. Neural Netw. Signal Process., Nanjing, China, Dec. 2003, pp. 1681-1684.

[93] P. Toengel, "Bluetooth: Authentication, athorisation and encryption," Jul. 1999. [Online]. Available: http://www.toengel. net/studium/mm_and_sec/bluetooth.pdf

[94] A. Lashkari, M. Danesh, and B. Samadi, "A survey on wireless security protocols (WEP, WPA and WPA2/802.11i)," in Proc. 2nd IEEE Int. Conf. Comput. Sci. Inf. Technol., Beijing, China, Aug. 2009, pp. 48-52.

[95] A. Lashkari, M. Mansoor, and A. Danesh, "Wired equivalent privacy (WEP) versus Wi-Fi protected access (WPA)," in Proc. Int. Conf. Signal Process. Syst., Singapore, May 2009, pp. 445-449.

[96] J. Lee and C. Fan, "Efficient low-latency RC4 architecture designs for IEEE 802.11i WEP/TKIP," in Proc. Int. Symp. Intell. Signal Process. Commun. Syst., Xiamen, China, Nov. 2007, pp. 56-59.

[97] A. Stubbleleld, J. Ioannidis, and A. D. Rubin, "A key recovery attack on the $802.11 \mathrm{~b}$ wired equivalent privacy protocol (WEP)," ACM Trans. Inf. Syst. Security, vol. 7, no. 2, pp. 319-332, May 2004.

[98] E. Tews, R.-P. Weinmann, and A. Pyshkin, "Breaking 104 bit WEP in less than 60 seconds," in Information Security Applications. Lecture Notes in Computer Science, Berlin, Germany: Springer-Verlag, 2007, vol. 4867, pp. 188-202.

[99] J. Lin, Y. Kao, and C. Yang, "Secure enhanced wireless transfer protocol," in Proc. 1st Int. Conf. Availab. Reliab. Security, Vienna, Austria, Apr. 2006, pp. 536-543.
[100] C. Nancy, H. Russ, W. David, and W. Jesse, "Security flaws in 802.11 data link protocols," Commun. ACM, vol. 46, no. 5, pp. 35-39, May 2003.

[101] E. Tews and M. Beck, "Practical attacks against WEP and WPA," in Proc. 2nd ACM Conf. Wireless Netw. Security, Zurich, Switzerland, Mar. 2009, pp. 79-86.

[102] Q. Li, X. Lin, J. Zhang, and W. Roh, "Advancement of MIMO technology in WiMAX: From IEEE $802.16 \mathrm{~d} / \mathrm{e} / \mathrm{j}$ to 802.16m," IEEE Commun. Mag., vol. 47, no. 6, pp. 100-107, Jun. 2009.

[103] T. Han et al., "Analysis of mobile WiMAX security: Vulnerabilities and solutions," in Proc. 5th IEEE Int. Conf. Mobile Ad Hoc Sensor Syst., Atlanta, GA, USA, Sep. 2008, pp. 828-833.

[104] F. Yang, "Comparative analysis on TEK exchange between PKMv1 and PKMV2 for WiMAX," in Proc. 7th Int. Conf. Wireless Commun. Netw. Mobile Comput., Wuhan, China, Sep. 2011, pp. 1-4.

[105] D. Johnston and J. Walker, "Overview of IEEE 802.16 security," IEEE Security Privacy, vol. 2, no. 3, pp. 40-48, Jun. 2004.

[106] S. Adibi et al., "Authentication authorization and accounting (AAA) schemes in WiMAX," in Proc. IEEE Int. Conf. Electroinf. Technol., May 2006, pp. 210-215.

[107] E. Biham, "A fast new DES implementation in software," in Proc. 4th Int. Workshop Fast Softw. Encryption, Haifa, Israel, Jan. 1997, pp. 260-272.

[108] S. Ahson and M. Ilyas, WiMAX Standards and Security, Boca Raton, FL: CRC Press, 2007.

[109] D. Astely et al., "LTE: The evolution of mobile broadband," IEEE Commun. Mag., vol. 47, no. 4, pp. 44-51, Apr. 2009.

[110] The 3rd Generation Partnership Project (3GPP), "Technical specification group services and system aspects: Service requirements for Home Node B (HNB) and home eNode B (HeNB) (Rel 11)," Sep. 2012.

[111] M. Zhang and Y. Fang, "Security analysis and enhancements of 3GPP authentication and key agreement protocol," IEEE Trans. Wireless Commun., vol. 4, no. 2 , pp. 734-742, Mar. 2005.

[112] G. M. Koien, "Mutual entity authentication for LTE," in Proc. 7th Int. Wireless Commun. Mobile Comput. Conf., Istanbul, Turkey, Jul. 2011, pp. 43-55.

[113] The 3rd Generation Partnership Project (3GPP), "Technical specification group services and system aspects: 3G security, specification of the 3GPP confidentiality and integrity algorithms," Document 2: KASUMI Specification, 2001.

[114] A. Kircanski and A. Youssef, "On the sliding property of SNOW $3 \mathrm{G}$ and SNOW 2.0," IET Inf. Security, vol. 5, no. 4, pp. 199-206, Dec. 2011.

[115] B. Sklar, "Rayleigh fading channels in mobile digital communication systemsPart I: Characterization," IEEE Commun. Mag., vol. 35, no. 7, pp. 90-100, Jul. 1997.

[116] A. Abdi, C. Tepedelenlioglu, M. Kaveh, and G. Giannakis, "On the estimation of the $\mathrm{K}$ parameter for the rice fading distribution," IEEE Commun. Lett., vol. 5, no. 3, pp. 92-94, Mar. 2001.

[117] M. D. Yacoub, J. E. V. Bautista, and L. Guerra de Rezende Guedes, "On higher order statistics of the Nakagami-m distribution," IEEE Trans. Veh. Technol., vol. 48, no. 3, pp. 790-794, May 1999.
[118] S. Goel and R. Negi, "Secret communication in presence of colluding eavesdroppers," in Proc. IEEE Military Commun. Conf., Atlantic City, NJ, USA, 2005, pp. 1501-1506.

[119] C. E. Shannon, "Communications theory of secrecy systems," Bell Syst. Tech. J., vol. 28, pp. 656-715, 1949.

[120] A. Khisti, G. Womell, A. Wiesel, and Y. Eldar, "On the Gaussian MIMO wiretap channel," in Proc. IEEE Int. Symp. Inf. Theory, Nice, France, Jun. 2007, pp. 2471-2475.

[121] A. Khisti and G. W. Wornell, "Secure transmission with multiple antennas: The MISOME wiretap channel," IEEE Trans. Inf. Theory, vol. 56, no. 7, pp. 3088-3104, Jul. 2010.

[122] A. Khisti and G. W. Wornell, "Secure transmission with multiple antennas-Part II: The MIMOME wiretap channel," IEEE Trans. Inf. Theory, vol. 56, no. 11, pp. 5515-5532, Nov. 2010.

[123] T. Chrysikos, T. Dagiuklas, and S. Kotsopoulos, "A closed-form expression for outage secrecy capacity in wireless information-theoretic security," in Proc. 1st Int. ICST Workshop Security Emerging Wireless Commun. Netw. Syst., Athens, Greece, Sep. 2009, pp. 3-12.

[124] F. Oggier and B. Hassibi, "The secrecy capacity of the MIMO wiretap channel," IEEE Trans. Inf. Theory, vol. 57, no. 8, pp. 4961-4972, Aug. 2011.

[125] X. He, A. Khisti, and A. Yener, "MIMO broadcast channel with arbitrarily varying eavesdropper channel: Secrecy degrees of freedom," in Proc. IEEE Global Telecommun. Conf., Houston, TX, USA, Dec. 2011, pp. 1-5.

[126] S. Goel and R. Negi, "Guaranteeing secrecy using artificial noise," IEEE Trans. Wireless Commun., vol. 7, no. 6, pp. 2180-2189, Jul. 2008.

[127] X. Zhou and M. McKay, "Secure transmission with artificial noise over fading channels: Achievable rate and optimal power allocation," IEEE Trans. Veh. Technol., vol. 59, no. 8, pp. 3831-3842, Aug. 2010

[128] W. Liao, T. Chang, W. Ma, and C. Chi "QoS-based transmit beamforming in the presence of eavesdroppers: An optimized artificial-noise-aided approach," IEEE Trans. Veh. Technol., vol. 59, no. 3, pp. 1202-1216, Mar. 2011.

[129] Q. Li and W. Ma, "A robust artificial noise aided transmit design for MISO secrecy," in Proc. IEEE Int. Conf. Acoust. Speech Signal Process., Prague, Czech Republic, May 2011, pp. 3436-3439.

[130] D. Goeckel et al., "Artificial noise generation from cooperative relays for everlasting secrecy in two-hop wireless networks," IEEE J. Sel. Areas Commun., vol. 29, no. 10, pp. 2067-2076, Oct. 2011.

[131] J. Zhang and M. Gursoy, "Collaborative relay beamforming for secrecy," in Proc. IEEE Int. Conf. Commun., Cape Town, South Africa, May 2010, pp. 1-5.

[132] A. Mukherjee and A. Swindlehurst, "Robust beamforming for security in MIMO wiretap channels with imperfect CSI," IEEE Trans. Signal Process., vol. 59, no. 1, pp. 351-361, Jan. 2011.

[133] C. Jeong, I. Kim, and K. Dong, "Joint secure beamforming design at the source and the relay for an amplify-and-forward MIMO untrusted relay system," IEEE Trans. Signal Process., vol. 60, no. 1 , pp. 310-325, Jan. 2012. 
[134] N. Anand, S.-J. Lee, and E. W. Knightly, "Strobe: Actively securing wireless communications using zero-forcing beamforming," in Proc. 31st IEEE INFOCOM, Orlando, FL, USA, Mar. 2012, pp. 720-728.

[135] H. Qin et al., "Optimal power allocation for joint beamforming and artificial noise design in secure wireless communications," in Proc. IEEE Int. Conf. Commun. Workshops, Kyoto, Japan, Jun. 2011, pp. 1-5.

[136] N. Romero-Zurita, M. Ghogho, and D. McLernon, "Outage probability based power distribution between data and artificial noise for physical layer security," IEEE Signal Process. Lett., vol. 19, no. 2, pp. 71-74, Feb. 2012.

[137] Y. Zou, J. Zhu, X. Wang, and V. Leung, "Improving diversity for physical-layer security in wireless communications," IEEE Network, vol. 29 , no. 1 , pp. $42-48$, Jan. 2015.

[138] L. Zheng and D. Tse, "Diversity and multiplexing: A fundamental tradeoff in multiple-antenna channels," IEEE Trans. Inf. Theory, vol. 49, no. 5, pp. 1073-1096, May 2003.

[139] S. Alamouti, "A simple transmitter diversity scheme for wireless communications," IEEE J. Sel. Areas Commun., vol. 16, no. 8 , pp. 1451-1458, Oct. 1998.

[140] S. Srikanth, P. A. Murugesa, and $\mathrm{X}$. Fernando, "Orthogonal frequency division multiple access in WiMAX and LTE: A comparison," IEEE Commun. Mag., vol. 50, no. 9, pp. 153-161, Sep. 2012.

[141] J. Laiho, K. Raivio, P. Lehtimaki, K. Hatonen, and O. Simula, "Advanced analysis methods for 3G cellular networks," IEEE Trans. Wireless Commun., vol. 4, no. 3, pp. 930-942, Jun. 2005.

[142] Y. Zou, X. Wang, and W. Shen, "Physical-layer security with multiuser scheduling in cognitive radio networks," IEEE Trans. Commun., vol. 61, no. 12, pp. 5103-5113, Dec. 2013.

[143] A. Sendonaris, E. Erkip, and B. Aazhang, "User cooperation diversity-Part I: System description," IEEE Trans. Commun., vol. 51, no. 11, pp. 1927-1938, Nov. 2003.

[144] Y. Zou, Y.-D. Yao, and B. Zheng, "Opportunistic distributed space-time coding for decode-and-forward cooperation systems," IEEE Trans. Signal Process., vol. 60, no. 4, pp. 1766-1781, Apr. 2012.

[145] Y. Zou, X. Li, and Y.-C. Liang, "Secrecy outage and diversity analysis of cognitive radio systems," IEEE J. Sel. Areas Commun., vol. 32, no. 11, pp. 2222-2236, Nov. 2014.

[146] K. Ren, H. Su, and Q. Wang, "Secret key generation exploiting channel characteristics in wireless communications," IEEE Wireless Commun., vol. 18, no. 4, pp. 6-12, Aug. 2011.

[147] Y. Li, L. J. Cimini, and N. R. Sollenberger, "Robust channel estimation for OFDM systems with rapid dispersive fading channels," IEEE Trans. Commun., vol. 46, no. 7, pp. 902-915, Aug. 2002.

[148] B. Muquet, M. Courville, and P. Duhamel, "Subspace-based blind and semi-blind channel estimation for OFDM systems," IEEE Trans. Signal Process., vol. 50, no. 7, pp. 1699-1712, Jul. 2002

[149] M. Steiner, G. Tsudik, and M. Waidner, "Diffie-Hellman key distribution extended to group communication," in Proc. 3rd ACM Conf. Comput. Commun. Security, New Delhi, India, Mar. 1996, pp. 31-37.
[150] J. E. Hershey, A. A. Hassan, and R. Yarlagadda, "Unconventional cryptographic keying variable management," IEEE Trans. Commun., vol. 43, no. 1, pp. 3-6, Jan. 1995.

[151] A. A. Hassan, W. E. Stark, J. E. Hershey, and S. Chennakeshu, "Cryptographic key agreement for mobile radio," Digital Signal Process., vol. 6, no. 10, pp. 207-212, Oct. 1996.

[152] S. Mathur, W. Trappe, N. Mandayam, C. Ye, and A. Reznik, "Radio-telepathy: Extracting a secret key from an unauthenticated wireless channel," in Proc. 14th Annu. Int. Conf. Mobile Comput. Netw., San Francisco, CA, USA, Sep. 2008, pp. 128-139.

[153] S. Jana et al., "On the effectiveness of secret key extraction from wireless signal strength in real environments," in Proc. 15th Annu. Int. Conf. Mobile Comput. Netw., Beijing, China, Sep. 2009, pp. 321-332.

[154] S. Gollakota and D. Katabi, "Physical layer wireless security made fast and channel independent," in Proc. 30th Annu. IEEE Int. Conf. Comput. Commun., Shanghai, China, Apr. 2011, pp. 1125-1133.

[155] H. Liu, J. Yang, Y. Wang, and Y. Chen, "Collaborative secret key extraction leveraging received signal strength in mobile wireless networks," in Proc. 31st Annu. IEEE Int. Conf. Comput. Commun., Orlando, FL, USA, Mar. 2012, pp. 927-935.

[156] Y. Shehadeh, O. Alfandi, K. Tout, and D. Hogrefe, "Intelligent mechanisms for key generation from multipath wireless channels," in Proc. Wireless Telecommun. Symp., New York, NY, USA, Apr. 2011, pp. 1-6.

[157] Q. Wang, H. Su, K. Ren, and K. Kim, "Fast and scalable secret key generation exploiting channel phase randomness in wireless networks," in Proc. 30th Annu. IEEE Int. Conf. Comput. Commun., Shanghai, China, Mar. 2011, pp. 1422-1430.

[158] J. W. Wallace, C. Chen, and M. A. Jensen, "Key generation exploiting MIMO channel evolution: Algorithm and theoretical limits," in Proc. 3rd Eur. Conf. Antennas Propag., Berlin, Germany, Mar. 2009, pp. 1499-1503.

[159] K. Zeng, D. Wu, A. Chan, and P. Mohapatra, "Exploiting multiple-antenna diversity for shared secret key generation in wireless networks," in Proc. 29th Annu. IEEE Int. Conf. Comput. Commun., San Diego, CA, USA, Mar. 2010.

[160] T. Shimizu, H. Iwai, and H. Sasaoka, "Physical-layer secret key agreement in two-way wireless relaying systems," IEEE Trans. Inf. Forens. Security, vol. 6, no. 3 , pp. 650-660, Sep. 2011.

[161] M. Edman, A. Kiayias, and B. Yener, "On passive inference attacks against physical-layer key extraction?" in Proc. 4th Eur. Workshop Syst. Security, Salzburg, Austria, Apr. 2011, doi: 10.1145/1972551.1972559.

[162] S. Eberz, M. Strohmeier, M. Wilhelm, and I. Martinovic, "A practical man-in-the-middle attack on signal-based key generation protocols," Comput. Security, pp. 235-252, 2012.

[163] L. Shi et al., "ASK-BAN: Authenticated secret key extraction utilizing channel characteristics for body area networks," in Proc. 6th ACM Conf. Security Privacy Wireless Mobile Netw., Budapest, Hungary, Apr. 2013, pp. 155-166.
[164] K. Pelechrinis, M. Iliofotou, and S. V. Krishnamurthy, "Denial of service attacks in wireless networks: The case of jammers," IEEE Commun. Surv. Tut., vol. 13, no. 2, pp. 245-257, May 2011.

[165] T. X. Brown, J. E. James, and A. Sethi, "Jamming and sensing of encrypted wireless ad hoc networks," in Proc. 7th ACM Int. Symp. Mobile Ad Hoc Netw. Comput., Florence Italy, May 2006, pp. 120-130.

[166] W. Xu, W. Trappe, Y. Zhang, and T. Wood, "The feasibility of launching and detecting jamming attacks in wireless networks," in Proc. 6th ACM Int. Symp. Mobile Ad Hoc Netw. Comput., Urbana-Champaign, IL, USA, May 2005, pp. 46-57.

[167] D. J. Torrieri, "Frequency hopping with multiple frequency-shift keying and hard decisions," IEEE Trans. Commun., vol. 32, no. 5, pp. 574-582, May 1984.

[168] V. Navda, A. Bohra, S. Ganguly, and D. Rubenstein, "Using channel hopping to increase 802.11 resilience to jamming attacks," in Proc. 26th Annu. IEEE Int. Conf. Comput. Commun., Anchorage, AK, USA, May 2007, pp. 2526-2530.

[169] R. Gummadi, D. Wetheral, B. Greenstein, and S. Seshan, "Understanding and mitigating the impact of RF interference on 802.11 networks," in Proc. ACM SIGCOMM 2007, Kyoto, Japan, Aug. 2007, pp. 385-396.

[170] O. Besson, P. Stoica, and Y. Kamiya, "Direction finding in the presence of an intermittent interference," IEEE Trans. Signal Process., vol. 50, no. 7 , pp. 1554-1564, Jul. 2002.

[171] Y. Liu and P. Ning, "BitTrickle: Defending against broadband and high-power reactive jamming attacks," in Proc. 31st Annu. IEEE Int. Conf. Comput. Commun., Orlando, FL, USA, Mar. 2012, pp. 909-917.

[172] E. Lance and G. K. Kaleh, "A diversity scheme for a phase-coherent frequency-hopping spread-spectrum system," IEEE Trans. Commun., vol. 45, no. 9, pp. 1123-1129, Sep. 1997.

[173] L. Freitag, M. Stojanovic, S. Singh, and M. Johnson, "Analysis of channel effects on direct-sequence and frequency-hopped spread-spectrum acoustic communication," IEEE J. Ocean. Eng., vol. 26, no. 4, pp. 586-593, Oct. 2001.

[174] J. Jeung, S. Jeong, and J. Lim, “Adaptive rapid channel-hopping scheme mitigating smart jammer attacks in secure WLAN," in Proc. Military Commun. Conf., Baltimore, MD, USA, Nov. 2011, pp. 1231-1236.

[175] Z. Liu, H. Liu, W. Xu, and Y. Chen, "An error-minimizing framework for localizing jammers in wireless networks," IEEE Trans. Parallel Distrib. Syst., vol. 25, no. 2, pp. 508-517, Feb. 2014.

[176] B. P. Crow, I. Widjaja, J. G. Kim, and P. T. Sakai, "IEEE 802.11 wireless local area networks," IEEE Commun. Mag., vol. 35, no. 9, pp. 116-126, Sep. 1997.

[177] G. Bianchi, "Performance analysis of the IEEE 802.11 distributed coordination function," IEEE J. Sel. Areas Commun., vol. 18, no. 3, pp. 535-547, Mar. 2003.

[178] X. Liu, G. Noubir, R. Sundaram, and S. Tan, "SPREAD: Foiling smart jammers using multi-layer agility," in Proc. 26th Annu. IEEE Int. Conf. Comput. Commun., Anchorage, AK, USA, Mar. 2007, pp. 2536-2540.

[179] Y. Abdallah, M. A. Latif, M. Youssef, A. Sultan, and H. E. Gamal, "Keys through ARQ: Theory and practice," IEEE Trans. 
Inf. Forens. Security, vol. 6, no. 3, pp. 737-751, Sep. 2011.

[180] D. Loh, C. Cho, C. Tan, and R. Lee, "Identifying unique devices through wireless fingerprinting," in Proc. 1st ACM Conf. Wireless Netw. Security, Alexandria, VA, USA, Mar. 2008, pp. 46-55.

[181] V. Brik, S. Banerjee, M. Gruteser, and S. Oh, "Wireless device identification with radiometric signatures," in Proc. 14th ACM Int. Conf. Mobile Comput. Netw., New York, NY, USA, Sep. 2008, pp. 116-127.

[182] W. Hou, X. Wang, J.-Y. Chouinard, and A. Refaey, "Physical layer authentication for mobile systems with time-varying carrier frequency offsets," IEEE Trans. Commun., vol. 62, no. 5, pp. 1658-1667, May 2014.

[183] L. Xiao, L. Greenstein, N. Mandayam, and W. Trappe, "Using the physical layer for wireless authentication in time-variant channels," IEEE Trans. Wireless Commun., vol. 7, no. 7, pp. 2571-2579, Jul. 2008.

[184] Y. Liu and P. Ning, "Enhanced wireless channel authentication using time-synched link signature," in Proc. IEEE Int. Conf. Comput. Commun., Shanghai, China, Mar. 2012, pp. 2636-2640.

[185] J. Xiong and K. Jamieson, "SecureArray: Improving WiFi security with fine-grained physical-layer information," in Proc. 19th ACM Int. Conf. Mobile Comput. Netw., Miami, FL, USA, Sep. 2013, pp. 441-452.

[186] X. Du, D. Shan, K. Zeng, and L. Huie, "Physical layer challenge-response authentication in wireless networks with relay," in Proc. IEEE Int. Conf. Comput.

Commun., Toronto, ON, Canada, Mar. 2014, pp. 1276-1284.

[187] P. Yu, J. Baras, and B. Sadler, "Physical-layer authentication," IEEE Trans. Inf. Forens.Security, vol. 3, no. 1, pp. 38-51, Mar. 2008.

[188] P. Yu and B. Sadler, "MIMO authentication via deliberate fingerprinting at the physical layer," IEEE Trans. Inf. Forens. Security, vol. 6, no. 3, pp. 606-615, Mar. 2011.

[189] P. Yu, G. Verma, and B. Sadler, "Wireless physical layer authentication via fingerprint embedding," IEEE Commun. Mag., vol. 53, no. 6, pp. 48-53, Jun. 2015.

[190] A. Menezes, P. Oorschot, and S. Vanstone, Handbook of Applied Cryptography, Boca Raton, FL, USA: CRC Press, 2001.

[191] M. Latif, A. Sultan, and H. Gamal, "ARQ-based secret key sharing," in Proc IEEE Int. Conf. Commun., Dresden, Germany, Jun. 2009, pp. 1-6.

[192] S. Xiao, W. Gong, and D. Towsley, "Secure wireless communication with dynamic secrets," in Proc. IEEE INFOCOM, San Diego, CA, USA, Mar. 2010, pp. 1-9.

[193] Y. Khiabani and S. Wei, "ARQ-based symmetric key generation over correlated erasure channels," IEEE Trans. Inf. Forens. Security, vol. 8, no. 7, pp. 1152-1161, Jul. 2013.

[194] C. B. Sankaran, "Network access security in next generation 3GPP systems: A tutorial," IEEE Commun. Mag., vol. 47, no. 2, pp. 84-91, Feb. 2009.
[195] Y. Zou, X. Wang, W. Shen, and L. Hanzo, "Security versus reliability analysis for opportunistic relaying," IEEE Trans. Veh. Technol., vol. 63, no. 6, pp. 2653-2661, Jul. 2014.

[196] N. Yang et al., "Safeguarding 5G wireless communication networks using physical layer security," IEEE Commun. Mag., vol. 53, no. 4, pp. 20-27, Apr. 2015

[197] W. Roh et al., "Millimeter-wave beamforming as an enabling technology for 5G cellular communications: Theoretical feasibility and prototype results," IEEE Commun. Mag., vol. 52, no. 2, pp. 106-113, Feb. 2014.

[198] J. Andrews et al., "What will 5G be?" IEEE J. Sel. Areas Commun., vol. 32, no. 6, pp. 1065-1082, Jun. 2014.

[199] C.-X. Wang et al., "Cellular architecture and key technologies for 5G wireless communication networks," IEEE Commun Mag., vol. 52, no. 2, pp. 122-130, Feb. 2014.

[200] P. Rost et al., "Cloud technologies for flexible $5 \mathrm{G}$ radio access networks," IEEE Commun. Mag., vol. 52, no. 5, pp. 68-76, May 2014.

[201] Y. Zou, J. Zhu, L. Yang, Y.-C. Liang, and Y.-D. Yao, "Securing physical-layer communications for cognitive radio networks," IEEE Commun. Mag., vol. 53, no. 9, pp. 48-54, Sep. 2015.

[202] Y. Zou, J. Zhu, X. Li, and L. Hanzo, "Relay selection for wireless communications against eavesdropping: A security-reliability tradeoff perspective," IEEE Network.

\section{ABOUT THE AUTHORS}

Yulong Zou (Senior Member, IEEE) received the B.Eng. degree in information engineering from Nanjing University of Posts and Telecommunications (NUPT), Nanjing, China, in July 2006, the first Ph.D. degree in electrical engineering from the Stevens Institute of Technology, Hoboken, NJ, USA, in May 2012, and the second Ph.D. degree in signal and information processing from NUPT, Nanjing, China, in July 2012.

$\mathrm{He}$ is a Full Professor and Doctoral Supervisor at NUPT. His research interests span a wide range of topics in wireless communications and signal processing, including the cooperative communications, cognitive radio, wireless security, and energy-efficient communications.

Dr. Zou was awarded the 9th IEEE Communications Society AsiaPacific Best Young Researcher in 2014 and is a corecipient of the Best Paper Award at the 80th IEEE Vehicular Technology Conference in 2014. He is currently serving as an editor for the IEEE COMMUNICATIONS SURvers \& Tutorials, IET Communications, and China Communications. In addition, he has acted as a TPC member for various IEEE-sponsored conferences, e.g., ICC/GLOBECOM/WCNC/VTC/ICCC, etc.

Jia Zhu received the B.Eng. degree in computer science and technology from the Hohai University, Nanjing, China, in July 2005 and the Ph.D. degree in signal and information processing from the Nanjing University of Posts and Telecommunications, Nanjing, China, in April 2010.

She is an Associate Professor at the Nanjing University of Posts and Telecommunications (NUPT), Nanjing, China. From June 2010 to June 2012, she was a Postdoctoral Research Fellow at the Stevens Institute of Technology,

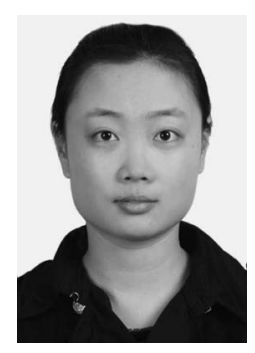

, Hoboken, NJ, USA.
Since November 2012, she has been with the Telecommunication and Information School of NUPT. Her general research interests include cognitive radio, physical-layer security, and communications theory.

Xianbin Wang (Senior Member, IEEE) received the Ph.D. degree in electrical and computer engineering from National University of Singapore, Singapore, in 2001.

$\mathrm{He}$ is a Full Professor at The University of Western Ontario, Lodon, ON, Canada and a Canada Research Chair in Wireless Communications. Prior to joining Western, he was with Communications Research Centre Canada as Research Scientist/Senior Research Scientist be-

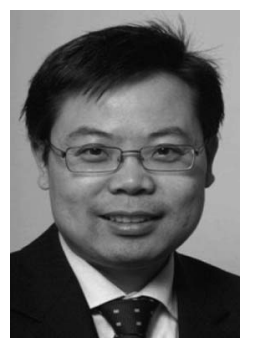
tween July 2002 and December 2007. From January 2001 to July 2002, he was a system designer at STMicroelectronics, where he was responsible for system design for DSL and Gigabit Ethernet chipsets. He was with the Institute for Infocomm Research, Singapore (formerly known as Centre for Wireless Communications), as a Senior R\&D Engineer in 2000. His primary research area is wireless communications and related applications, including adaptive communications, wireless security, and wireless infrastructure-based position location. He has over 150 peer-reviewed journal and conference papers on various communications system design issues, in addition to 23 granted and pending patents and several standard contributions.

Dr. Wang is an IEEE Distinguished Lecturer. He was the recipient of three IEEE Best Paper Awards. He currently serves as an Associate Editor for IEEE WiRELESS COMMUNICATIONS LETTERS, the IEEE TRANSACTIONS ON Vehicular Technology, and the ieEe transactions on Broadcasting. He was also an editor for the IEEE TRANSACTIONS ON WIRELESS COMMUNICATIONS between 2007 and 2011. He was involved in a number of IEEE conferences including GLOBECOM, ICC, WCNC, VTC, and ICME, on different roles, such as symposium chair, track chair, TPC, and session chair. 
Lajos Hanzo (Fellow, IEEE) received the firstclass degree in electronics in 1976 and the Ph.D. degree from the Technical University of Budapest, Hungary, in 1983. He was awarded the Doctor of Sciences (D.Sc.) degree by the University of Southampton, Southampton, U.K., in 2004. In 2009, he was awarded an honorary doctorate by the Technical University of Budapest, and in 2015 by the University of Edinburgh, Scotland.

During his 38-year career in telecommunica-

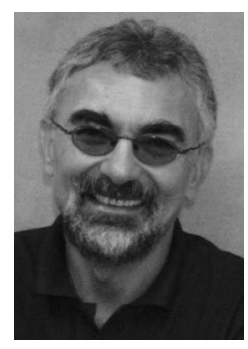

tions, he has held various research and academic posts in Hungary, Germany, and the United Kingdom. Since 1986, he has been with the School of Electronics and Computer Science, University of Southampton, where he holds the Chair in Telecommunications. He has successfully supervised approximately 100 Ph.D. students, coauthored 20 Wiley/IEEE Press books on mobile radio communications totaling in excess of 10000 pages, and published more than 1500 research entries at
IEEE Xplore. Currently he is directing a 60-strong academic research team, working on a range of research projects in the field of wireless multimedia communications sponsored by industry, the Engineering and Physical Sciences Research Council (EPSRC) U.K., the European Research Council's Advanced Fellow Grant and the Royal Society's Wolfson Research Merit Award. He is an enthusiastic supporter of industrial and academic liaison and he offers a range of industrial courses.

Dr. Hanzo is an FIET and a Fellow of EURASIP. He has acted both as TPC and General Chair of IEEE conferences, presented keynote lectures, and has been awarded a number of distinctions. He is also a Governor of the IEEE VTS. During 2008-2012, he was the Editor-in-Chief of the IEEE Press and a Chaired Professor at Tsinghua University, Beijing, China. His research is funded by the European Research Council's Senior Research Fellow Grant. He has more than 23000 citations. 